\title{
Reverse Engineering C++ Software Systems by Detecting Design Patterns
}

\author{
By \\ Peng Xie, B. Eng. \\ A thesis submitted to the Faculty of Graduate Studies and \\ Research in partial fulfillment of the requirements of the \\ degree of
}

\section{Master of Applied Science}

Ottawa-Carleton Institute of Electrical and Computer Engineering Department of Systems and Computer Engineering

Carleton University

Ottawa Ontario, K1S 5B6

Canada

April 2003 


$\begin{array}{ll}\begin{array}{l}\text { National Library } \\ \text { of Canada }\end{array} & \begin{array}{l}\text { Bibliothèque nationale } \\ \text { du Canada }\end{array} \\ \begin{array}{l}\text { Acquisitions and } \\ \text { Bibliographic Services }\end{array} & \begin{array}{l}\text { Acquisisitons et } \\ \text { services bibliographiques }\end{array} \\ \begin{array}{l}\text { 395 Wellington Street } \\ \text { Ottawa ON K1A ON4 }\end{array} & \begin{array}{l}\text { 395, rue Wellington } \\ \text { Ottawa ON K1A ON4 } \\ \text { Canada }\end{array} \\ \end{array}$

Your file Votre référence ISBN: 0-612-83484-0 Our file Notre référence ISBN: 0-612-83484-0

The author has granted a nonexclusive licence allowing the National Library of Canada to reproduce, loan, distribute or sell copies of this thesis in microform, paper or electronic formats.

The author retains ownership of the copyright in this thesis. Neither the thesis nor substantial extracts from it may be printed or otherwise reproduced without the author's permission.
L'auteur a accordé une licence non exclusive permettant à la Bibliothèque nationale du Canada de reproduire, prêter, distribuer ou vendre des copies de cette thèse sous la forme de microfiche/film, de reproduction sur papier ou sur format électronique.

L'auteur conserve la propriété du droit d'auteur qui protège cette thèse. $\mathrm{Ni}$ la thèse ni des extraits substantiels de celle-ci ne doivent être imprimés ou aturement reproduits sans son autorisation. 
The undersigned recommend to the Faculty of Graduate Studies and Research Acceptance of the thesis:

\section{Reverse Engineering C++ Software Systems by Detecting Design Patterns}

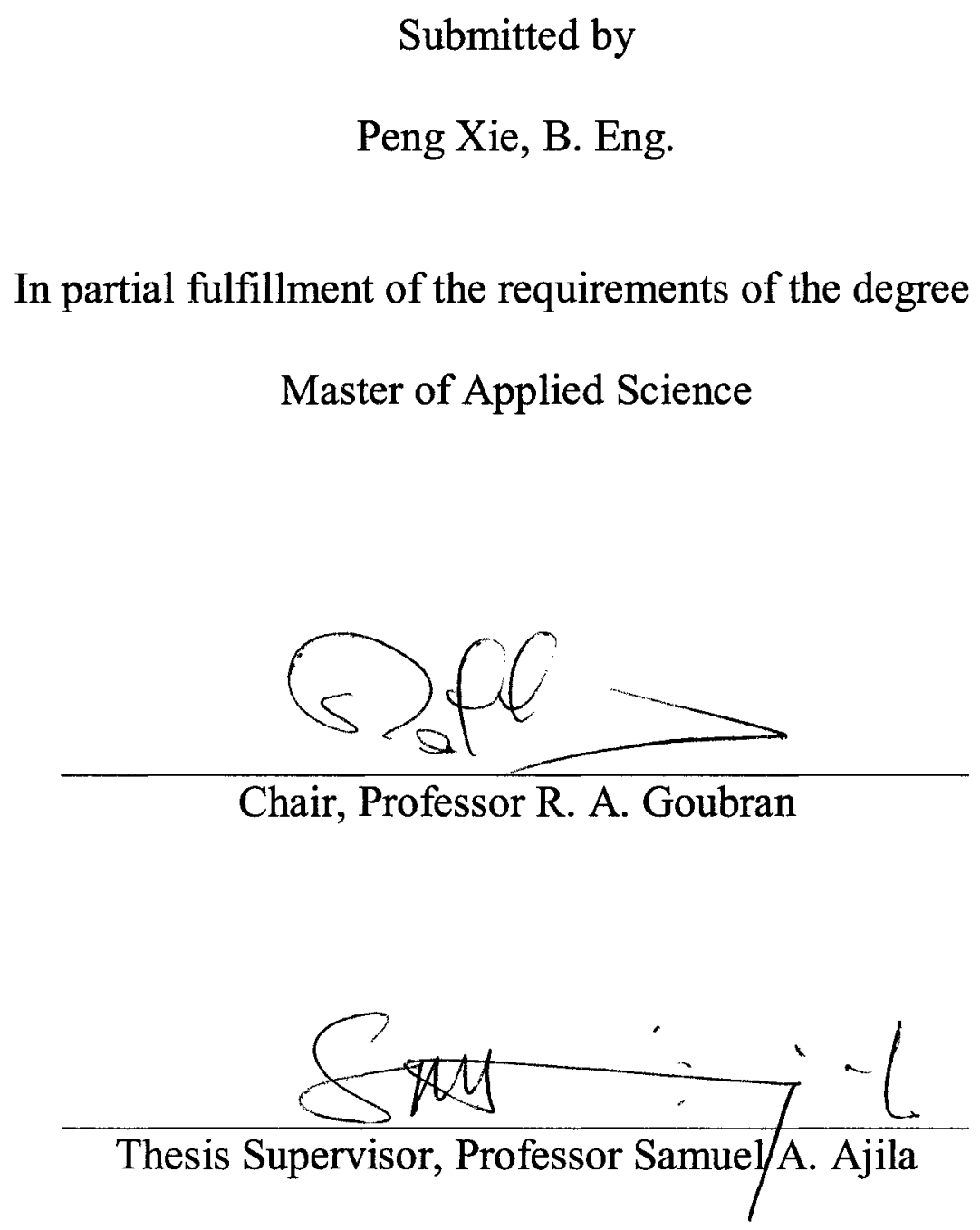

Carleton University

May 2003 


\section{Abstract}

Reverse engineering plays an important role in the comprehension and evolution of software systems. Detecting design patterns in software systems is the first major step toward understanding software systems at a pattern level in order to gain insight into their structure and behavior.

This thesis proposes a rule-based approach to detecting design patterns in software systems. A model for the formal description of design patterns is proposed based on both static and dynamic characteristics of design patterns. It is represented as logic rules in a rule base, while the design information extracted from source code is represented as logic facts in a fact base. The fact and rule base are integrated as a knowledge base. By querying this knowledge base, we can detect patterns enclosed in source code. The features of logic programming make the implementation of the approach simple, flexible and extensible.

Two case studies have been carried out in this thesis. The results and their comparisons indicate the effectiveness and improvement of our approach in this area.

As design patterns have been widely used in software development, a patternlevel view of software systems will be more helpful for software maintainers and developers. 


\section{Acknowledgments}

I would like to express my incredible gratitude to my supervisor, Dr. Samuel A. Ajila for his patient guidance and continued encouragement. Without his great help, I wouldn't have completed this research successfully.

The financial assistance provided by Carleton University was greatly appreciated. Finally, special thanks to my parents and my wife whose support has helped me strive to attain my goals in all of my endeavors. 


\section{CONTENTS}

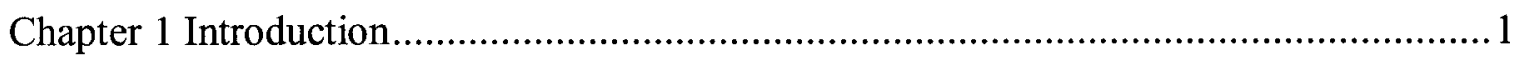

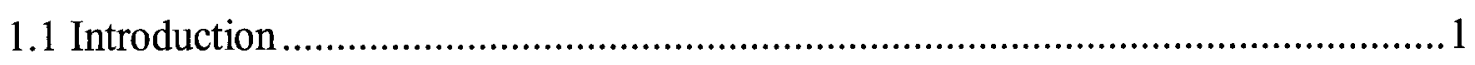

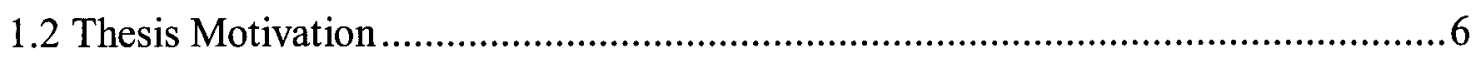

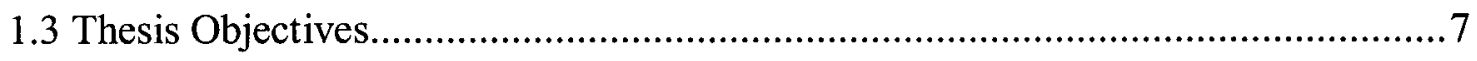

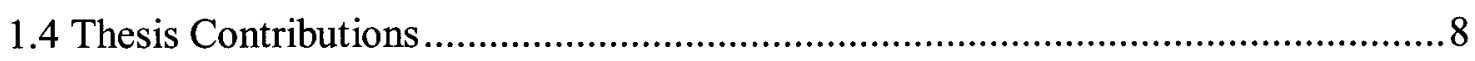

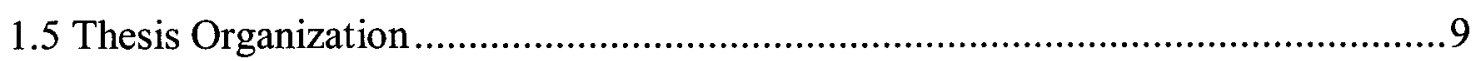

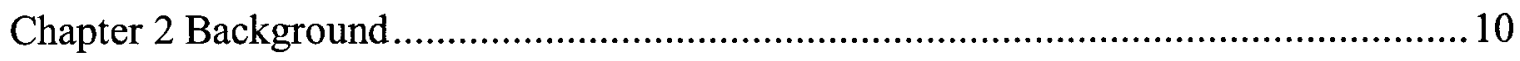

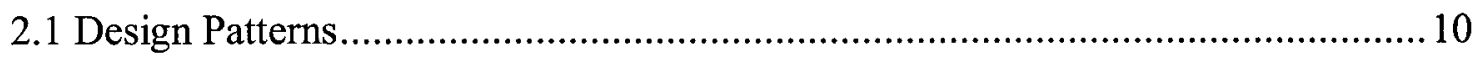

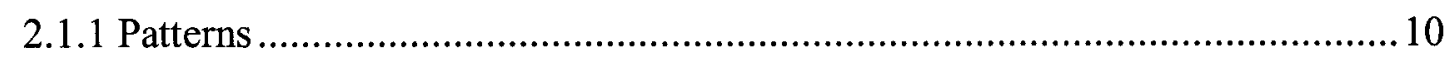

2.1.2 Classification of Software Patterns ............................................................ 12

2.1.3 Design Patterns in Design Level................................................................13

2.2 Design Patterns in This Thesis ........................................................................ 13

2.2.1 The GOF Patterns and Catalog …………………..................................... 14

2.2.2 General Pattern Description .................................................................... 14

2.2.3 Usage of the Catalog and the Patterns.......................................................... 15

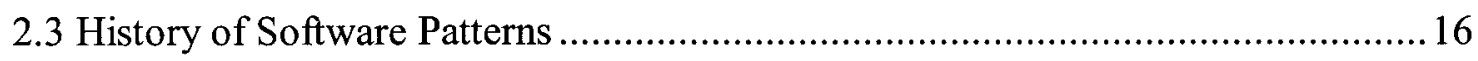

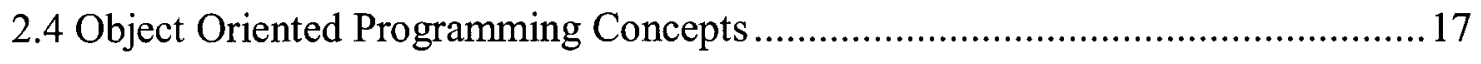

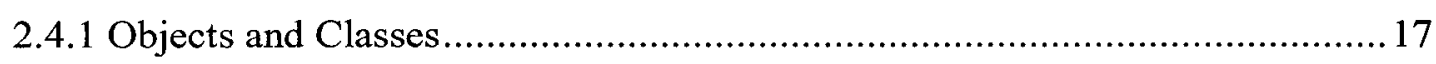

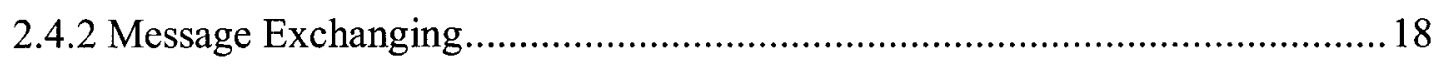

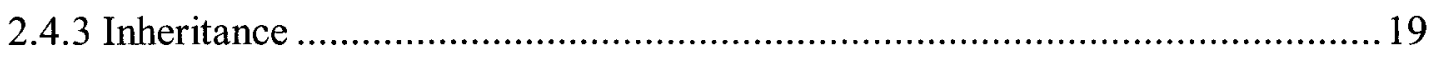

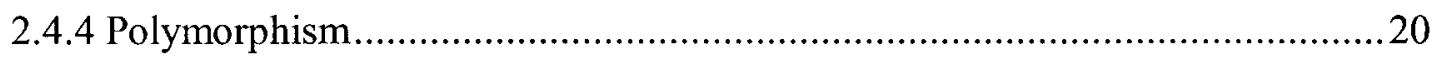




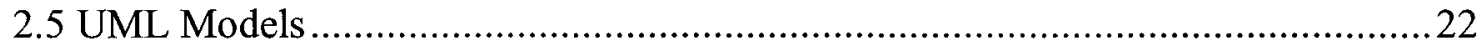

Chapter 3 Related Works and Thesis Problem Statement ..........................................225

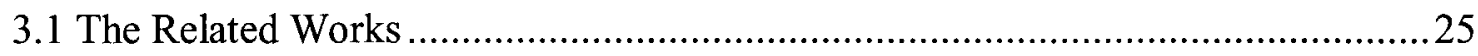

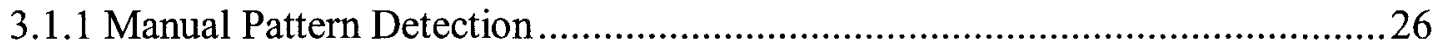

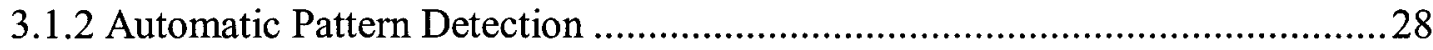

3.2 Problem Definition in This Thesis ............................................................ 32

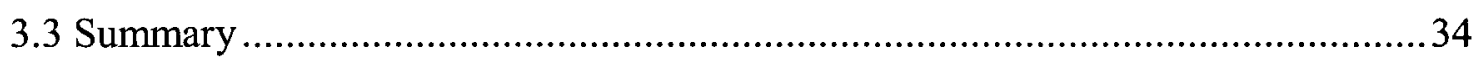

Chapter 4 An Approach to Design Pattern Detection ............................................ 35

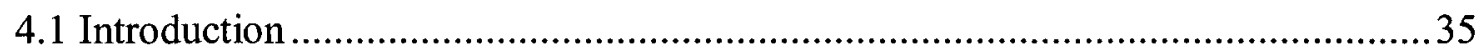

4.2 Formal Description of Components and Relationships ................................... 37

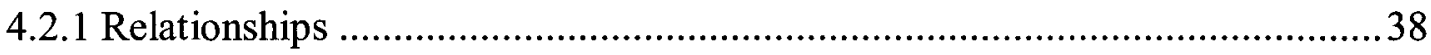

4.3 Prolog Representation of the Formal Description........................................47

4.3.1 Components ............................................................................ 47

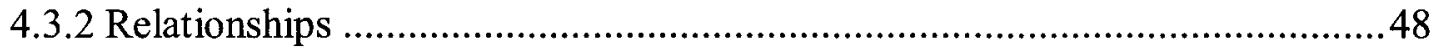

4.4 Formal Description of Design Patterns........................................................ 51

4.4.1 The Models of Creational Design Patterns............................................5 52

4.4.2 The Models of Structural Design Patterns ............................................... 54

4.4.3 The Models of Behavioral Design Patterns...............................................6 65

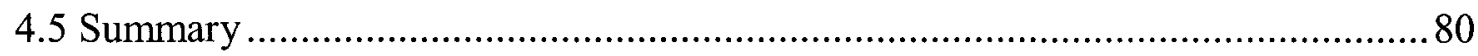

Chapter 5 Design Pattern Extraction Tool (DPET) ............................................ 81

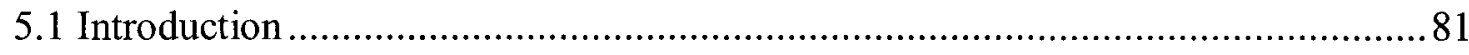

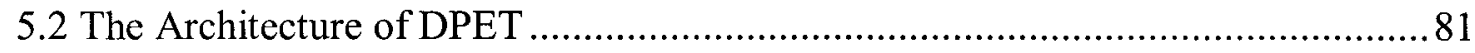

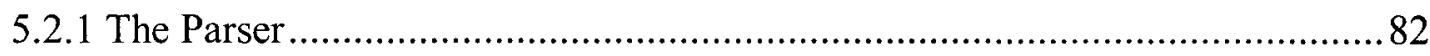




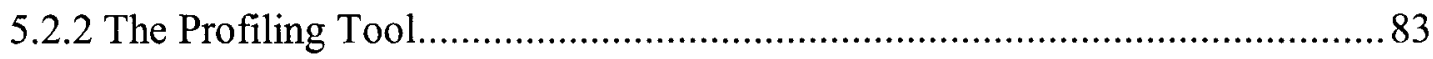

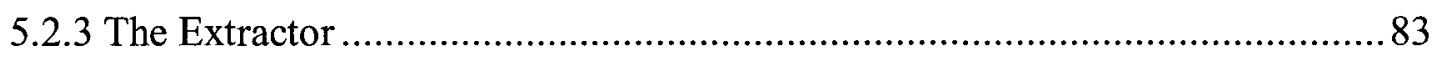

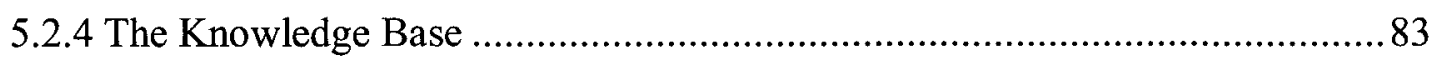

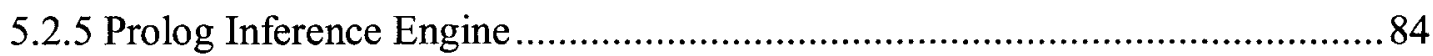

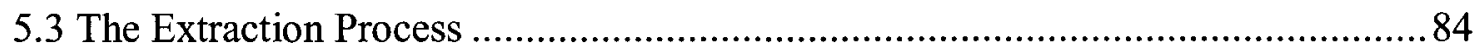

5.3.1 Extraction Description of Proxy Design Patterns ......................................... 84

5.3.2 Extraction Description of Adapter Design Patterns......................................... 89

5.3.3 Extraction Description of Dynamic Information using Profiling .....................93

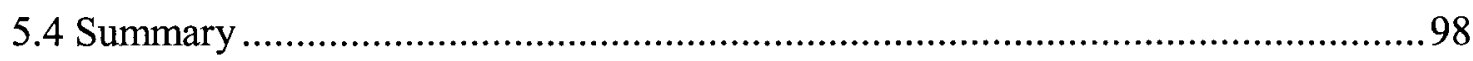

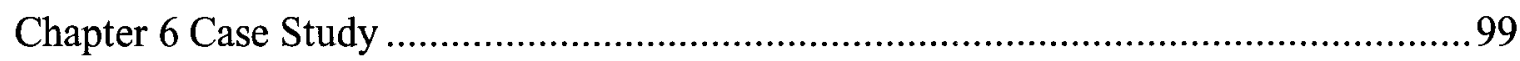

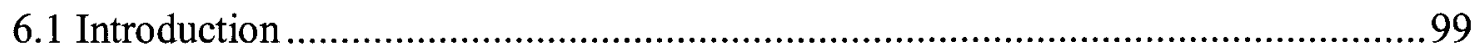

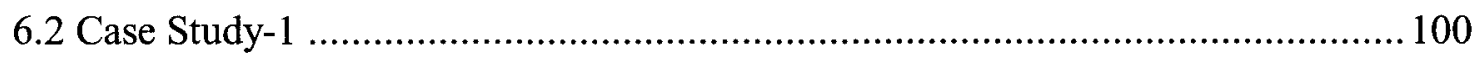

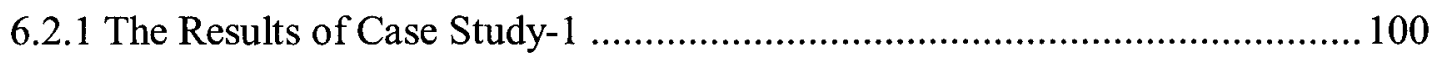

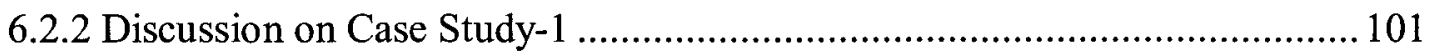

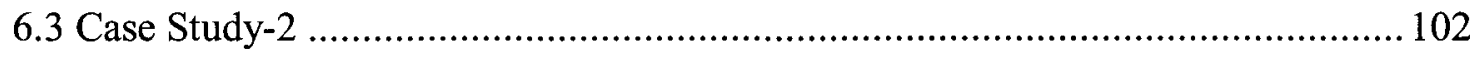

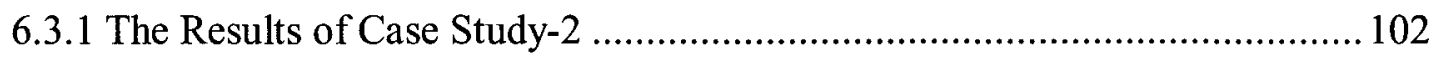

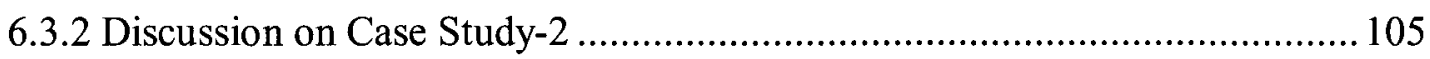

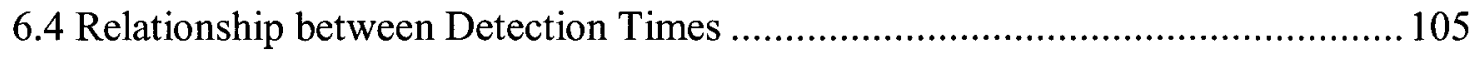

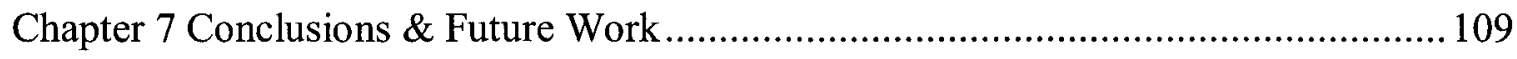

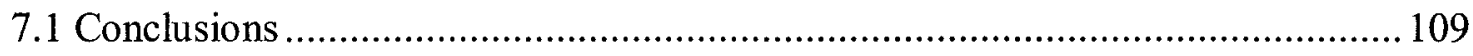

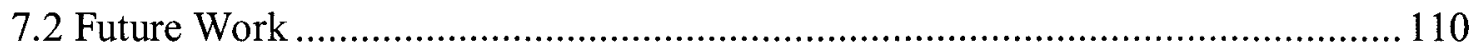

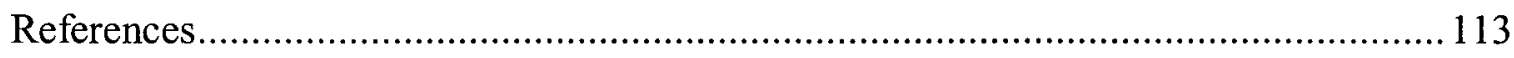

Appendix A The Detailed Extraction Process of Proxy and Adapter Patterns................ 120 
A.1 Extraction Description of Proxy design patterns

A.2 Extraction description for Adapter design patterns 125

Appendix B The Data Format for Source Code Information

Appendix C The Results Of Profiling The Sample Code of Chain of Responsibility

Design Patterns

Appendix D Class Diagrams in LQNS

D.1 Class hierarchy of Server class in LQNS. 143

D.2 Part of Class Diagram of LQNS 144 


\section{List of Figures}

Figure 1-1 Relationship between forward engineering and reverse engineering in terms of life cycle phases [Chi90]..................................................................................

Figure 4-1 UML class diagram of Delegation relationship ...........................................44

Figure 4-2 UML class diagram of Factory Method design patterns ...............................52

Figure 4-3 UML class diagram of Adapter design patterns ..........................................54

Figure 4-4 UML class diagram of Composite design patterns ........................................56

Figure 4-5 UML class Diagram of Decorator design patterns ........................................59

Figure 4-6 UML class diagram of Proxy design patterns...............................................62

Figure 4-7 UML class diagram of Bridge design patterns .............................................64

Figure 4-8 UML class diagram of Strategy design patterns ............................................66

Figure 4-9 UML class diagram of Observer design patterns .........................................68

Figure 4-10 UML sequence diagram of Observer design patterns ..................................71

Figure 4-11 UML sequence diagram of Chain of Responsibility design patterns .............74

Figure 4-12 UML class diagram of Visitor design patterns ..........................................76

Figure 5-1 The architecture of the tool DPET ………................................................. 81

Figure 5-3 UML representation of the "Adapter" design pattern .....................................93 


\section{List of Tables}

Table 4.1 The Composition of the model for formal description of design patterns ........38

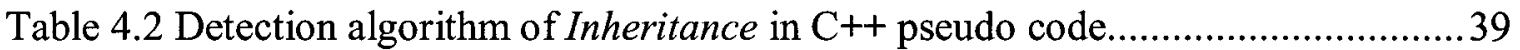

Table 4.3 Detection algorithm of Aggregation in C++ pseudo code. ..........................40

Table 4.4 Detection algorithm of $U$ sing relationship in $\mathrm{C}++$ pseudo code ....................42

Table 4.5 Detection algorithm of Association in pseudo code. ....................................43

Table 4.6 Detecting Delegation algorithm in $\mathrm{C}++$ pseudo code. .............................44

Table 4.7 Detecting CreationLink algorithm in C++ pseudo code .............................46

Table 4.8 Detecting SendMessage algorithm in pseudo code .................................46

Table 4.9 Detecting SelfMessage algorithm in C++ pseudo code .............................47

Table 6.1 LQNS code characteristics and detection time................................. 100

Table 6.2 Results of pattern detection in LQNS using our tool DPET ....................... 101

Table 6.3 Code characteristics of three pieces of software .................................. 102

Table 6.4 Results of pattern instance detection using our tool DPET .................... 103

Table 6.5 Results of pattern detection using the approach proposed by Antoniol.......... 104

Table 6.6 Comparison of pattern instances obtained by DPET and MsA................... 104

Table 6.7 Code characteristics and detection time of each software system................ 106

Table 6.8 Detection time table after not detecting some relationships ....................... 107

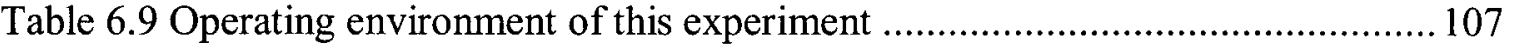




\section{Chapter 1 Introduction}

\subsection{Introduction}

Reverse engineering (cf. figure 1-1) is "the process of analyzing a software system to identify the system's components and their interrelationships and create representations of the system in another form at a higher level of abstraction" [Chi90]. It is a process of examination, rather than change or replication. The aim of reverse engineering is to provide an overview of legacy software systems. It is the first major step towards understanding the evolution of a software system. Reverse engineering is a necessary component of software maintenance in that it enables us to understand the information embedded in the code.

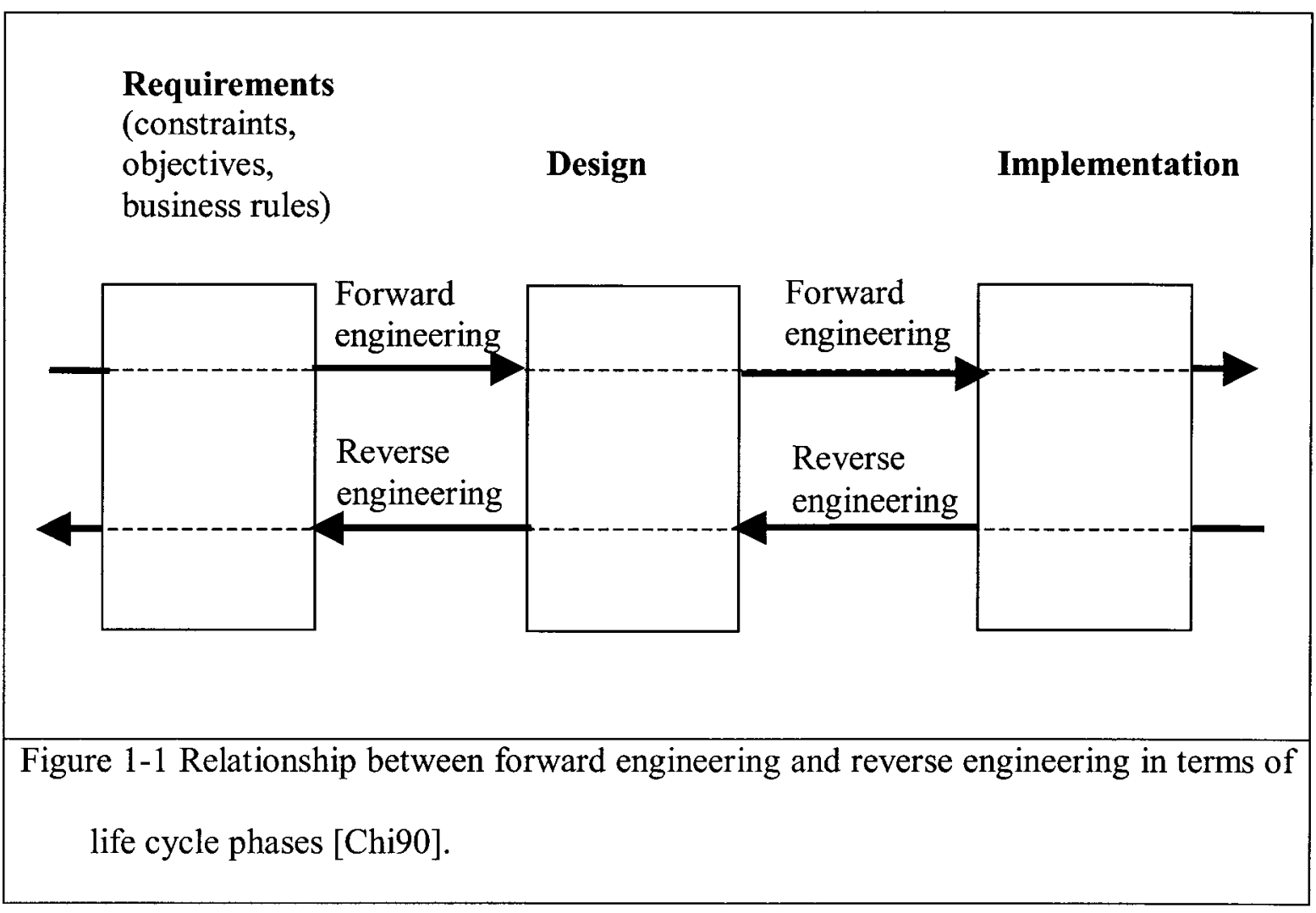


The task of reverse engineering is to identify and recover requirements and design specifications. This high-level design information helps in understanding a software system. In general, such high-level information extracted from source code includes [Lu00]:

- System structure- its components and their interrelationships, as expressed by their interfaces

- Functionality - which operations are performed by which components

- Dynamic behavior - a systematic understanding about how input is transformed to output

- Rationale - design involves decision making between a number of alternatives at each design step in the design

- Construction - modules, documentation, test suites, etc.

Reverse engineering plays an important role in software maintenance; it is the foundation of re-engineering. Re-engineering is the examination and alteration of a software system to reconstitute it in a new form and the subsequent implementation of the new form [Chi90]. Re-engineering (cf. Figure 1-1) includes some form of reverse engineering (to achieve a more abstraction description) followed by some form of forward engineering or restructuring.

The following are some of the research techniques [Hon01, Sys00, Rea00, Ros99, Mu198, Cha98, Jer97, Che97, Ga96, Gan96, Bus96, My194, Che94, Wi193, Ben92, Har90 Raj90] applied in reverse engineering [Lu00]:

- Program understanding - The objective of program understanding is to extract information useful for comprehension from source code and represent it in such a 
way that conforms to human cognitive habits. Program understanding can be conducted in a formal or an ad hoc manner.

- Specification recovery - Specification recovery is a bottom-up process in which a description of the functional and nonfunctional requirements of a software system is extracted from source code.

- Design recovery- Design recovery is a process whereby design information is recovered from the code. Its objective is to extract design information from source code. There are two strategies to achieve this.

- Fundamental method. The source code is represented in such a way that a maintainer can conduct abstraction. In this method, the computer retrieves information that is entirely embedded in the source code.

- Knowledge-based method. In this method the tool's aim is to make the abstraction automatically or semi-automatically. The source code is anaiyzed by using information from a knowledge base.

The most popular approaches in design recovery are as follows:

1. Code views - A part of or the entire source code is represented in a certain way to speed the comprehension of software systems. The information included in the representations is the same as the source code. E.g., call graphs, data flow, definition-use graphs and/or control dependencies.

2. Reformatting and Markup Languages - Reformatting changes the structure of the source code without changing its functions in order to improve readability. Markup languages are languages for annotations of 
source code simply to improve the source code's appearance by the means of bold-faced key words, slanted comments, etc.

3. Hypertext-This is used to help browse the source code.

4. Source code analysis with program transformation and abstraction rulesSource code is analyzed using the concrete rules of program transformation and abstraction; these are defined according to concrete source code language. A sophisticated abstraction can be obtained from source code by searching for certain types of knowledge in a database using these rules.

5. Data-Centered and control-centered program understanding - Datacentered methods focus on data and data relationships whereas controlentered methods focus on the control structure of a program, such as call graphs, control flow graphs and paths.

5. Program slicing - a large program is decomposed into some functional self-contained slices (program fragments) in which only the statements useful for understanding the program are kept.

- Architecture recovery - Architecture gives a global picture of the whole software system. Architecture recovery aims to extract the system architecture from the legacy source code and available documentation.

- Business rules extraction - The business rules for an organization are extracted from legacy software systems.

- Cognitive processes in human program understanding- this research issue focuses on the process in which a human reader understands the source code, through 
cooperating with computer technology. The study of human cognitive processes can improve reverse engineering by making the approach and tools more supportive to human understanding.

- Intermediate representation of source code - Source code is preprocessed and is represented as an intermediate representation that stores the same information as the source code. Querying this intermediate representation to get high-level view of a software system will be faster than querying the source code directly. It can be used to improve the efficiency of the re-engineering of large systems. In this research direction there are two main topics as follows:

- Data and knowledge-based representation

The source code information is stored in data and a knowledge-based representation so that the design information can be extracted by querying the knowledge base. Relevant artificial intelligence technologies are used to facilitate and optimize the process of reverse engineering.

- Graph based representation

The source code information is represented in a certain form of graph to provide a more perceptive overview, e.g., ASG (Abstract Syntax Graph).

- Discovery of reusable components - This technique focuses on identifying and extracting existing reusable components from legacy software systems.

- Knowledge-based analysis - knowledge-based analysis in reverse engineering enables re-engineering tools to become so smart that frequently used domain knowledge can be recovered automatically. 
The subject of this thesis belongs to the 'design recovery' group. The aim is to detect design patterns in software systems. The model presented in this thesis is a rule-based approach.

\subsection{Thesis Motivation}

During the development process, software systems often have incomplete, incorrect or even nonexistent design documentation due to short time to market, staff mobility and costs. After their delivery some software systems have evolved due to requirement change, error correction, business rule changes, and structural reorganization. In these cases, the original design documentation may not always reflect the updated information contained in the current code. Therefore the source code is the only highly reliable source for obtaining the precise design information.

In general, UML class diagrams could be used to represent design information extracted from source code. A class diagram can show the classes and their interrelationships that are included in source code. This is really helpful for understanding the software system. However, as the software systems grow larger it is very hard to get a high-level view of software systems from the several hundreds of classes and their interrelationships.

A higher-level abstraction is needed to simplify the complexity of class diagrams.

One such high-level abstraction is the design pattern. Design patterns can be viewed as micro-architectures for solving particular design problems because they reflect the structure and behavior of a set of classes in a software system. A design pattern records the experts' experience in solving a recurring problem using a general way so that 
software developers can easily make a good design and make software development more efficient and time-saving.

Looking for patterns in the source code makes it easier to fully understand the way the software system is structured. A design pattern specifies the role of each class in the pattern, the relationships among the classes and interactions among instances of classes.

By using design patterns when extracting design information we can understand a piece of code at pattern level, a higher abstraction level than the design and implementation level, but lower than the architecture level.

Therefore, design patterns provide good insight into the structure and behavior of a software system.

\subsection{Thesis Objectives}

This research is motivated by the need for a high-level abstraction to simplify the complexity of software systems for better comprehension within reverse enginecring. Design patterns represent a high-level abstraction. In order to acquire such a high-level abstraction from the source code, we need an approach to effectively identify design patterns from the design information extracted frum the source code.

The objectives of this thesis are summarized as follows:

- To examine the entire source code for complete program information.

- To define a model for a formal description of design patterns.

- To propose an approach to effectively identify design patterns according to this model.

- To apply this approach to some case studies and validate it. 
- To analyze the results obtained from the case studies.

\subsection{Thesis Contributions}

This thesis proposes a simple model and tool for the extraction of design patterns from $\mathrm{C}++$ code.

1. The model follows a rule-based approach. This approach exploits two major concepts of logic programming:

- Flexibility - comes from the underlying principle of logic programming, that is, it is possible to change the rule base without affecting the inference engine acting on the rules.

- Declarative nature of programming in logic-like languages (e.g. Prolog)this helps to avoid the specification of a lot of execution control constraints.

2. The model examines both static and dynamic information from the source code. In this case, the tool will only work on a code that is compliable and executable

3. A formal approach is developed for the detection of relationships among software components. The set of algorithms from this approach is used to extract design information.

4. The extraction of design information is separated from design pattern recovery in order to facilitate flexibility. This way, the design pattern recovery is languageindependent while the information extraction is language-dependent. The positive thing here is that the tool can be extended to capture other programming languages (e.g. Java) without significantly affecting the design of the design pattern recovery section. In addition a user of the tool could decide to introduce 
new rules into the rule base while keeping the fact base intact, this can happen as long as the relationships detection algorithms syntax is respected.

5. The parser that is developed for the static analysis captures the entire source code. In our opinion, this is better than just capturing the header files alone. The header files do not represent the complete information in the code.

6. A prototype tool DPET (Design Pattern Extraction Tool) is implemented by the rule-based approach proposed in this thesis.

7. In addition, the intermediate results (i.e. fact base) could be used to visualize the class diagram representing the static information of the system under review (cf. Appendix D)

\subsection{Thesis Organization}

The rest of this thesis is structured as follows:

Chapter 2 provides background information about software design patterns. It describes briefly the concepts of pattern, design pattern, object-oriented program and UML.

Chapter 3 states the problem to be solved in this thesis followed by a review of the state of the art of related work in this research direction. Chapter 4 proposes a model to formalize design patterns in Prolog presentation in terms of static and dynamic characteristics of design patterns. It describes a set of detection algorithms based on design information and pattern detection algorithms. Chapter 5 describes a prototype tool DPET implemented according to the model proposed in Chapter 4, the architecture of the tool and the process of extraction. Two case studies are provided in Chapter 6 to validate our approach. Chapter 7 summarizes the conclusions and points to the direction future research should be heading. 


\section{Chapter 2 Background}

This chapter provides background information related to this thesis. The concepts from design patterns to object-oriented programming are presented briefly, in order to make the thesis self-contained.

\subsection{Design Patterns}

\subsubsection{Patterns}

From kids to adults we all have learned a lot about how we do things from our parents, teachers and friends, etc. If we want to do many things, we know how to do it according to schemes. For instance, if you want to drive a car, you have to operate it according to some schemes or rules. Otherwise if you don't you may have a bad experience in driving, or even maybe unable to starr the car. After several years of driving you may become an expert and the scheme of driving a car have become a mental pattern. This mental pattern has been engraved firmly in your mind, later on you don't have to think of how to drive a car, it becomes reactionary, and you can even teach others to drive. You have learned to do it according to both your own experience and others' experience. Such schemes of how to do things are introduced in the software engineering and are called software patterns.

The simplest definition of a pattern is "a solution to a problem in a context"[Gam95]. The mental pattern in the above example is just a solution to the problem of how to drive a car in the context of safe driving. The term "pattern" adopted in software engineering comes from the work of Christopher Alexander, an architect who was exploring patterns in architecture. Through his work he recorded design principles in 
architecture in order to prevent future architects from making mistakes. He categorized architectural design patterns so that other architects could acquire his knowledge. The definition of a pattern given by Alexander is as follows: "Each pattern describes a problem which occurs over and over again in our environment, and then describes the core of the solution to that problem, in such a way that you can use this solution a million times over, without ever doing it the same way twice [Ale79]."

A pattern can be viewed as a prose format for recording solutions that have worked well in the past and can be applied again in similar situations in the future [Bec96]. The need for the introduction of patterns in software originates from the need of the engineering discipline to mature, and to provide guidelines for describing successful solutions to known problemis.

A pattern is a way to provide information on the form of a problem statement, constraints on the problem and presentation of a widely accepted solution to the problein and then a discussion of the consequences of that solution. Patterns are expressed textually in an informal manner, their description of a name, a problem and its associated context, a discussion and a solution. A description of a design pattern includes not only the static structure based on classes and objects involved in the pattern and their relationships, but also the behavioral pattern dynamics based on the exchanged messages. In order to be considered a pattern the solution must be verified as a recurring solution to a recurring problem. 


\subsubsection{Classification of Software Pattems}

In general the software community focuses mainly on design patterns, which are described by [Bec96, Gam95, Pre94]. However, patterns can be found in all aspects of software such as development organization, software process, project planning, requirements engineering, and software configuration. There are many different classifications of software patterns; one possible classification of patterns is as follows [App00]:

- Design patterns: the patterns in software engineering.

- Analysis patterns: the patterns that describe recurring and reusable analysis models.

- Organization patterns: the patterns that describe software process design.

- Other domain-specific patterns.

Reihle and Zullighoven [Rei96] propose a different classification of patterns; the three types of patterns they define are as follows:

- Conceptual patterns, which are described by rneans of the terms and concepts from an application domain, which are geared towards a restricted application domain.

- Design patterns, which are described by means of software design constructs, e.g., objects, classes, inheritance, aggregation, and reuse relationship. Design patterns complement the conceptual space opened by the conceptual patterns.

- Programming patterns, which are described by means of programming language constructs. 


\subsubsection{Design Patterns in Design Level}

Design patterns can be divided further into three categories of patterns based on the level of abstractions [Bec96]:

- Architectural patterns, Are high-level strategies that are concerned with largescale components and global properties and mechanism of a system. Architectural patterns have implications affecting the overall structure and organization of a software system.

- Design patterns, Are medium-level strategies that are concerned with the structure and behavior of components, and their relationships. Design patterns do not influence the overall system structure, but instead define micro-architectures of subsystems and components. Design patterns in this classification correspond to design patterns in the classification by [Rei96], and to GOF patterns, i.e., patterns described by [Gam95].

- Idioms, are paradigm-specific and language-specific programming techniques that fill in low-level internal and external details of a component's structure or behavior. Jim O. Coplien describes idioms in $\mathrm{C}++$ [Cop92].

The difference between these three categories of design patterns is their corresponding levels of abstraction and detail.

\subsection{Design Patterns in This Thesis}

This thesis is based on medium-level software design patterns as mentioned above, that is, the GOF design patterns categorized in [Gam95]. The GOF design patterns 
concerned with the structure and behavior of components and their interrelationships.

Design patterns have no impact on the whole system structure, but define microarchitectures of subsystems and components. They specifically deal with common problems in OO design [Gam95], which are the problems that are often encountered again and again in OO design. Design patterns provide general solutions to these common problems.

\subsubsection{The GOF Patterns and Catalog}

The catalog mentioned in [Gam95] consists of 23 design patterns that are called GOF (Gang Of Four) patterns and introduces an informal way to describe them. According to the terminology proposed by [Gam95], the design patterns can be classified into creational, structural and behavioral depending on their purpose and scope. Creationd patterns concern object creation, where as structural patterns capture class or object composition. Behavioral patterns deal with the way in which classes and objects distribute responsibility andi interact.

\subsubsection{General Pattern Description}

The GOF patterns are described in a consistent form and in natural language. The general form is as follows:

- Pattern name: The short name to refer to the pattern and the problem it solves.

- Intent: Brief description of what issue the pattern addresses.

- Also known as: Other names for the pattern, if there are any.

- Motivation: An example design problem that is solved with the pattern at hand

- Applicability: the context in which the pattern can be applied. 
- Structure: A graphical scheme of the pattern, usually shown by a UML class diagram.

- Participants: Description of the sets of participants in the pattern and their responsibilities.

- Collaborations: The way in which the participants interact to solve the problem.

- Consequences: The results of applying this pattern, and the trade-offs.

- Implementation: how to implement this pattern.

- Sample Code: Sample code fragments of implementing the pattern in OO language.

- Known Uses: Good examples of real software systems that used the pattern.

- Related Patterns: The patterns that are closely related to this pattern.

\subsubsection{Usage of the Catalog and the Patterns}

Design patterns originally have been used in software development. When we design object-oriented software, we want to make a solid design so as to reuse it for other software later on. When looking for patterns in the catalog for a new design you will have to decide what pattern to apply. First of all you should have a thorough knowledge of the problem you are trying to solve. Then, you should know what kind of patterns in the pattern family could be applied to deal with it. In order to do that, checking the general description of design patterns is the first step towards an application of the patterns. The Intent section indicates what that pattern does. The Motivation and Applicability part of the pattern description indicates what the meaning of the pattern is. When you cannot find the information you want, the Related Patterns section might give some hints for other choices. 


\subsection{History of Software Patterns}

The concept of "pattern" originates from the idea of Christopher Alexander, an architect, who wrote several books about patterns in urban planning and building architecture were published between 1964 and 1979. The most famous being is The Timeless Way of Building [Ale79].

In 1987 Ward Cummingham and Kent Beck first used some of Alexander's ideas to develop a small pattern language for guiding novice Smalltalk programmers. The result of their work was presented in the paper Using Pattern Language for ObjectOriented Programs at OOPSLA in 1987.

In the following years, Jim Coplien began to study language-specific $\mathrm{C}++$ patterns so-called idıoms. The book Advanced $C++$ Programming Styles and Idioms [Cop92] was published in 1992.

In 1990 members of GOF started work on compiling the catalog of patterns. At the OOPSLA workshop given by Bruce Andersen, in 1991, great progress was achieved since many of the pattern notables took part in the workshop, e.g., Jim Coplien, Doug. Lea, Desmond D'Souza, Norm Kenth, Wolfgang Pree, and members of the GOF.

In August of 1993, Kent Beck and Grady Booch sponsored a mountain retreat to Colorado, the first meeting of what is now called the Hillside Group. Another pattern workshop was held at OOPLA in 1993, and in 1994, shortly after the book by GOF, Design patterns: Elements of Reusable Objects [Gam95], was published. The book is a milestone in software engineering and has had an enormous impact on software development. 


\subsection{Object Oriented Programming Concepts}

The term "Object Oriented" means that software is organized as a collection of discrete objects that incorporate both data structure and behavior [Rum91]. Object oriented programming originates as a discipline for imperative programming. With imperative programs growing bigger, the conventional programming is getting more unmanageable because there are some unavoidable drawbacks. E.g., a global variable can be accessed and updated anywhere and at anytime in a program.

An object is a software bundle of related variables and operations. Software objects are often used to model real-world objects you find in everyday life. An object model captures the static structure of a system by showing the objects in the system, relationships between objects, and the attributes and operations that characterize each class of objects.

Objects are instances of a particular class and classes are organized in a hierarchy of subclasses and super-classes; an object will inherit the attributes and behaviors of all its super-classes.

\subsubsection{Objects and Classes}

A class describes the underlying structure of a group of objects. It could be said that a class is a blueprint, and an object is a house. An object belonging to a class is also called an instance of that class.

A class specifies the data items of each object and contains the operations or methods that can be performed on each object within that class (in object-oriented languages that lack generic functions). Collaborating objects compose an object-oriented 
program, in which each object has a specific behavior and state; this behavior may depend on the state of the object. The class can be compared to a mold for producing specific objects. The things that an object in that class can do are called operations. Operations, like functions, live inside an object. Some operations that can be invoked by other objects are called public operations, while other operations that can be used only by the object itself are referred to as private operations.

Object-oriented languages create new objects in one of two ways; some languages, like Smalltalk, have classes that are full objects in their own right. In these languages, an operation applied to a class object (called a class method) creates a new object of the class. Other languages, such as $\mathrm{C}++$ and Eiffel, do not have class objects. These languages have special operations that are responsible for creating new objects.

There are some classes without instances and they just capture common behavior for consistency of the class hierarchy and reuse. They are called abstract classes as opposed to concrete classes of which instances can be instantiated.

\subsubsection{Message Exchanging}

As mentioned above, object-oriented programs consist of objects. There must be some way to communicate among objects; operations are responsible for it. By sending each other messages, objects invokes an operation of another object, which is called SendMessage in this thesis, or its own operation, which is called SelfMessage in this thesis. The message might be a call for information, or a question like changing the state of objects. In order to do so, an object has to know about other objects to which messages are sent. An object "knows" another object when one is the instance variable of another, or one can create another by sending a construction message ("new" in C++ term) to the 
latter's class. Depending on a possible result of a message send, the object to which the message is sent might change its state or send more requests to other objects.

\subsubsection{Inheritance}

Car and aircraft are both part of a bigger community called MobileVehicle. Both are used as vehicles that can load persons or things and move from one place to another place. However both are different in many specific aspects, e.g., a car moves on roads . while an aircraft flies in the sky. The class MobileVehicle can be used as a superclass that captures common behavior among families of both objects; cars and aircrafts can be used as subclasses to capture their own specific behavior. In this way, the classes of Cars and Aircrafts are subclasses of MobileVehicle, and both inherit the behavior of MobileVehicle. In some object. oriented languages, classes can have multiple superclasses from which behavior is inherited. This is called multiple-inheritance.

In order to provide a difference in behavior for the same request, subclasses might define a new operation with the same name instead of using the inherited one. We call this overriding.

In case the overriding procedure needs the original operation to do its job, the original operation can be called upon by sending a message to a superclass.

In typed object-oriented language classes there may be overloaded operations. These are operations with the same name, but they differ in the type of their arguments. This concept is called overloading. 


\subsubsection{Polymorphism}

Polymorphism means "many forms." In object-oriented programming it enables one operation to operate differently on different types of objects, providing a useful abstraction that enables users of that operation to treat dissimilar objects in a similar manner.

There may be times when you want to treat different objects in similar ways. For example, a Car class might be passed to an operation named move, which is responsible for moving the car in a certain direction at a certain speed. An aircraft class also has move operation, but both don't move in the same way. For example, an aircraft is propelled through the air by propellers installed on the wings of the aircrafts. On the other hand, cars move in a very different manner from aircraft. Their wheels "push" them by creating directional friction against the road. Internally, the processes are very different in propelling each vehicle.

With object-oriented programming, being able to create abstractions that hide such differences is part of what makes them so powerful. With an abstraction such as the move operation, you could make an aircraft move the same way that you could make a car move, without having to worry about the details that are "hidden" beneath the abstraction. The command move (car1, 100, "east") would make a truck go 100 $\mathrm{Km}$ per hour in an eastbound direction just as move (aircraft1, 100, "east") would make an aircraft move in the same direction at the same speed. Consider the example shown in the following code listing. 


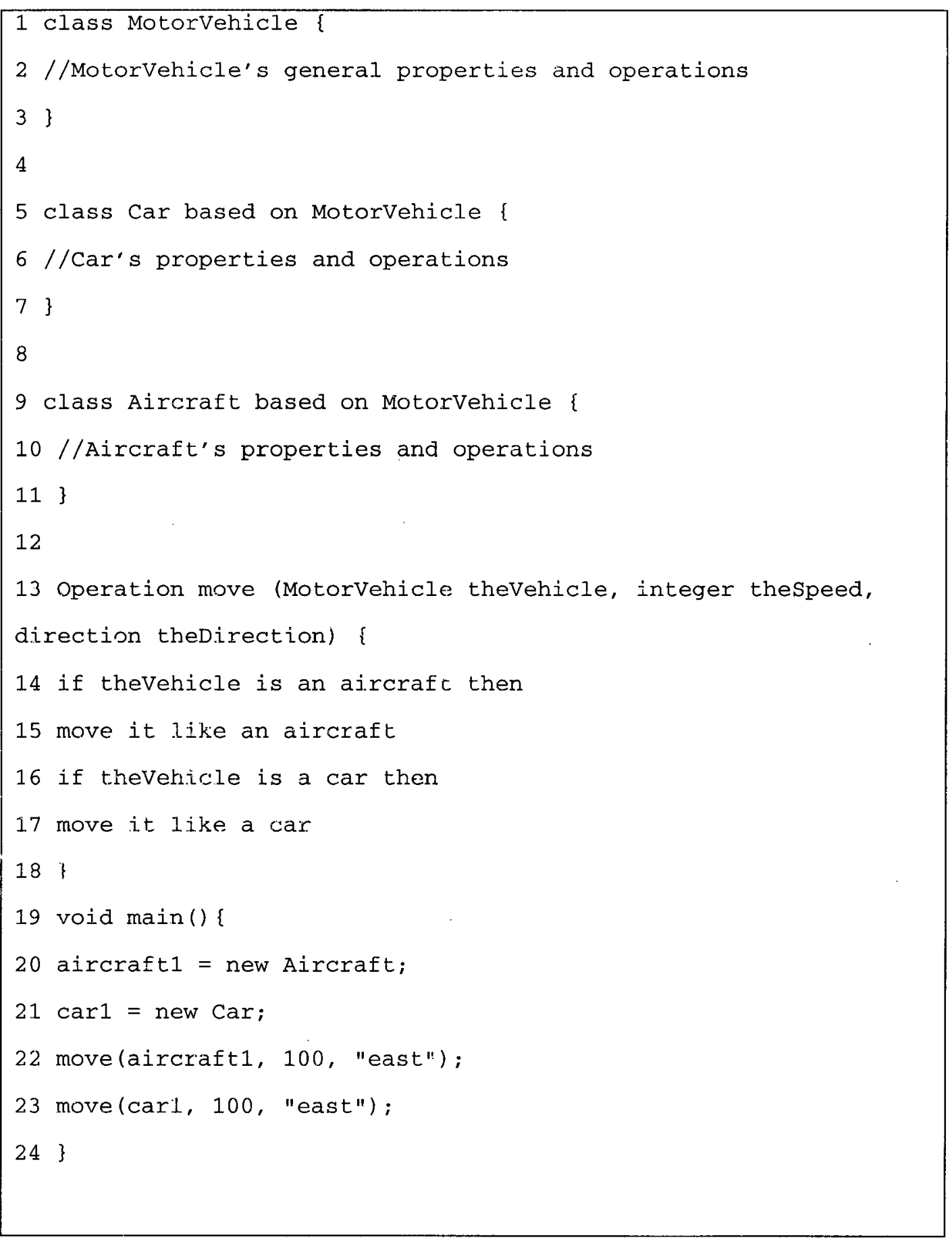

This code defines a MotorVehicle class. It then creates two subclasses of MotorVehicle: Car and Aircraft. These two subclasses inherit the properties and operations of the MotorVehicle parent class. A move operation is also declared. This operation must be passed three parameters when it is called: a mobile vehicle object, an 
integer specifying the speed, and the direction. The move operation performs different routines to set the speed and direction of the different kinds of MotorVehicles. Finally, the code example creates two objects: aircraft1 (an Aircraft) and carl (a Car). These are two different classes of objects, though both share an ancestor class of MotorVehicle. The code then uses the same operation to move both types of objects, even though the operation treats the two objects differently. To a user of the move operation, the implementation details are hidden. Moving an aircraft is the same as moving a car. This is called polymorphism.

\subsection{UML Models}

Unified Modeling Language (UML) [Rat99. BRJ99] is a popular design notation defined by Object Management Group (OMC) for object-oriented-design. The OMG specification states:

"The Unified Modelirg Language (UML) is a graphical languäge for visualizing, specifying, constructing, and documenting the artifacts of a software-intensive system. The UML offers a standard way to write a system's blueprints, including conceptuai things such as business processes and system functions as well as concrete things such as programming language statements, database schemas, and reusable software components."

The UML is important part of developing object-oriented-software and the software development process. The UML uses mostly graphical notations to express the design of software projects. Using the UML helps project teams communicate, explore potential designs, and validate the architectural design of the software. 
Each UML diagram is designed to allow developers and customers to view a software system from different perspectives and in varying degrees of abstraction. As described in [Uml03] the following UML diagrams commonly created in visual modeling tools include:

- Use Case Diagram displays the relationship among actors and use cases.

- Class Diagram models class structure and contents using design elements such as classes, packages and objects. It also displays relationships such as containment, inheritance, associations and others.

\section{- Interaction Diagrams}

- Sequence Diagram displays the time sequence of the objects participating in the interaction. This consists of the vertical dimension (time) and horizontal dimension (different objects)

- Collaboration Diagram displays an interaction organized around the objects and their links to one another. Numbers are used to show the sequences of messages.

- State Diagram displays the sequences of states that an object of an interaction goes through during its life in response to received stimuli, together with its responses and actions.

- Activity Diagram displays a special state diagram where most of the states are action states and most of the transitions are triggered by completion of the actions in the source states. This diagram focuses on flows driven by internal processing.

- Physical Diagrams

- Component Diagram displays the high level packaged structure of the code itself. Dependencies among components are shown, including source code 
components, binary code components, and executable components. Some components exist at compile time, at link time, at run times, as well as at more than one time.

- Deployment Diagram displays the configuration of run-time processing elements and the software components, processes, and objects that live on them. Software component instances represent run-time manifestations of code units.

All static and dynamic Interaction diagrams provided in this thesis are conformed to UML. For detailed description of these notations, please refer to [Boo99, Rat99]. 


\section{Chapter 3 Related Works and Thesis Problem Statement}

This research is motivated by the need for a higher-level abstraction for better understanding of software systems than design level. Design patterns represent an ideal choice because they define the micro-architecture of subsystems and components and can give a clearer overview of software systems. Hence, a pattern-level view of software systems is needed in reverse engineering.

In this chapter, the related works in this area are reviewed, followed by the problem definition, and a discussion on this problem is given in the last section of this chapter.

\subsection{The Related Works}

To date, there have been many attempts [Nie02, Wuy(01, Cag00, Cin00, Kel99, Ton99, Ant98, See98, Ban98, Shu96, Kra96, Bro96, Lan95] made to get high-level abstraction of software systems and detect design patterns.

From the view of applications, they can be divided into the following two approaches:

1. Manual pattern detection

The pattern detection is performed manually, step by step. It can only be applied in a limited range because it is very difficult and even impossible to perform this task manually for large-sized software systems.

2. Automatic pattern detection

The automatic pattern detection includes full automation and semiautomation. The source code information and the pattern definitions are 
always represented in a certain format (form), and certain query mechanisms are implemented to search for design patterns.

- Data format-based representation for pattern detection

The source code information and pattern definitions are stored in data format-based representation so that the design patterns can be detected by querying this data format base in a certain way.

- Graph-based representation for pattern detection

The source code information and pattern definitions are represented in the form of graph so as to detect design patterns by using graph query mechanisms.

- Metrics-based representation for pattern detection

The software metrics and other constraints like the structural characteristics of patterns are used to represent the sonce code and pattern definitions. Design patturns can be detected based on these.

- Logic-based representation for pattern detection The source code information is represented as logic facts and the pattern definitions as logic rules. Design patterns can be detected by querying this logic base.

These approaches are reviewed as follow's:

\subsubsection{Manual Pattern Detection}

Shull et al. [Shu96] have developed an inductive method to help discover custom domain-specific design patterns in existing OO software systems, which is called BACKDOOR (Backwards Architecting Concerned with Knowledge Discovery of OO 
Relationships). Creating a pattern knowledge base for a development organization as shown in Figure 3-1 motivates their research.

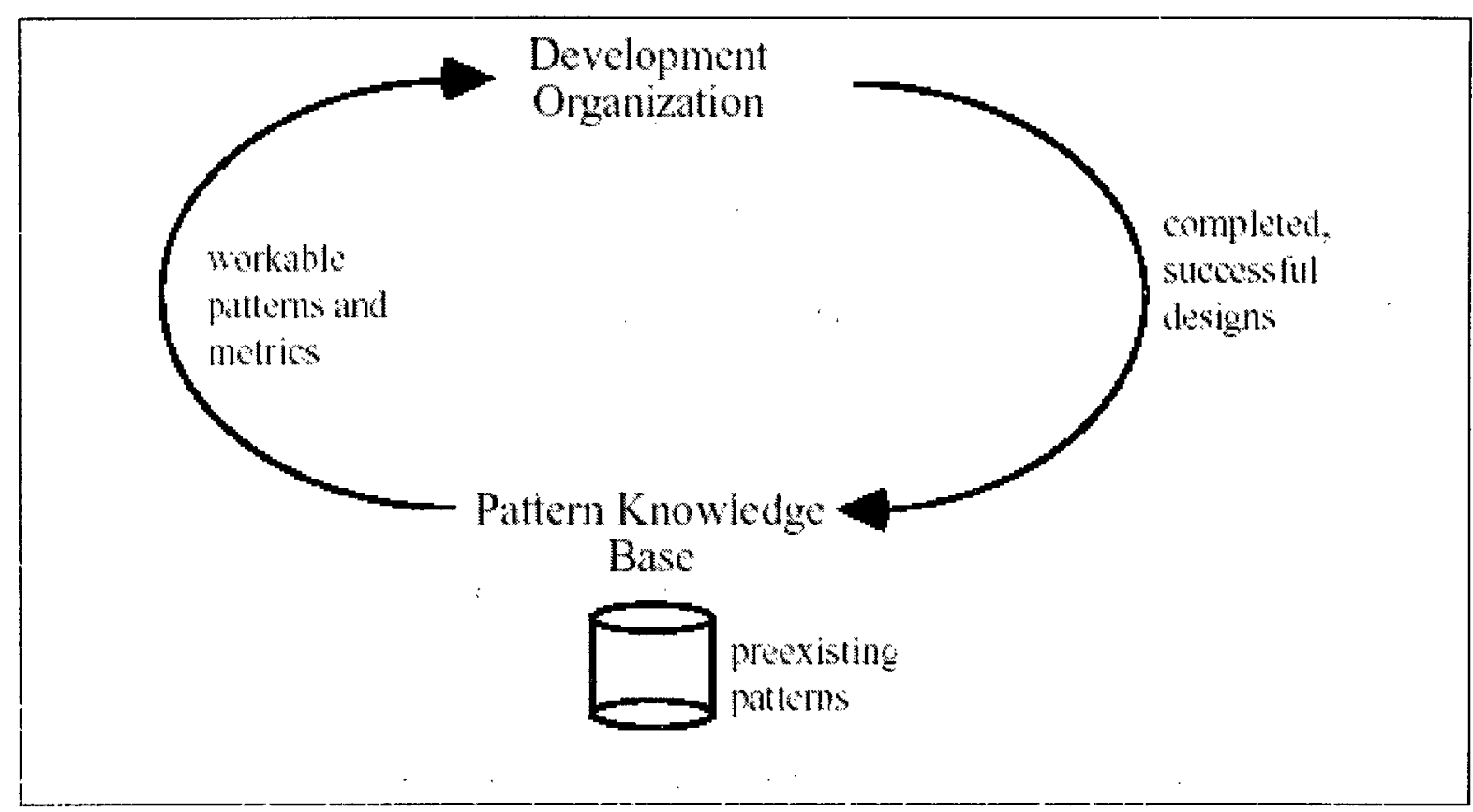

Figure $3-1$ The cycle between $\mathrm{DO}$ and $\mathrm{PKB}$

This goal can be achieved by multiple iterations through a feedback cycle between the Domain Organization (DO) and Pattern Knowledge Base (PKB).

Their research aim is to discover design patterns, which is called reverse architecting design patterns, not to recover existing design patterns as we do in this thesis.

BACKDOOR consists of six steps, which are really iterated within and across steps. It starts by reviewing the problem specification and design documents, then develop and refine the model of the system, finally, identify potential candidates for patterns based on inheritance and communication links between classes.

Shull et al. work gives some hints about how to extract design patterns from the source code, but the whole process is performed manually. It will become a very tough task when a big-sized software system is analyzed. 


\subsubsection{Automatic Pattern Detection}

The automatic pattern detection discussed in this section encompasses full automation and semi-automation.

\section{1. 2. 1 Data format-based presentation for pattern detection}

[Ke199, Sch99] Keller and Schauer et al. present an environment called SPOOL for the reverse engineering of design components based on the structural descriptions of design patterns. This process of pattern detection encompasses the following parts: source code capturing, design repository, pattern-based design recovery and design representation. Source code is parsed by a $\mathrm{C}++$ parser then the source code elements are represented by an ASCII-based representation UML/CDIF as an intermediate representation. The source code model and the abstract design components that are recovered are stored in a design repository, a data format base. The abstract design components are actually the structural descriptions of design patterns. A query mechanism that can recognize the structural descriptions in the source code models are implemented to extraci design patterns from this database. The detected patterns are visualized through design representation to provide an intuitive impression for human analysis and better understanding the software system.

In their approach the representations of design patterns are only based on the structural characteristics of design patterns. Both the structural and behavioral characteristics are considered in our approach. Their approach doesn't propose a generic pattern detection algorithm like ours does. They do not report on how to deal with relationships among classes, which has a great impact on identifying design patterns. 


\section{1.2.2 Graph-based presentation for pattern detection}

Niere et al. [Nie02] propose an approach to solving the implementation variants problem by defining sub-patterns using a formal description ASG (Abstract Syntax Graph) to describe design patterns.

The parsed program information is obtained from JavaCC parser and converted into ASG objects as an intermediate representation. The design patterns and sub-patterns are defined as rules. These rules are used to search for pattern instances from ASG objects that are obtained from source code by switching between bottom-up and topdown search strategy.

In their approach to deal with implementation variants they defined many subpatterns to reflect the relationships among classes such as association, aggregation and so on. In order to overcome the problem of many definitions of slightly different variants of subpatterns a general rule has been used to replace several slightly different subpattern rules. This consists of the common parts of those replaced rules, but it leads to more false positives. Therefore, a balance between the number of general rules and the number of detected false positives has to be determined by the reverse engineer. In our approach, the parser has been developed to directly deal with implementation variants of sub-patterns (i.e., relationships among classes).

\section{1. 2. 3 Metrics-based representation for pattern detection}

Antoniol et al [Ant98] describe a multi-stage filtering process to detect design patterns, in which multiple metrics of patterns and design patterns are described through their structural characteristics, e.g. the number of classes, the number of inheritances, and the number of references, etc. At each stage different metrics and other constraints like 
delegation (a kind of class relationship) are used to reduce the search space. The reason for using metrics in pattern definitions is to reflect the quality of source code in software metrics. For the recovery of design patterns, this may lead to many false positives due to the quantitative definition of design patterns. In the Chapter 6 case study, in this thesis, a comparison table of extraction results between their approach and ours is provided to show that our approach does reduce the false positives for the same pieces of sample code.

\section{1.2. 4 Logic-based pattern detection}

Kramer et al. [Kra96] propose an approach based on Prolog queries. The Prolog descriptions of several patterns are provided, and the parser Paradigm Plus is used to parse $\mathrm{C}++$ header files. However, the front-end paiser could not parse $\mathrm{C}++$ definition file and has not defined a complete Prolog representation to describe design patterns. Therefore some key characteristics of design patterns get lost, and it leads to a lot of false positives, e.g. delegation, a kind of class relationship and a special furm of association. In [Kra96] delegation relationships are replaced by association relationships, so more false positives have been raised.

K. Mens and R. Wuyts et al. [Me01, Men01, Wuy98, Wuy01] adopt a declarative meta-programmıng approach to explicitly codify programming patterns in software development process. They reify the structure of object-oriented programs in terms of logic clauses, and declare programming patterns as logic rules on top of these clauses. By querying the logic system, these rules allow for checking, enforcing and searching for occurrences of patterns in the software. The approach uses Prolog query to identify 
design patterns in terms of a description of design patterns based on the structure of an OO program.

In their approach, the basic program information is directly represented as logic facts. This may make the logical programs become too big for large-sized software systems. Consequently, the rules become quickly impossible to manage. In our case, we separate relationship detection algorithms from pattern detection. Doing so can reduce the search space from both the fact base and the rule base. In addition, dynamic characteristics of design patterns are not discussed in their papers though it is important in identifying some behavioral design patterns.

In [Ric99] Richner et al. propose a logic $\cdots$ based approach to get high-level view of software systems, by creating high-level abstractions to produce views based on the combination of user-defined queries. Static and dynarnic characteristics of software systems are represented as logic facts. By making different queries on the facts, the user can decide what kind of views will be produced. In [Ric99], the approach is similar to ours since both static and dynamic information of Object-Oriented software systems have been taken into account and both are based on the logic programming language Prolog. Their goal is to achieve a high-level view of software systems at the design level, but we focus on getting higher-level abstraction at the pattern level. They did not consider getting pattern level abstraction like ours and did not construct a model for the formal descriptions of design patterns.

Eden, Gil and Yehudai et al. [Ede97, Ede98, Ede99] have developed and used a declarative pattern specification language of design patterns-LePUS to formalize the design patterns. This formal specification of design patterns can be used as a tool in 
support of the implementation and recognition of design patterns. It is helpful extracting design information using design patterns, but some rules in the formal specification are not suitable for identifying design patterns from source code. For instance, the Observer patterns mentioned in [Ede99] is formalized using class hierarchy and invocation of methods. However, the behavior of the sets of classes in Observer is hard to be captured merely depending on the static information directly obtained from source code. From our point of view, some behavioral design pattems like Observer can be effectively identified based on dynamic information of software systems.

\subsection{Problem Definition in This Thesis}

In order to get a pattern-level view of software systems detecting design patterns in software systems is a must. This is exactly the problem we focus on in this thesis.

The related works mentioned above are also concerned with it to a certain extent. However, there are some points that needed to be improved upon in their different approaches.

- Shuell et al [Shu96] propose to discover design patterns in software systems manually. But manual pattern detection has turned out to be impossible for large-sized software systems.

- Keller and Schauer et al.[Ke199,Sch99] extract design components based on the structural descriptions of design patterns. However, some design patterns can be effectively identified by looking at the behavioral characteristics of design patterns. 
- In the approach proposed by Antoniol et al [Ant98], the metrics used can lead to many false positives due to the quantitative definition of design patterns.

- The front-end parser used by Kramer et al. [Kra96] could not parse C++ definition file, and has not defined a complete Prolog representation to describe design patterns.

- In the approach proposed by $\mathrm{K}$. Mens and R. Wuyts et al. [Me01, Men01, Wuy98, Wuy01], the program information is directly represented as logic facts. This may make the logical programs too big to manage for large-sized software systems.

- Richner et al. [Ric99] focus on getting high-level view of software systems at design level, not at partern level.

- In the formal specification of design patterns proposed by Eden, Gil and Yehudai et al. [Ede97, Ede98, Ede99], some rules are not suitable for identifying behaviorai design patterns, like Observer from source code.

To avoid these drawbacks a rule-based approach is proposed to detect design patterns in this thesis. The model of formal descriptions of design patterns is presented and is based on both static and dynamic characteristics of design patterns. In addition, the parser developed in this thesis can analyze the entire $\mathrm{C}++$ code and not the header file alone. This approach is described in details in Chapter 4 and Chapter 5 presents the prototype. Two major case studies are reported in Chapter 6. 


\subsection{Summary}

Design patterns can be taken as micro-architectures for solving particular design problems because design patterns reflect the structure and behavior of a set of classes in a software system. Especially when the design documentation is poorly recorded, reverse engineering software systems can detect design patterns to achieve a high-level view of software systems because design patterns reflect interrelationships among a set of classes. Of course, not all software systems contain design patterns in their source code, e.g. some legacy code developed before emergence of the concept of design patterns. However, because design patterns record experts' experience and enable novices to create supreme design, they have been widely used in software development process for reuse. The detection of design patterns in software systems would be very helpful for software maintenance. 


\section{Chapter 4 An Approach to Design Pattern Detection}

\subsection{Introduction}

As mentioned in Chapter 3, the problem to be solved in this thesis is how to detect design patterns in software systems. There are three factors that have a huge impact on this issue:

- The front-end parser for extracting program information

- The detection algorithms for recovering design information

- The pattern detection algorithms for identifying design patterns.

The precision of program information provided by the front-end parser directly influences the result of extraction. A parser that can scan both header files and definition files in $\mathrm{C}^{++}$code is what is needed since it can previde complete program information about source code.

Design information, such as classes and their interrelationships, is the foundation of identifying design patterns. Because UML relationships are defined from the perspective of design, some relationships may map too many implementation forms in the source code. Sometimes this may cause many faise pattern instances, and even ruin the process of pattern detection. So a refinement of these relationships is needed and is actually equivalent to finding effective detection algorithms for recovering design information.

Pattern detection plays an essential role in identifying design patterns. Pattern detection algorithm should be effective for all kinds of GOF design patterns.

According to the factors above the following work has been conducted, 
- A C++ parser has been developed. It is able to examine the entire source code, i.e., header and definition files.

- A set of detection algorithms based on the relationships among the different components of the software system has been developed.

- A model for formal description of design patterns has been proposed. Design patterns based on static and dynamic information are modeled in a logic language.

- A rule-based approach for pattern detection is adopted.

The logic language has the following advantages:

- The absence of control structures in logic language results in a simple, easy to learn and easy to use language.

- Using a logic programming language to express queries has advantages in that, although the queries are very powerful the language is easy to understand and use.

- The logic language is open in such a way that everyone can easily add information to the unification process, which makes the DPET tool more flexible (The DPET tool is described in Chapter 5). This is exactly suitable for development of design patterns because more patterns would be discovered continuously. It would be easy to add new rules to the rule base for identifying design patterns.

The major disadvantage of logic programming languages is the slow execution time. This depends on the complexity of the query that needs to be solved. When a large software system that often includes several hundred classes is analyzed, if the relationships among them are complex the execution time will be long. In order to avoid this, we separate design information detection from design pattern recovery in the 
implementation. The algorithms for detecting the relationships among classes are implemented in $\mathrm{C}++$, rather than logic language Prolog.

In this approach design patterns are modeled based on static and dynamic information in logic language.

Static information about design patterns can be obtained more easily than dynamic information. Sometimes, however, dynamic information about design patterns is the key to identifying behavioral design patterns. For instance, the Observer design pattern is hard to identify merely in terms of a structural-based model (i.e. static information), which leads to so many false positives that it may ruin the detection. What we can know about dynamic information from the structure-based model is which object invokes another object between two operations. It is hard and even impossible to obtain the information about the sequence of messages exchanged among multiple objects merely by analyzing the source code statically, because of some of the features of $O O$ programming like polymorphism.

\subsection{Formal Description of Components and Relationships}

In our model the design information extracted from a software system consists of components and the relationships among them.

Components are of two kinds, static components and dynamic components. Static components consist of classes and operations while dynamic components are composed of objects, that is, the instances of classes during run time.

Relationships consist of static relationships and dynamic relationships. Static relationships include Inheritance, Aggregation, Using, Association, and Delegation. 
Dynamic relationships include CreationLink, SendMessage, and SelfMessage.

DIS: Design Information in Software;

C: Components; SC: Static Components; DC: Dynamic Components;

R: Relationships; SR: Static Relationships; DR: Dynamic Relationships;

DIS $\supset\{C, R\} ;$

$\mathrm{C} \supset\{\mathrm{SC}, \mathrm{DC}\}$

$\mathrm{SC} \supset\{$ Classes (abstract class, concrete class). Operations (abstract and concrete operations)\},

DC $\supset\{$ Objects (instances of classes) $\}$;

$\mathrm{R} \supset\{\mathrm{SR}, \mathrm{DR})$;

$\mathrm{SR} \supset\{$ Inheritance, Aggregation, Using, Association, Delegation $\}$;

DR $\supset\{$ CreationLink, SelfMessage, SendMessage \};

Table 4.1 The Composition of the nodel for formal description of design patterns

As for a design pattern its static characteristics focus on a set of static comporients

including classes, operations, and static relationships; while its dynamic characteristics

(behavior) focus on a set of dynamic components including objects and dynamic

relationships, i.e., messages exchange betweer them.

The definition of relationships and their detection algorithms are as follows.

\subsubsection{Relationships}

\subsubsection{Static Relationships}

The definitions of static relationships in this section are based on the definitions in [Boo94].

\section{- Inheritance:}

Inheritance is the mechanism that allows a class to inherit properties of another class. If class A inherits from class B, then B is called superclass of A. A is called 
subclass of B. Objects of a subclass can be used where objects of the corresponding superclass are expected. This is due to the fact that objects of the subclass share the same behavior as objects of the superclass. Objects of the inheriting class have access to attributes and operations of the inherited class without the need to redefine them.

Multiple inheritances mean that one subclass can have more than one superclass. This enables the subclass to inherit properties of more than one superclass and to "merge" their properties.

- Implementation: Finding inheritance by examining the declarations or definitions of classes according to the detection algorithm in Table 4.2

Table 4.2 Detection algorithm of Inheritance in C++ pseudo code.
These relationships are identified according to declaration of the following forms, these
declarations are asually found in the header files:
class Class_A: Class_B
class Class_A: public Class_B
class Class_A: protected Class_B
class Class_A: private Class_B
class Class_A: virtual Class_B
If (Class_B: public/protect/private Class_A $\{\ldots\})$
Class_B inherits from Class_A.
Multiple inheritance:
If ( Class_C:public/protect/private Class_A,CClass_B $\{. .\}.) / *$ Multiple inheritance*/
Class_C inherits from Class_A and Class_B.
Note that multiple inheritance and virtual inheritance are also taken into account in the
tool DPET.




\section{- Aggregation:}

Aggregation asserts a direction to the whole/part relationship. It can be divided into two kinds of containment [Boo94]. One is containment by value, a kind of physical containment meaning where the object does not exist independently of its enclosing instance. Another less direct kind of aggregation is also possible, called containment by reference. For example, Class_A has a data member $b$ that is a pointer to Class_B with the following declaration:

class Class_A

Class_B * b;

$\| \ldots$

\}

In this case the enclosing class still denotes the whole and an instance of the enclosed class is still one of its parts, although that part must now be accessed indirectly. Hence, the lifetime of each of these two objects are not so tightly coupled as before: we may create and destroy instances of each class independently.

- Implementation: Typically the aggregation relationship is detected by looking for data-member declarations in classes that are of other user-defined class types as shown in Table 4.3.

Table 4.3 Detection algorithm of Aggregation in C++ pseudo code.

- Class_A has Class_B by value.

class Class_Á

Class_B b;// an instance of Class_B is part of Class_A

Class_C c cm]; //an array of instances of Class_C is part of Class_A

$11 .$.

\} 
- Class_A has Class_B by reference

class Class_A\{

Class_B b*; // a dynamic instance of Class_B is part of Class_A

Static Class_C $* \mathrm{c}[\mathrm{m}]$; //a dynamic array of instances of Class_C is part of Class_A $\|$...

Class_A (); //constructor for Class_A

Class_A::Class_A \{

Class_B $b=$ new Class_B; // dynamically creating $b$;

Class_C c []$=$ new Class_C; //dynamically creating $\mathrm{c}[]$;

- Template A has Class_B

template $<$ Class_B $>$ A; / template instantiation

If ( A has B by value $\|$ A has B by reference $\| \mathrm{A}$ has B in template )

A aggregates $B$.

- Composition:

We did not take composition relationships, separately, into account when we carried out the recovery of design pattern. Composition is a specific kind of aggregation and the difference between aggregation and composition is only focused on meaning of code. It is hard and even impossible to discern their difference from the syntax of the code without human involvement when using an automated tool.

\section{- Using Relationships}

"Using" relationships among classes parallel the peer-to-peer links among the corresponding instances of these classes. Whereas an association denotes a bi-directional semantic connection, a "using" relationship is a possible refinement of an association, whereby we assert which abstraction is the client and which is the supplier of certain services [Boo94]. 
In general, a using relationship is a unidirectional association with the "using" refining the association. Strict "using" relationships are occasionally too confining because they only allow the client the public interface of the supplier. Sometimes, for tactical reasons, we must break the encapsulation of these abstractions, which is the very purpose of the friend concept in $\mathrm{C}++$.

- Implementation: This relationship is generally implemented through method parameters and therefore detected by examining method parameter types as shown in Table 4.4.

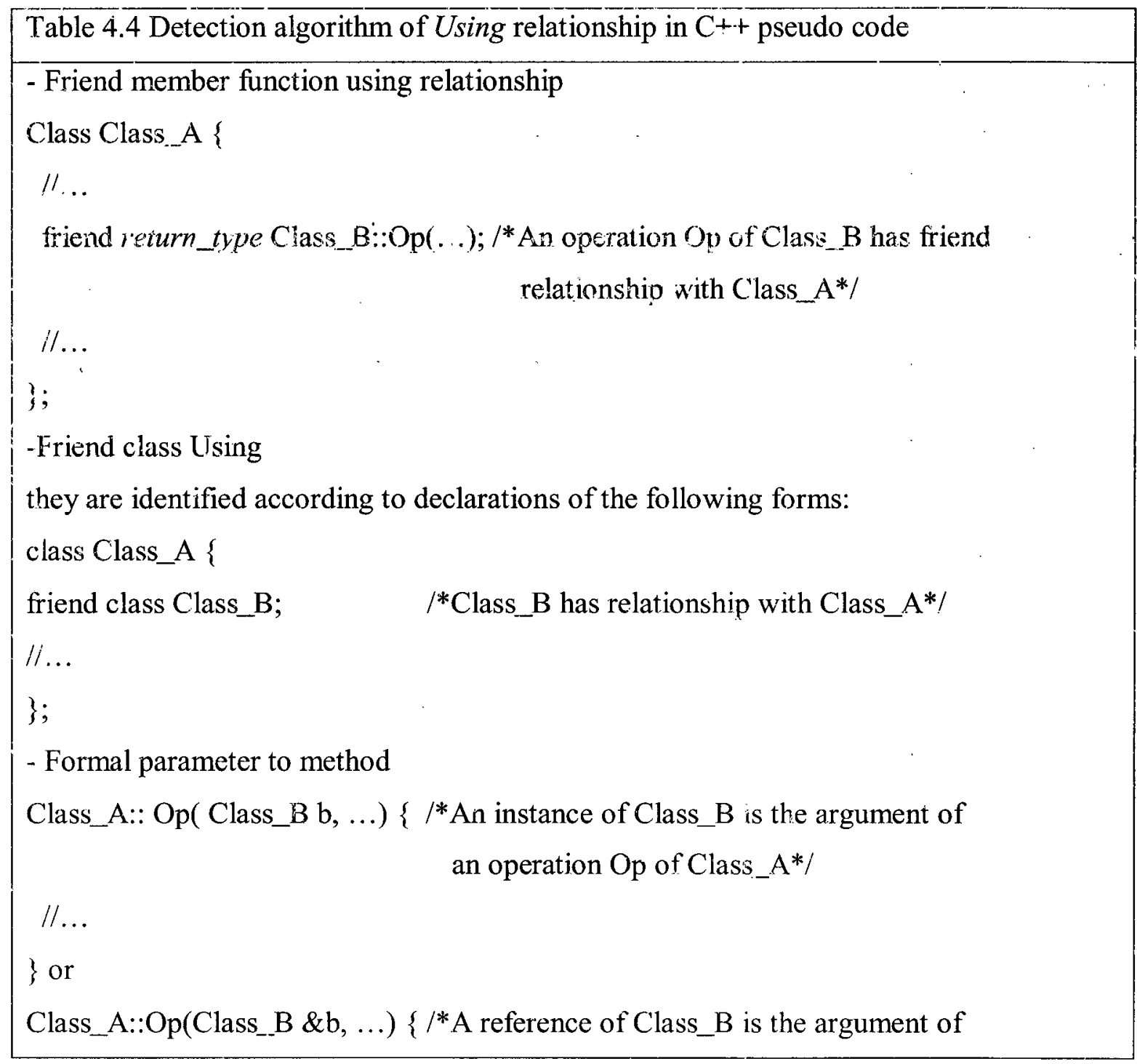




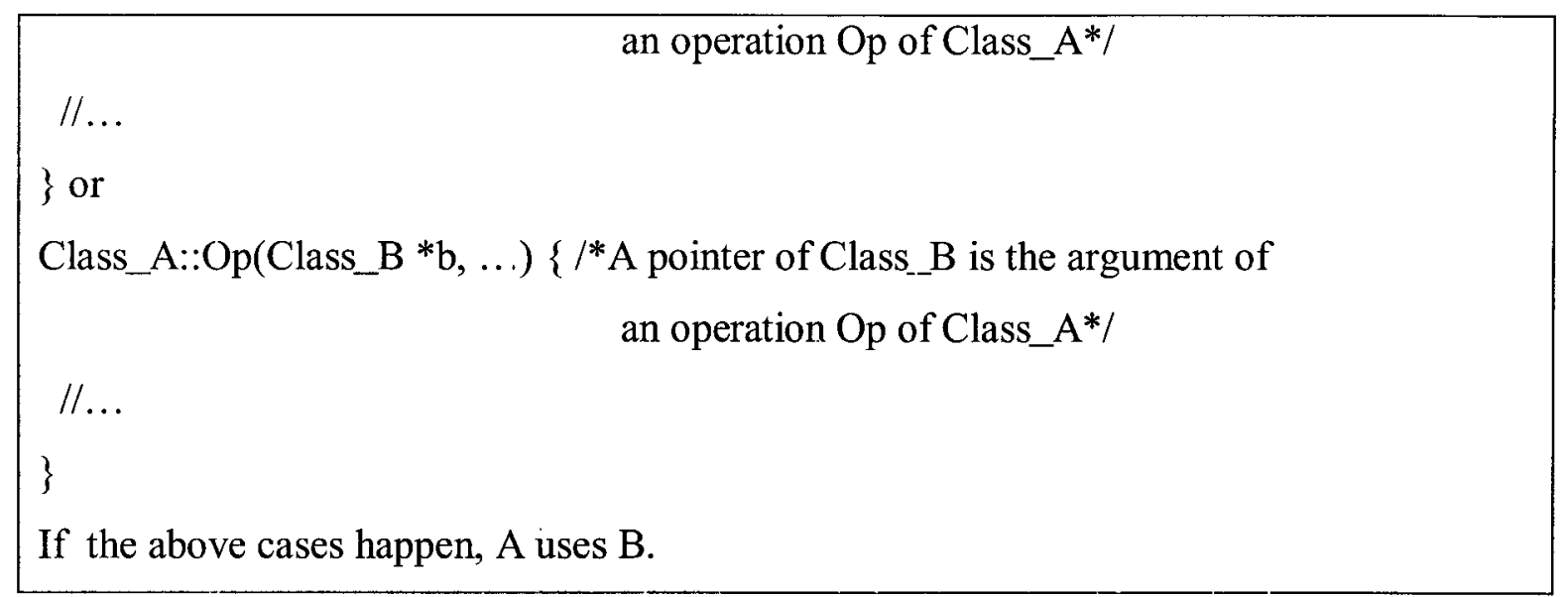

\section{- Association:}

An association is often replaced by cyclic aggregation or cyclic "using" relationships [Boo94]. More often than not, however, an association (which by definition implies bidirectional navigation) is refined during design to be a single aggregation or "using" relationship, thus denoting a constraint upon the direction of the association. Multiplicity: One-to-one; One-to-many, Many-to-many;

- Implementation: Cyclic aggregation or cyclic using relationships as shown in Table 4.5.

Table 4.5 Detection algorithm of Association in pseudo code. If ( $\mathrm{A}$ uses $\mathrm{B} \& \& \mathrm{~B}$ uses $\mathrm{A}) \|(\mathrm{A}$ aggregates $\mathrm{B} \& \& \mathrm{~B}$ aggregates $\mathrm{A}$ ) A associates with B.

\section{- Delegation:}

A specific application of an object composition is a delegation, an object that is receiving messages, a delegator, delegates these requests (received messages) to a 
delegatee object. Often combined with inheritance, delegation is heavily used in design patterns.

Delegation is a way of making composition as powerful for reuse as inheritance. In delegation two objects are involved in handling a request; a receiving object delegates operations to its delegatee. This is analogous to subclasses deferring requests to parent classes.

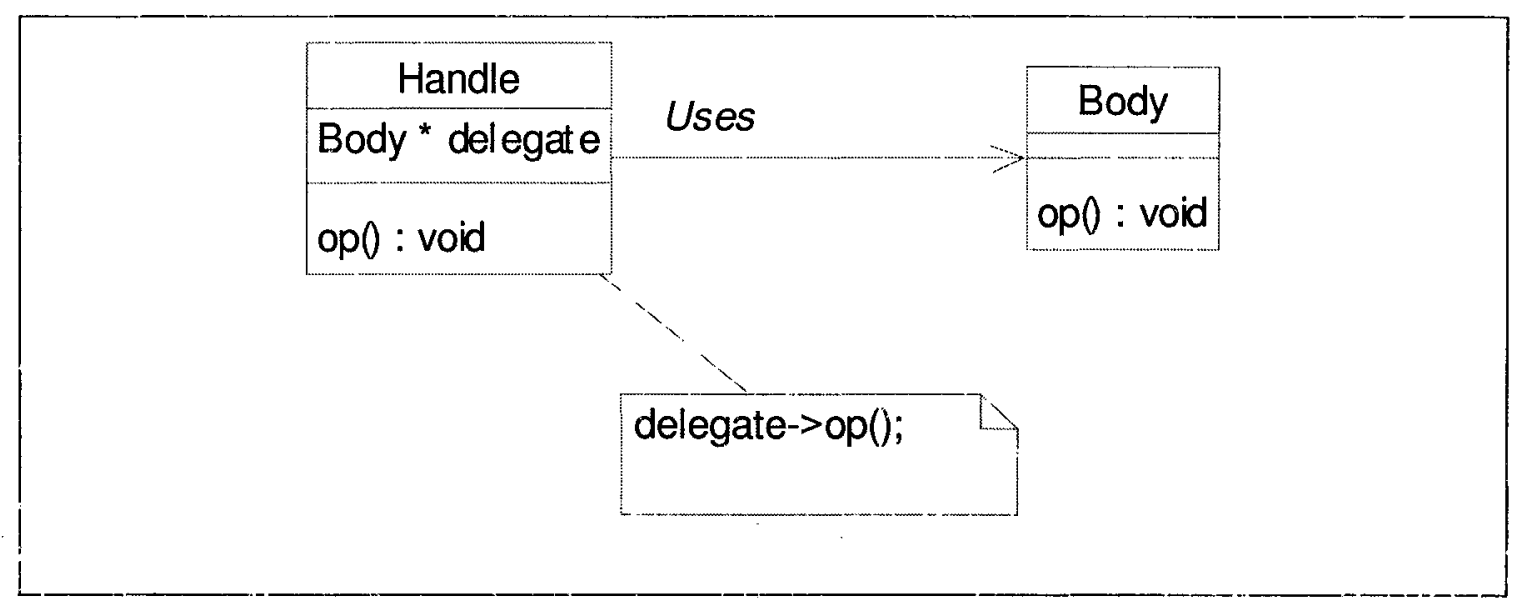

Figure 4-1 UML class diagram of Delegation relationship

- Implementation: From the above figure 4-1, we can see that there are two classes. One class "Handle" contains a pointer of class "Body" as its attribute. The member function op() of class "Handle" is implemented by the member function op() in class "Body". This is a kind of object composition by a pointer and actually a Handle/Body idiom in $\mathrm{C}++$, which has been widely used in $\mathrm{C}++$ coding. The handle represents an abstraction and it is visible to the client, while the invisible body provides the implementation. The handle-body technique results in another design pattern, called the "Bridge design pattern". Many design patterns have this kind of interclass relationship such as "Adapter", "Proxy", "Composite" and so on.

Table 4.6 Detecting Delegation algorithm in C++ pseudo code. 


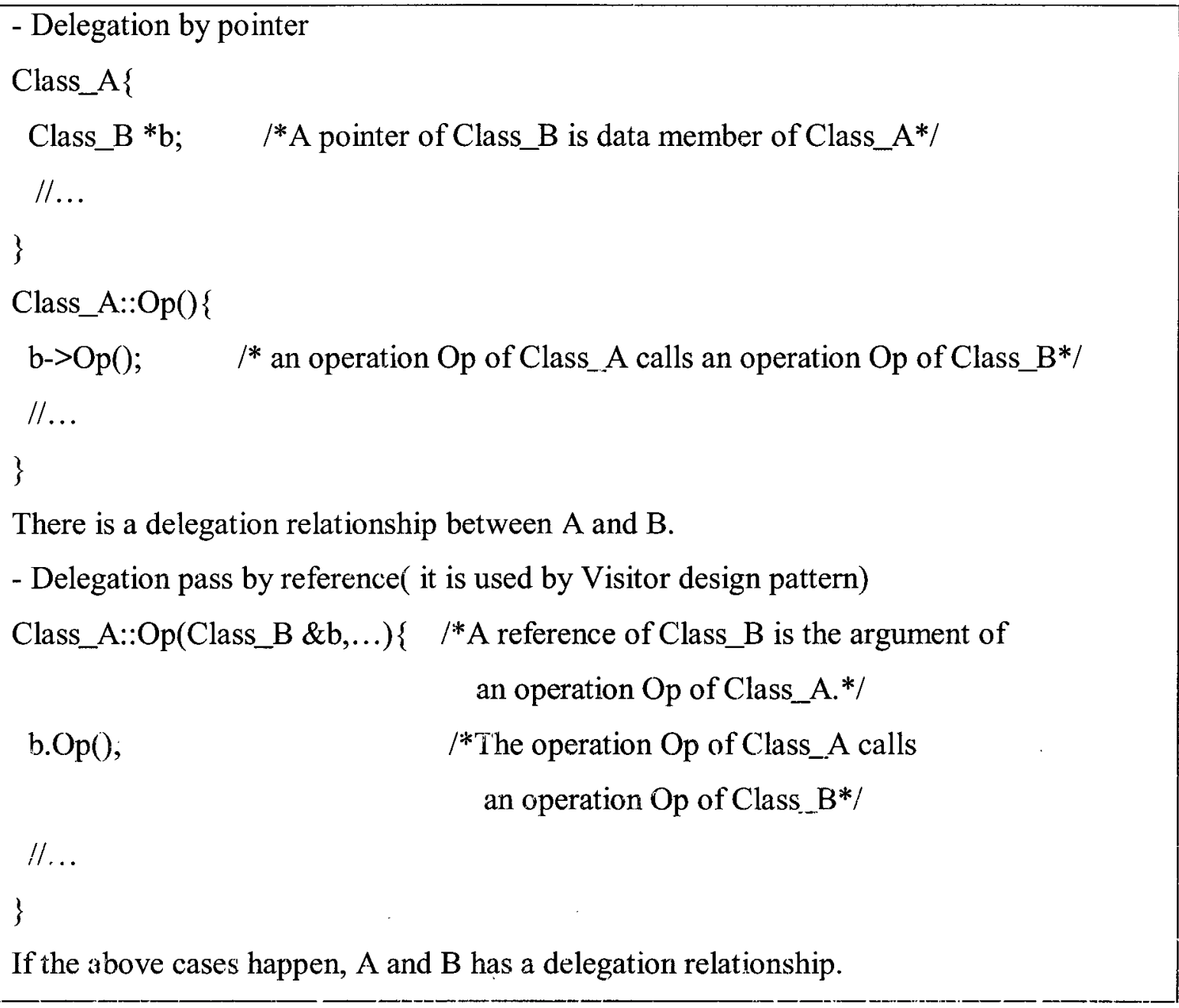

\section{2. 1.2 Dynamic relationships:}

\section{- CreationLink:}

During run time, one object may create other objects, e.g., the instance of Class Factory in Abstract Factory design pattern is responsible for creating all the instances of other classes.

- Implementation: The instance of Class A creates an instance(s) of class B.

Though we cannot know when the instance of a class is created from source code, we can get CreationLink relationships merely from source code as shown in Table 4.7. 
Table 4.7 Detecting CreationLink algorithm in C++ pseudo code

If $(\mathrm{A} \leftarrow \mathrm{B}:: \mathrm{Op}() \& \&$

$\mathrm{B}:: O p()\{$

$\mathrm{A}$ (derived) Ptr = new A(derived); return Ptr;

or return new A(derived);..... $\}$ )

CreationLink(B,A(derived));

$\mathrm{A} \leftarrow \mathrm{B}:: \mathrm{Op}()$ : the return type of $\mathrm{B}: \mathrm{Op}()$ is $\mathrm{A}$.

\section{- SendMessage:}

This relationship can be only acquired by dynamic analysis because the sequence number of sending messages can be known only when executing the program. Through static analysis the function calls between two functions (methods) may be obtained, but no object information is available for use. In comparison with sequence diagrams, a call graph is easier to construct by profiling the source code. Call graphs, however, only provides us with the information on function calls between two objects, but we cannot be aware of the sequence of messages exchanged among objects. Therefore, an appropriate dynamic analysis tool may be required to offer a variety of sequence diagrams.

\section{Table 4.8 Detecting SendMessage algorithm in pseudo code}

If $($ ObjectA sends a message to ObjectB and

the sequence number of the message is known )

ObjectA sends a Message to ObjectB in Sequence Number. 
- SelfMessage

This is a case where one object sends a message to itself instead of other objects.

Table 4.9 Detecting SelfMessage algorithm in C++ pseudo code

If $($ Class_A::Op() \{ Class_A::anotherOp(); $\}$ )

The Object sends a message to itself, that is, one operation in Class_A invoke another operation in the same class.

\subsection{Prolog Representation of the Formal Description}

In this section, we present a Prolog representation of the formal description given in section 4.2 .

\subsubsection{Components}

A program is defined as a collection of components and the relaticnships among them. Components consist of static components, dynamic components and relationships that consist of static relationships and dynamic relationships.

\section{3.1. 1 Static Components:}

- Class

class(ClassType, ClassName)

If a software component is class, and class ClassName is of type ClassType.

Note: Class Type includes concrete classes and abstract classes 
- Operation

operation(Category,Kind,Class,Name,VisibilityScope,Params,ReturnType)

There is an operation with various properties in Class.

Category: virtual, pure virtual, concrete;

Kind: selector or modifier;

Class: class name enclosing the operation;

Name: operation name;

Visibility Scope: public, protect, and private;

Params: parameters of the operation;

ReturnType: The return type of the operation.

\section{3. 1.2 Dynamic Components:}

\section{- Object}

object(Class Type, InstanceName)

A component of InstanceName is an object and it is the instance of a ClassType class.

\subsubsection{Relationships}

Relations consist of static relationships and dynamic relationships (behavior).

\subsubsection{Static relationships}

Static relationships include inheritance class hierarchy, aggregation, using, association, and delegation.

\section{- Inheritance}


inheritance(SuperClass, SubClass)

SubClass inherits from SuperClass in class hierarchy.

- Aggregation

aggregation(Aggregator, Aggregatee, Multiplicity).

Aggregator aggregates Aggregatee with Cardinality. Multiplicity: one-to-one, one-to-many, many-to-many

- Using

using(ClassNameA, ClassNameB)

ClassA uses ClassB.

- Association

association(ClassNameA, ClassNameB)

Class A associates with ClassB.

- Delegation

Delegation(ClassNameA, ClassNameB)

Class A delegates ClassB;

4. 3.2.2 Dynamic Relationships:

- CreationLink

sreationlink(ClassNameA, ClassNameB)

The instance of class $\mathrm{A}$ is created by an operation of class $\mathrm{B}$ 
- SendMessage

sendmessage (ObjectA, ObjectB, MessageA, SequenceNumber)

Message $A$ is sent from Object $A$ to ObjectB in the order of sequence number.

Note that the sequence number is the sequence of message exchange.

- SelfMessage

selfmessage (Object, Message, SequenceNumber)

Object sends a Message to itself.

Note that the sequence number is the sequence of message exchange. 


\subsection{Formal Description of Design Patterns}

This section describes the model of a formal description of design patterns in Prolog. The following typical patterns from GOF design patterns are selected as examples,

- Creational design patterns: Factory Method.

- Structural design patterns: Adapter, Composite, Decorator, Proxy, and Bridge.

- Behavioral design patterns: Strategy, Observer, Chain of Responsibility, and Visitor. All the models consist of components and relationships defined in our model in section 4.2. Different kinds of design patterns may have different compositions of the model. For example, Structural design patterns contain strong structural characteristics, so the models for identifying them are just based on static information. But it is not the case to some creational design patterns. Some creational design patterns, like the Factory Method, cannot be identified merely based on static information. Instead, dynamic information like CreationLink, along with static information can be used to detect Factory Method design patterns from $\mathrm{C}++$ code. For behavioral design patterns, some, such as Chain of Responsibility and Observer are easier to identify if their models are based on dynamic information because they are of strong behavioral characteristics. Meanwhile, in order to address the key role of dynamic information in identifying behavioral design patterns theObserver pattern's structure-base model based on static information is introduced for comparison with the behavioral-based model based on dynamic information. The impact of both cases is discussed later in this section. 


\subsubsection{The Models of Creational Design Pattems}

\section{4. 1. 1 Factory Method}

The Factory Method defines an interface for creating an object, but allows subclasses to decide which class to instantiate. Actually, Factory Method lets a class defer instantiation of subclasses. Factory Method design pattern is based on both static and dynamic information. Factory Method can be applied to defer the instantiation of classes, that is, the task of creating objects to be forwarded to the subclass whose superclass includes the operations responsible for creating objects. Sometimes the superclass provides a default operation for instantiation of classes, rather than do nothing.

Figure 4-2 is a UML class diagram of Factory Method design pattern. Its structure is composed of two class hierarchies. Using the sign "new " in $\mathrm{C}++$ code, the CreationLink relationship defined in section 4.2.2.2.1 can be found to show a link between Product class and Creator class, and another link between ConcreteProduct class and Concreator class. This way, Factory Method design patterns can be extracted from source code effectively. The detailed extraction process will be described in Chapter 5 .

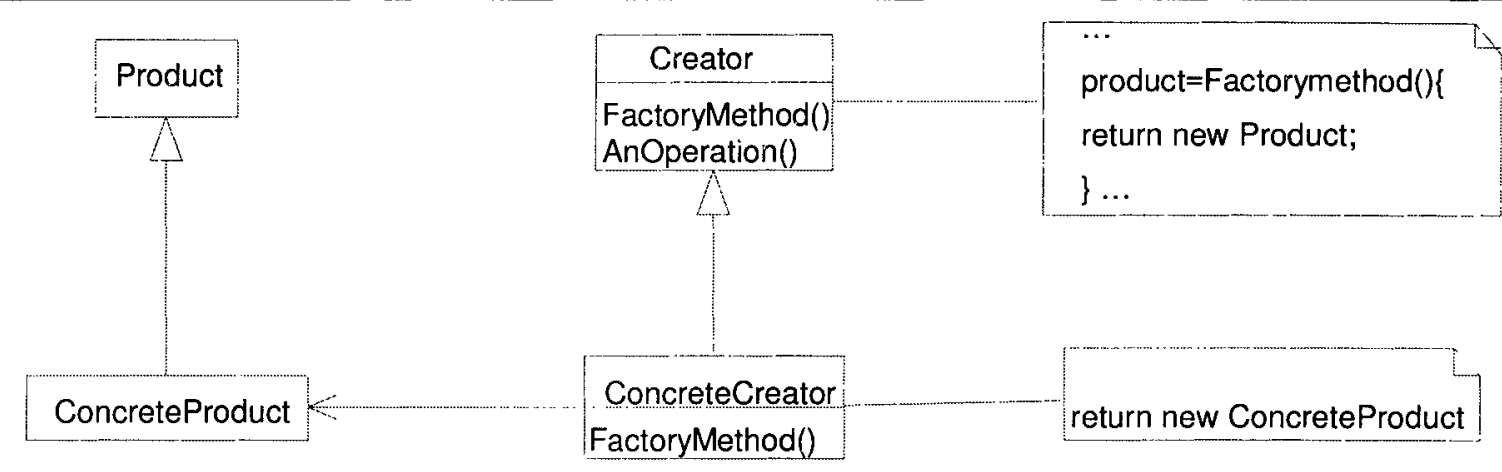

Figure 4-2 UML class diagram of Factory Method design patterns 


\subsection{The mapping between the model of Factory Method and its Prolog representation}

The mapping between the model of Factory Method and its Prolog representation is as follows,

\begin{tabular}{|l|l|}
\hline Elements in the model & Prolog representation \\
\hline Static Components & fm_classes \\
Classes & fm_ops \\
Operations & fm_stcrelations \\
\hline Static Relationships & inheritance \\
Inheritance & fm_dynrelations \\
\hline Dynamic Relationships & creationlink \\
\hline CreationLink & \\
\hline
\end{tabular}

Using Figure 4-2 and the table above, the model of Factory Method in Prolog is as follows:

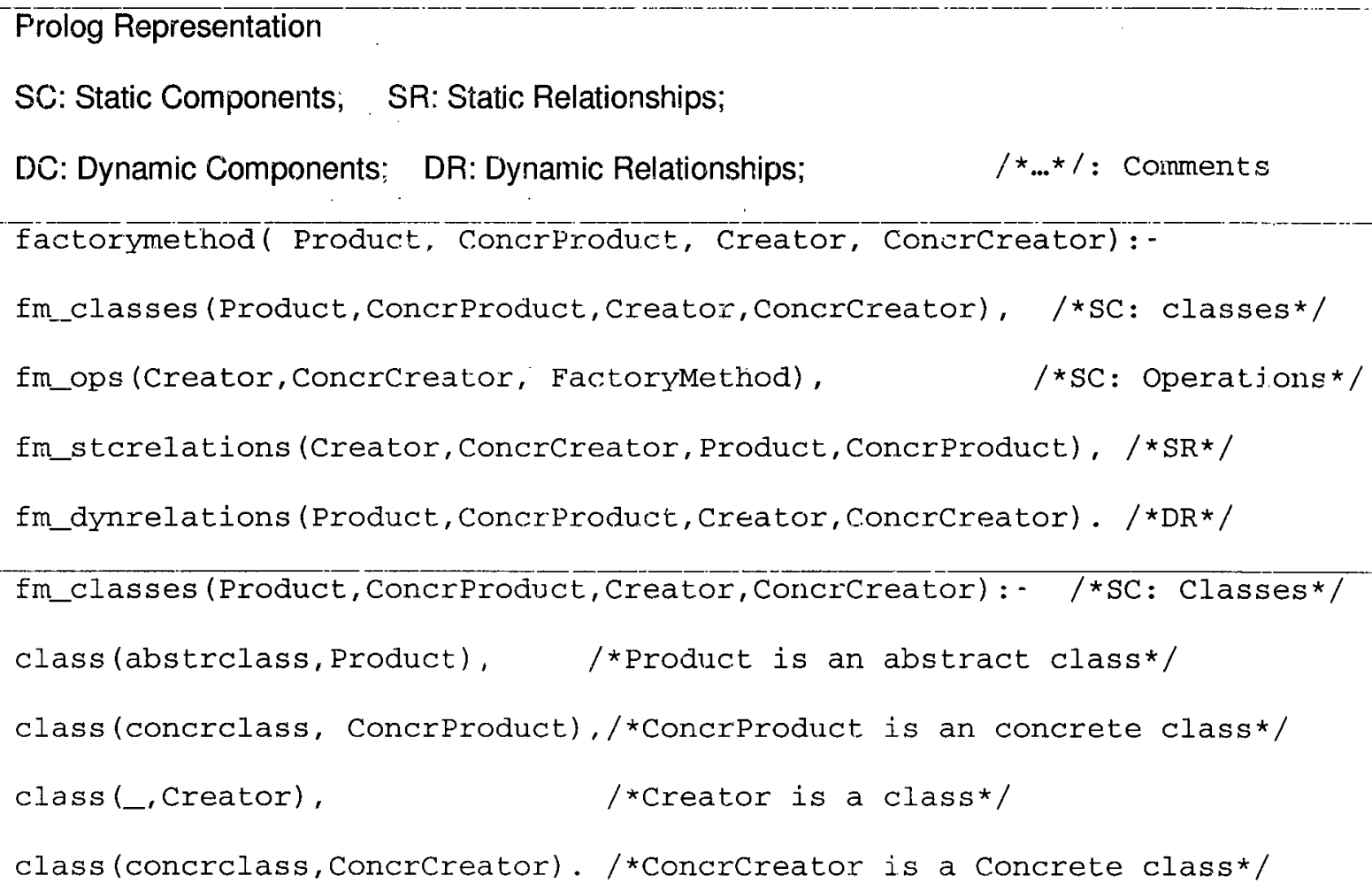




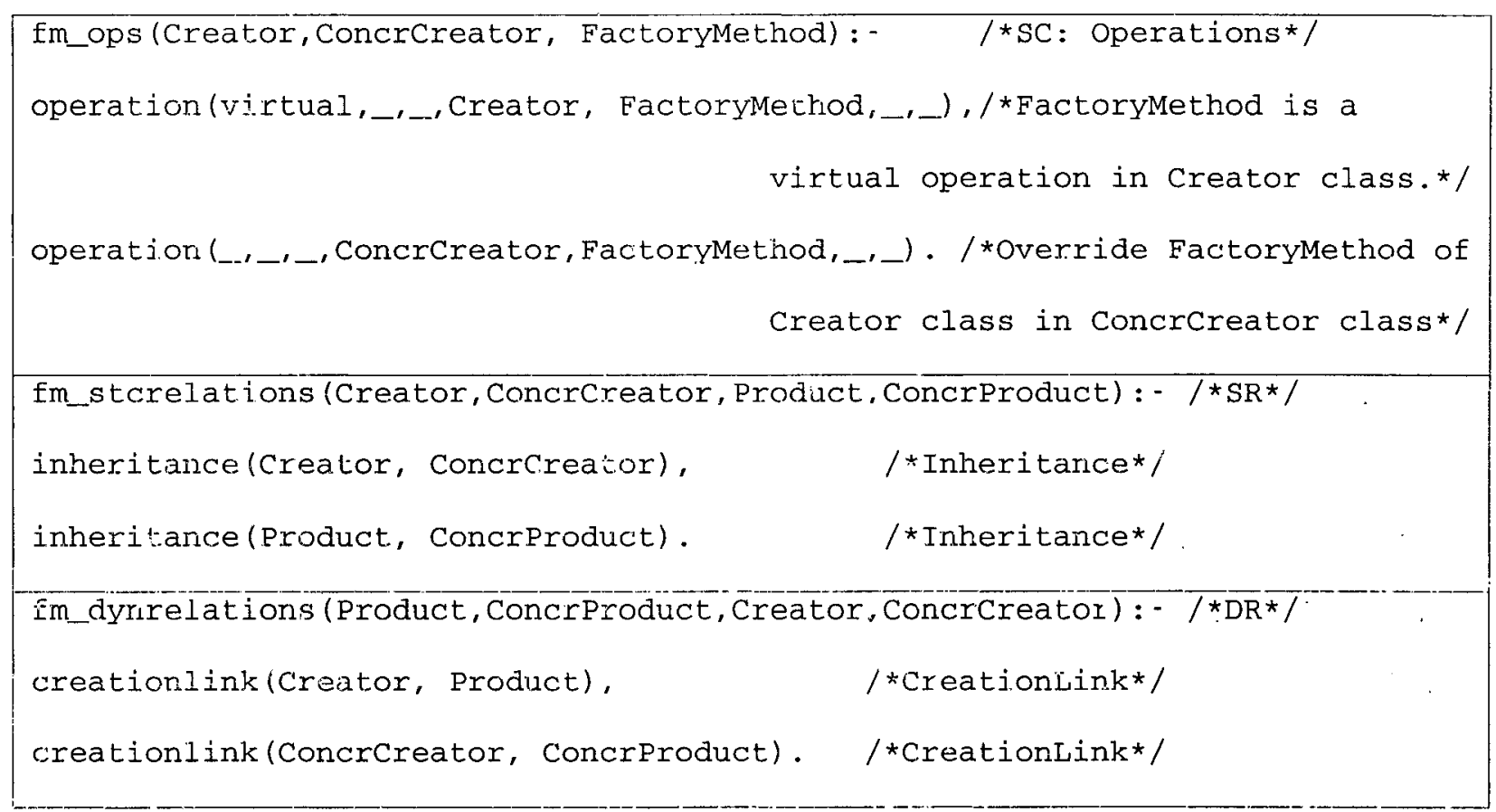

\subsubsection{The Models of Structural Design Patterns}

\subsubsection{Adapter design pattern:}

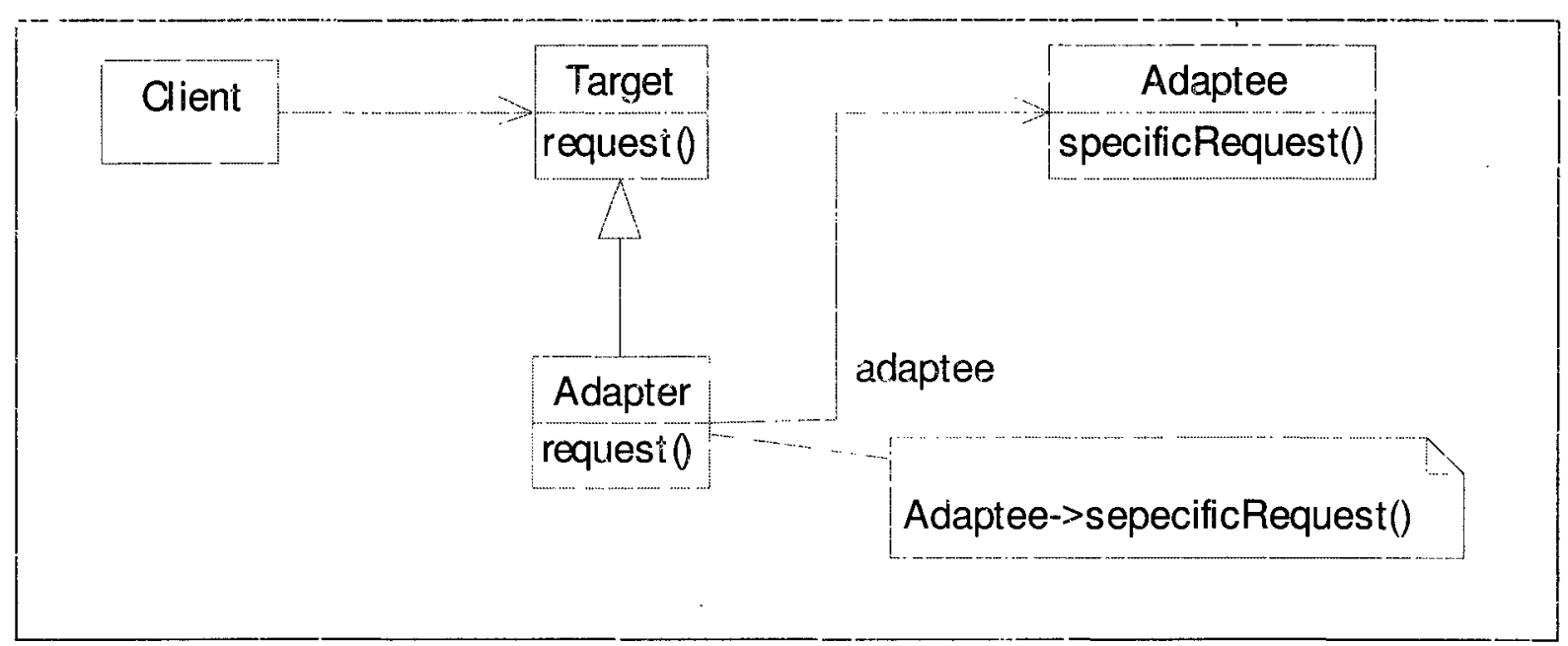

Figure 4-3 UML class diagram of Adapter design patterns 
The Adapter pattern converts the interface of a class into another interface so as to make those classes work together which originally cannot be compatible. When an existing class is expected to use, but its interface cannot match the interface in need, the Adapter pattern can be applied to solve this problem. As shown in Figure 4-3, Class Adapter in the pattern inherits Class Target while it delegates the operation request() from Class Adaptee. These two relationships in the structure of the pattern is the key to identifying the Adapter design pattern and they are reflected well in the Adapter model as shown below.

\subsection{The mapping between the model of Adapter and its Prolog representation}

The mapping between the model of Adapter and its Prolog representation is as follows,

\begin{tabular}{|l|l|}
\hline Elements in the model & Prolog representation \\
\hline Static Components & adapter_classes \\
Classes & adapter_ops \\
Operations & adapter_stcrelations \\
\hline Static Relationships & inheritance \\
Inheritance & delegation \\
Delegation & . \\
\hline
\end{tabular}

Using Figure 4-3 and the table above, the model of the Adapter pattern in Prolog is as follows:

Note: in this table,

SC: Static Components; SR: Static Relationships;

DC: Dynamic Components; DR: Dynamic Relationships; $\quad$ /*..* $\quad$ : Comments

Prolog Representation 


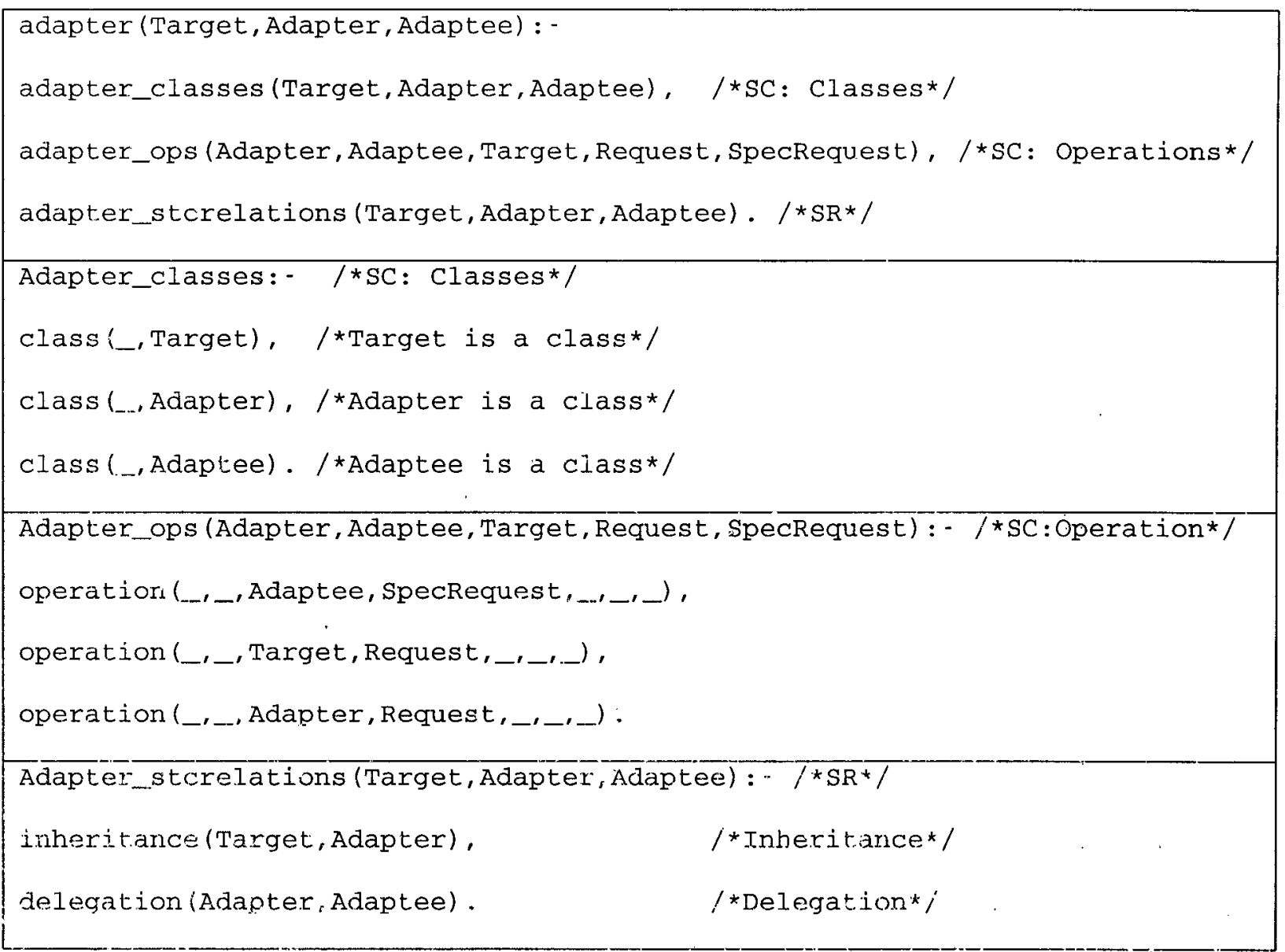

\subsubsection{Composite design pattern:}

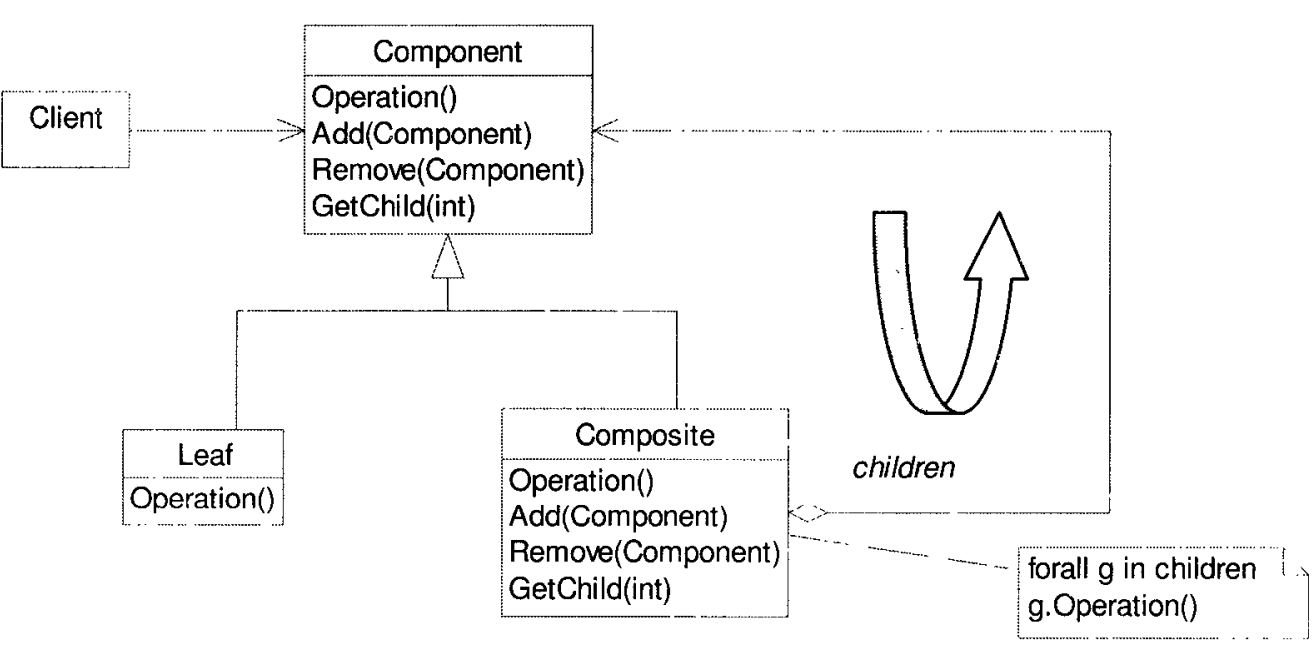

Figure 4-4 UML class diagram of Composite design patterns 
Composite patterns can be used for clients to treat individual objects (components) and their composite (collection) identically so that clients ignore the difference between compositions of objects and individual objects. From Figure 4-4, we can see that there is a loop between Component and Composite. On one hand, Composite inherits from Component; on the other hand Composite aggregates Component. That is, between parent and child classes, Composite pattern requires a part-whole relationship, and this aggregation relationship has a cardinality of one to many. There is a loop existing between a child class and a parent class and this is the key to detecting Composite patterns. When this key is found in class hierarchies it indicates a high possibility of the presence of a Composite pattern. This key has been already included in the model as logic rules,

iriheritarice (Compos, Cpnt)

aggregation (Compos, Cpnt, one-to-many)

\subsection{The mapping between the model of Composite and its Prolog representation}

The mapping between the model of Composite and its Prolog representation is as follows,

\begin{tabular}{|l|l|}
\hline Elements in the model & Prolog representation \\
\hline Static Components & comp_classes \\
Classes & comp_ops \\
Operations & comp_stcrelations \\
\hline Static Relationships & inheritance \\
Inheritance & aggregation \\
Aggregation & delegation \\
Delegation & \\
\hline
\end{tabular}


The model of the Composite Design pattern in Prolog is as follows:

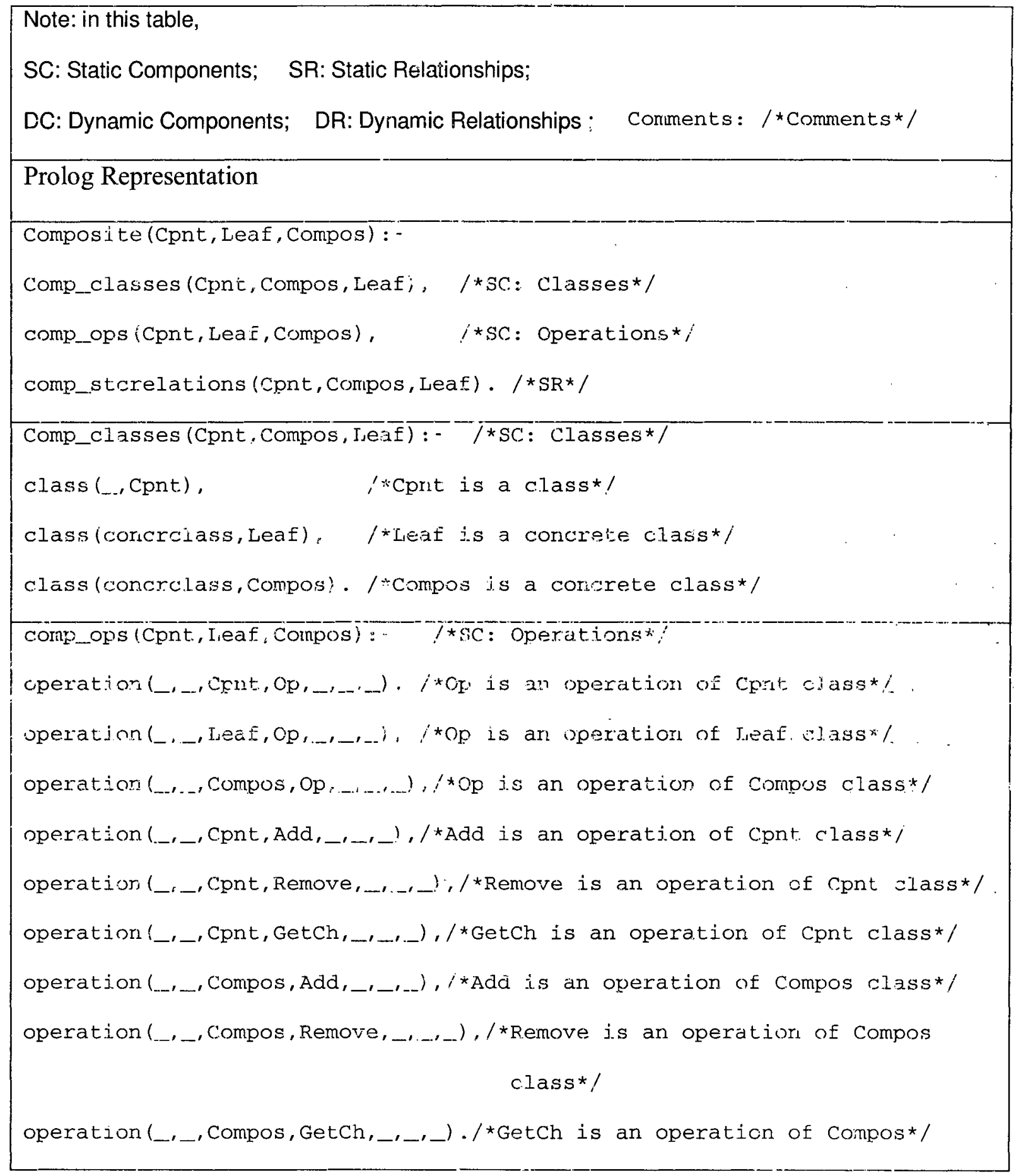




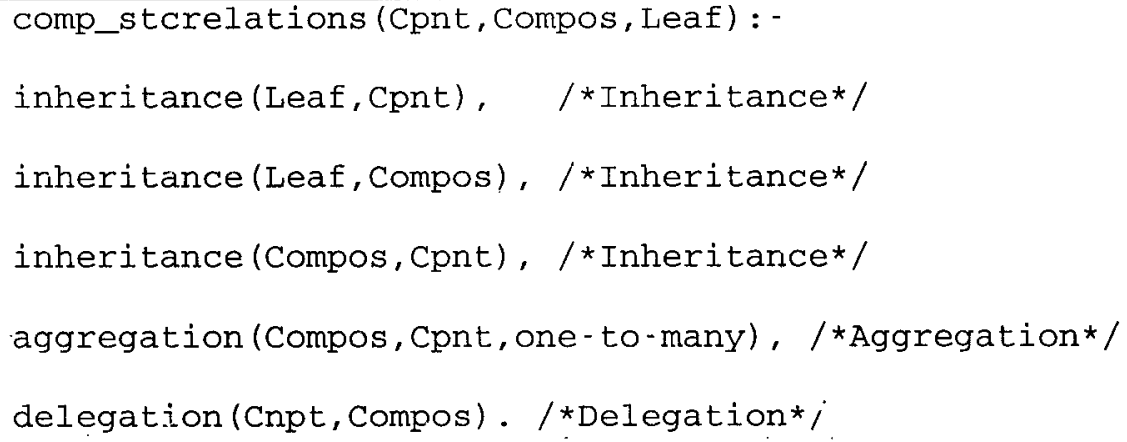

\subsubsection{Decorator Design Pattern:}

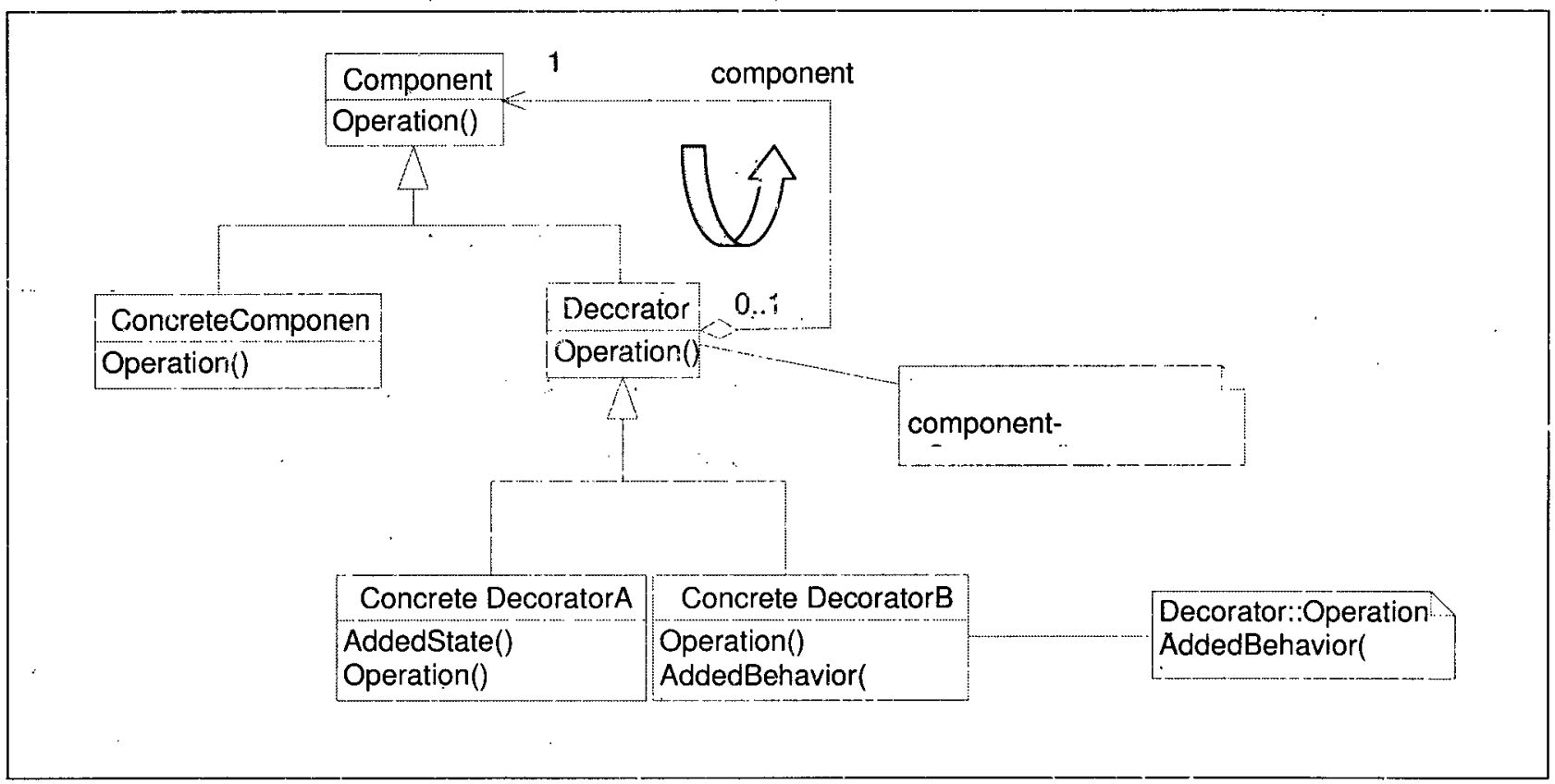

Figure 4-5 UML class Diagram of $\overline{\text { Decorator design patterns }}$

The Decorator pattern is used to dynamically attach additional responsibilities to an object. Decorators provide a flexible alternative to subclassing for extending functionality. As shown in Figure 4-5 the basic structure is similar to that of the Composite pattern, but the difference between the Decorator pattern and the Composite pattern is seen in the aggregation relationship between the child class and the parent 
class. In the Composite pattern the cardinality in the aggregation between Component class and Composite class is one to many, but the aggregation between Component class and Decorator class in the Decorator pattern is one to one. This difference in their structure can be used to identify the Decorator patterns in a code.

\subsection{The mapping between the model of Decorator and its Prolog representation}

The mapping between the model of Decorator and its Prolog representation is as follows,

\begin{tabular}{|l|l|}
\hline Elements in the model & Prolog representation \\
\hline Static Components & dec_classes \\
Classes & dec_ops \\
Operations & dec_stcrelations \\
\hline Static Relationships & inheritance \\
Inheritance & aggregation \\
Aggregation & delegation \\
\hline Delegation & \\
\hline
\end{tabular}

Using Figure 4-5 and the table above, the model of the Decorator Design Pattern in Prolog is as follows:

Note: in this table,

SC: Static Components; SR: Static Relationships;

DC: Dynamic Components; DR: Dynamic Relationships; $\quad$ /*..*/: Comments

Prolog Representation

decorator (Cpnt, ConcrCpnt, Deco, ConcrDecoA, ConcrDecoB) :-

dec_classes (Cpnt, ConcrCpnt, Deco,ConcrDecoA, ConcrDecoB) , /*SC: Classes*/

dec_ops (Cpnt, ConcrCpnt, Deco,ConcrDeCOA,ConcrDecoB), /*SC: Operations*/

dec_stcrelations (Cpnt, ConcrCpnt, Deco,ConcrDecoA, ConcrDecoB). $/ * S R * /$ 


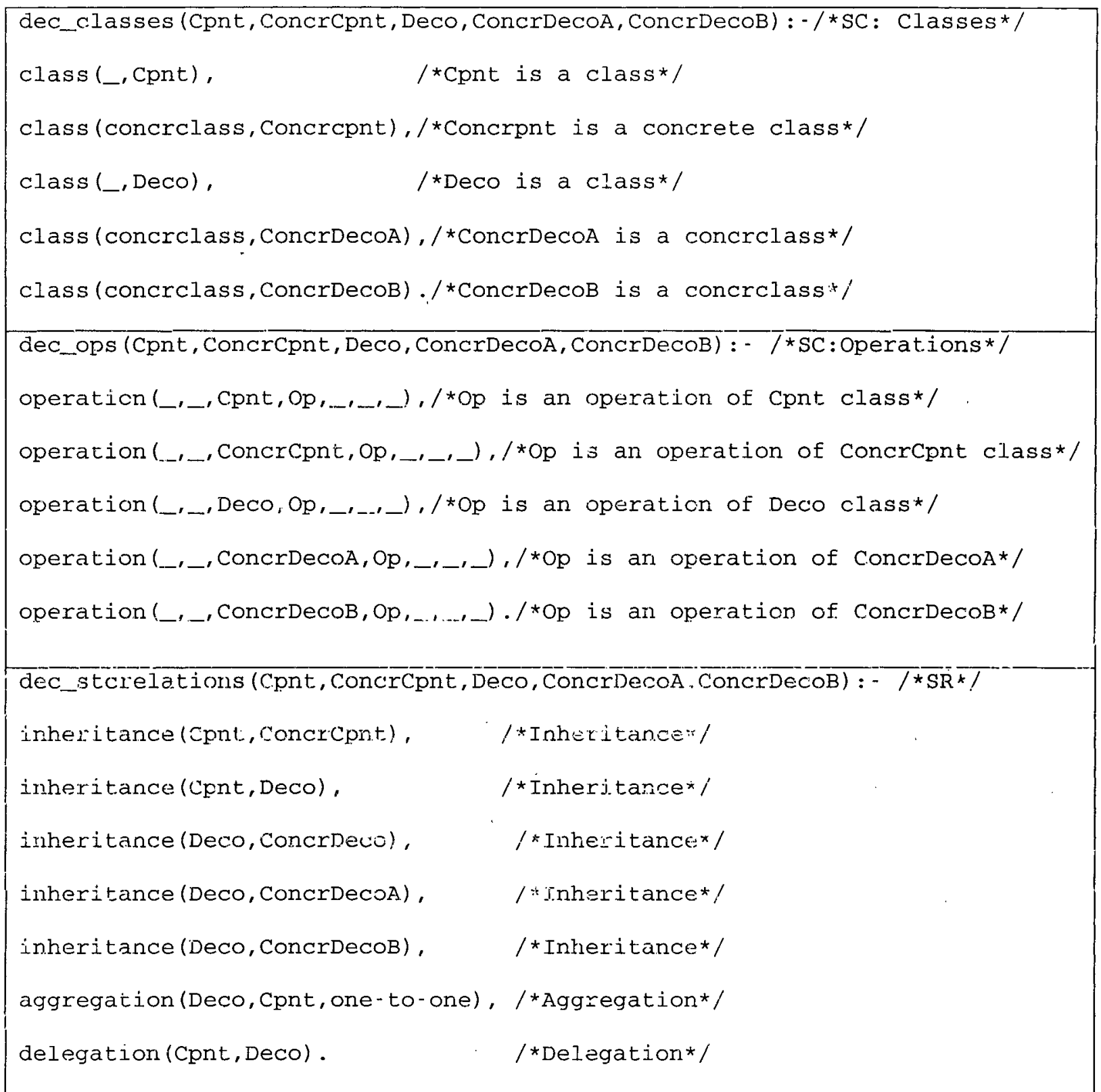

\subsubsection{Proxy design pattern:}




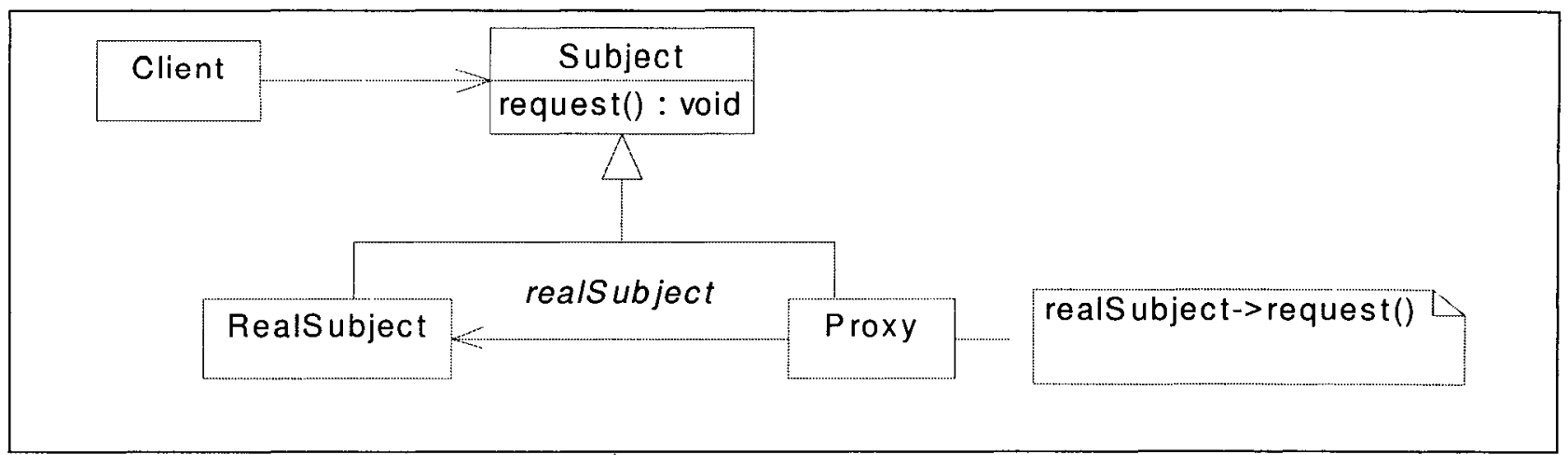

Figure 4-6 UML class diagram of Proxy design patterns

The Proxy design pattern is used as a surrogate for another object in order to control access to it. The reason why a Proxy is used to control access to an object is to defer the full cost of its creation and initialization until it is actually needed. The key to identifying the Proxy design pattern is Delegation relationship between Proxy and RealSubject; Proxy is the delegator while RealSubject is delegatee. It is reflected in the model as follows:

delegation (RealSubj, Proxy).

\subsection{The mapping between the model of Proxy and its Prolog representation}

The mapping between the model of Proxy and its Prolog representation is as follows:

\begin{tabular}{|l|l|}
\hline Elements in the model & Prolog representation \\
\hline Static Components & proxy_classes \\
Classes & proxy_ops \\
Operations & proxy_stcrelations \\
\hline Static Relationships & inheritance \\
Inheritance & delegation \\
Delegation & \\
\hline
\end{tabular}

Using Figure 4-6 and the table above, the model of the Proxy design pattern is as follows: 


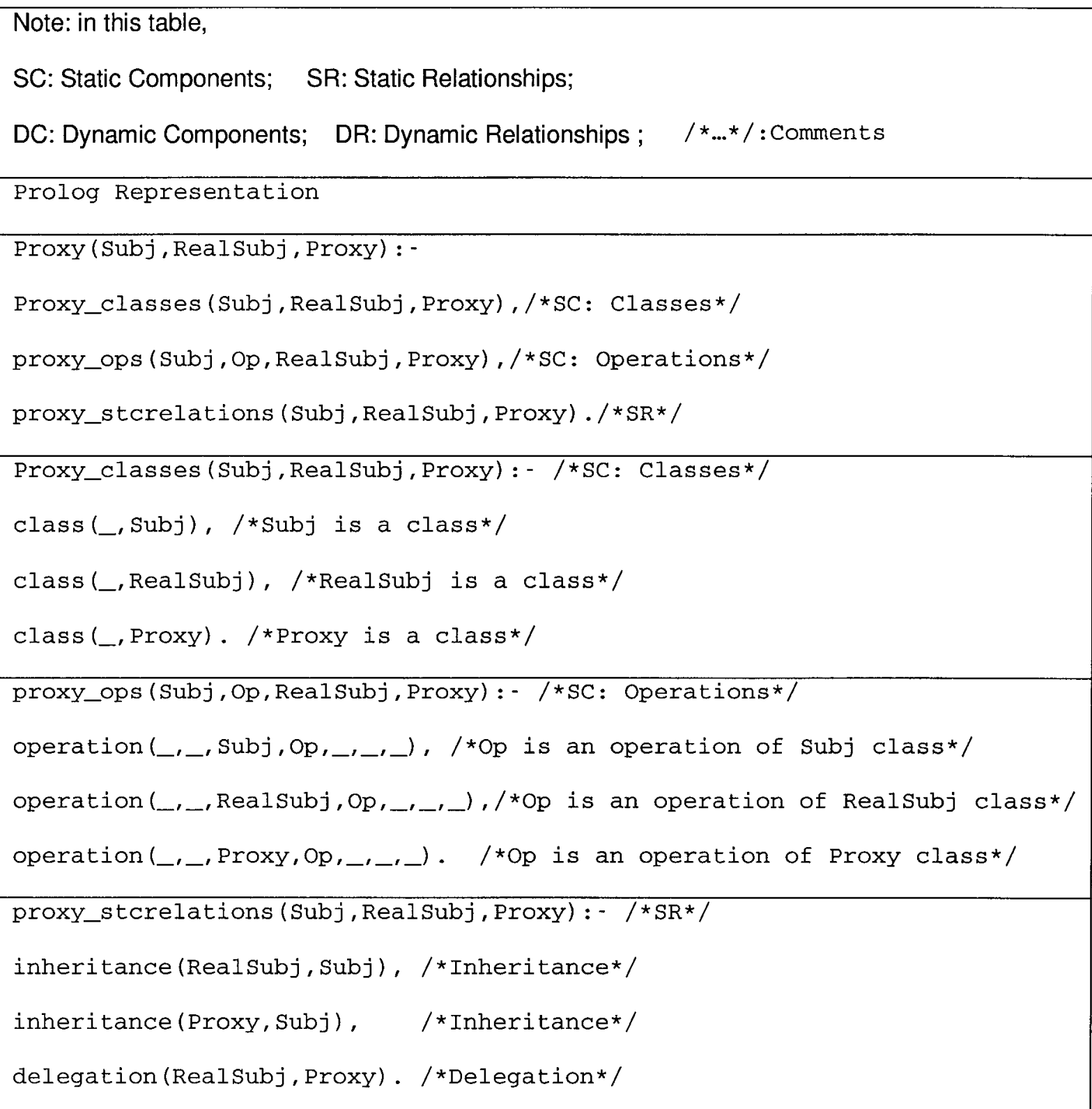

\subsubsection{Bridge design pattern:}

Bridge design pattern bridges the abstraction and its implementation and let them vary independently. In doing so we can avoid a permanent binding between an abstraction and its implementation. The key to identifying Bridge design pattern is the delegation relationship between Abstraction and Implementor as well as inheritance in class hierarchy. 


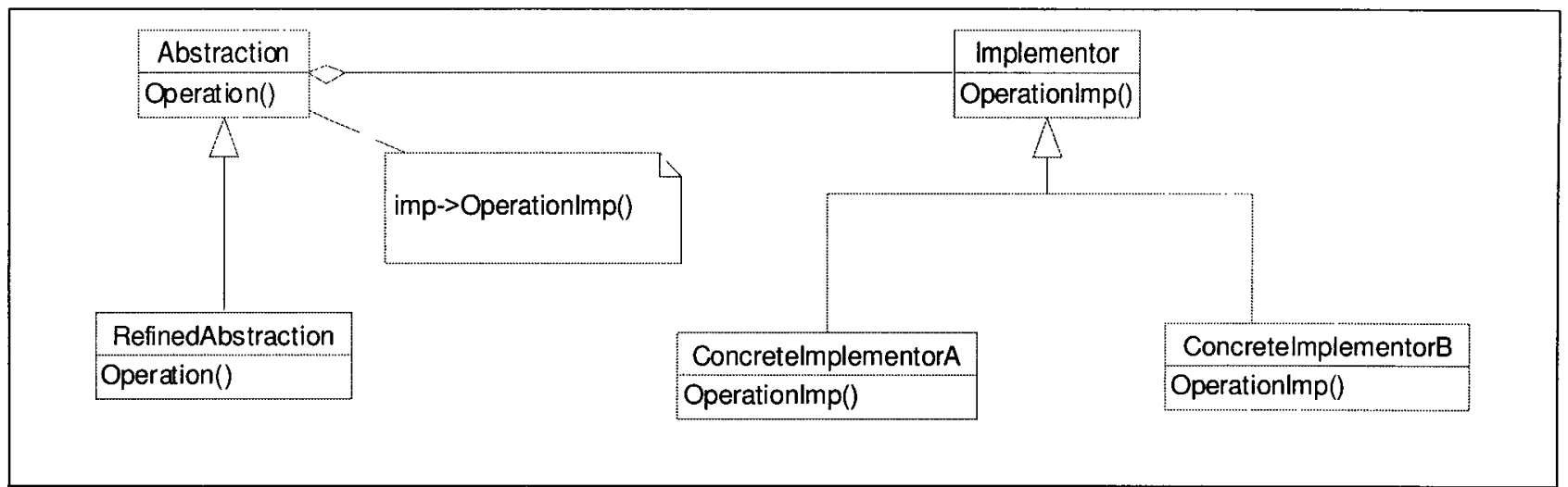

Figure 4-7 UML class diagram of Bridge design patterns

\subsection{The mapping between the model of Bridge and its Prolog representation}

The mapping between the model of Bridge and its Prolog representation is as follows:

\begin{tabular}{|l|l|}
\hline Elements in the model & Prolog representation \\
\hline Static Components & bridge_classes \\
Classes & bridge_ops \\
\hline Static Relationships & bridge_stcrelations \\
Inheritance & inheritance \\
Delegation & delegation \\
\hline
\end{tabular}

Using Figure 4-7 and the table above, the model of the Bridge design pattern in Prolog is as follows:

\begin{tabular}{l} 
Note: in this table, \\
SC: Static Components; SR: Static Relationships; \\
DC: Dynamic Components; DR: Dynamic Relationships; $\quad / * \ldots * /:$ Comments \\
\hline Prolog Representation
\end{tabular}




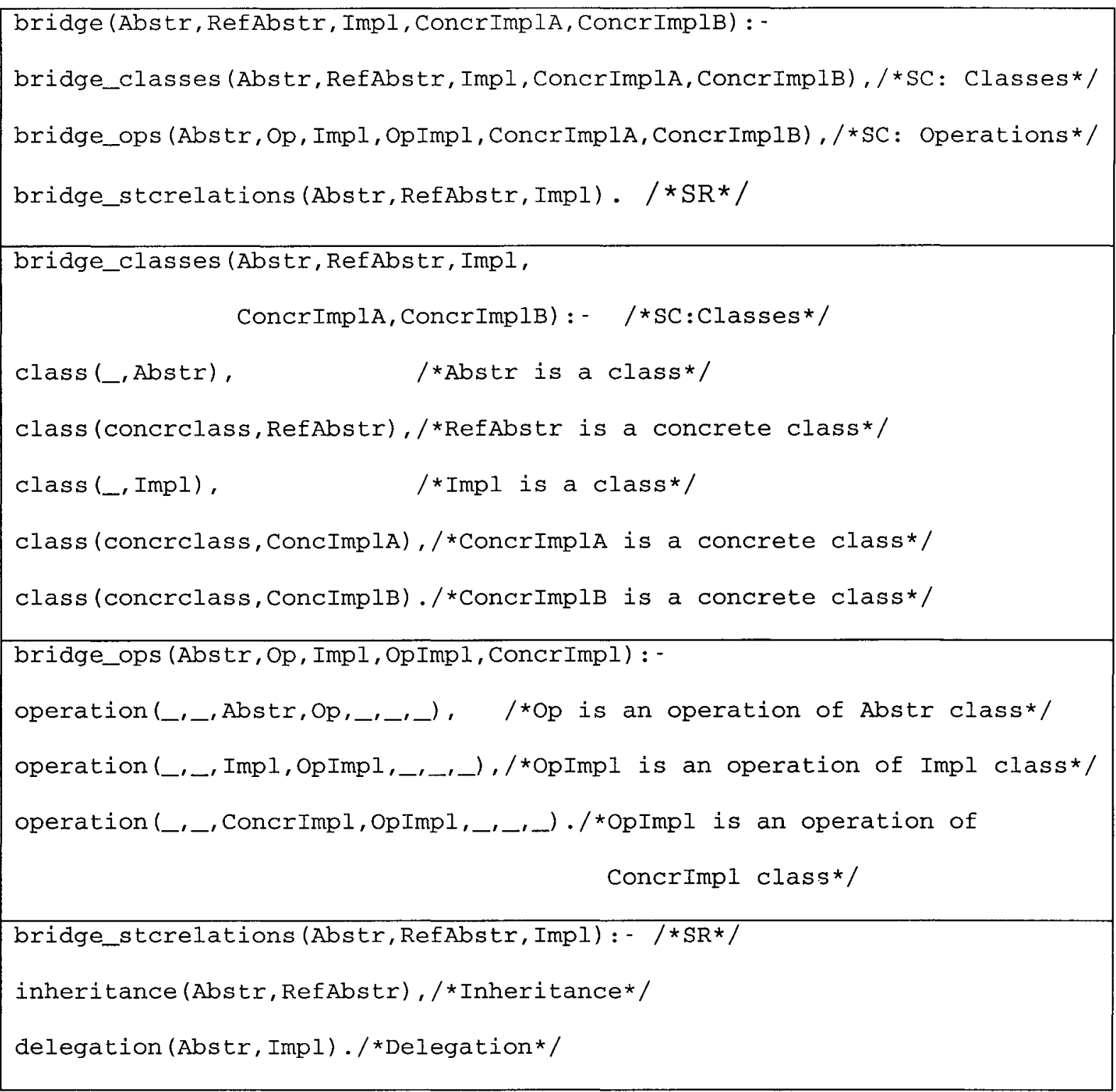

\subsubsection{The Models of Behavioral Design Pattems}

\subsubsection{Strategy Design Pattern:}

The strategy design pattern provides a common interface, a strategy class to encapsulate a group of algorithms so that they can be varied independently from clients that uses it. As shown in Figure 4-8, each algorithm can be varied independently of its context, this make the Strategy class easer to switch, understand, and extend. 


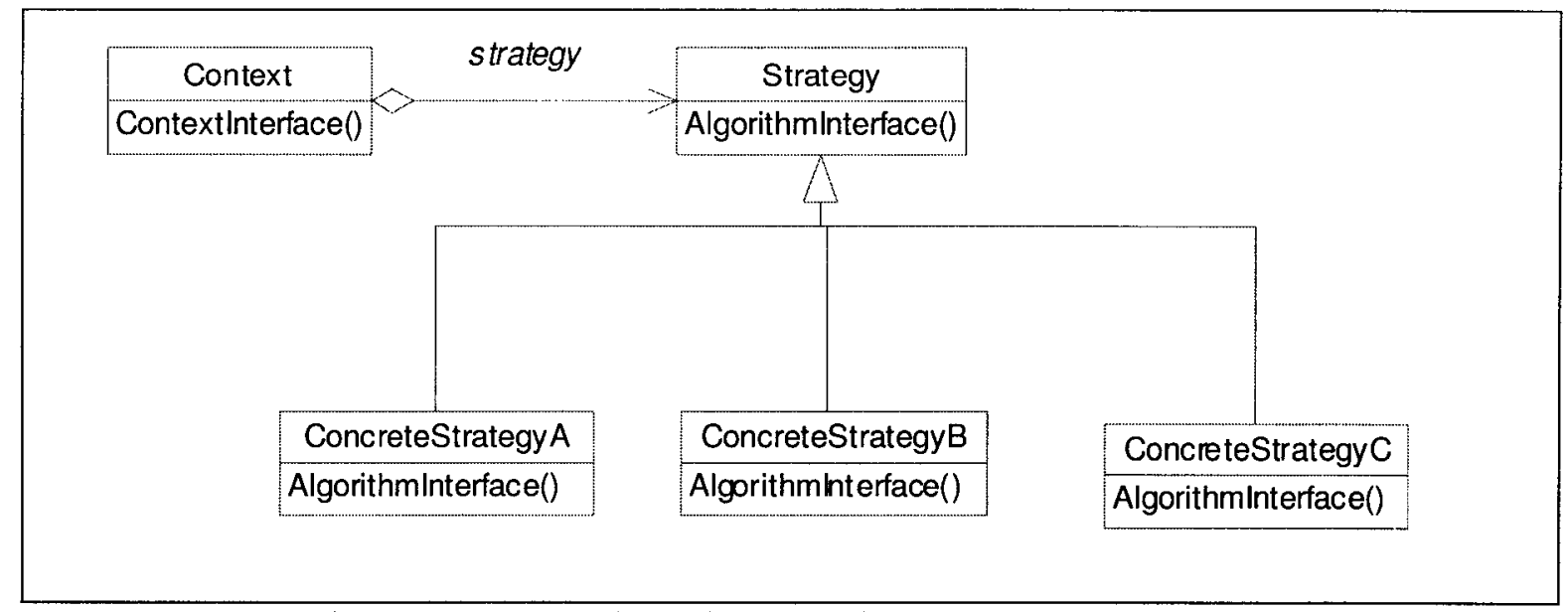

Figure 4-8 UML class diagram of Strategy design patterns

The key to identifying Strategy design patterns is the aggregation relationships between context and strategy and the inheritance relationships between Strategy and ConcreteStrategy, as well as AlgorithmInterface overridden by each subclass of Strategy. This key is reflected in Operation of Static Component and the Static Relationships in the model below.

\subsection{The mapping between the model of Strategy and its Prolog representation}

The mapping between the model of Strategy and its Prolog representation is as follows:

\begin{tabular}{|l|l|}
\hline Elements in the model & Prolog representation \\
\hline Static Components & \\
Classes & str_classes \\
Operations & srt_ops \\
\hline Static Relationships & str_stcrelations \\
Inheritance & inheritance \\
\hline
\end{tabular}

Using Figure 4-8 and the table above, the model of the Strategy design pattern in Prolog is as follows:

Note: in this table,

SC: Static Components; SR: Static Relationships; 


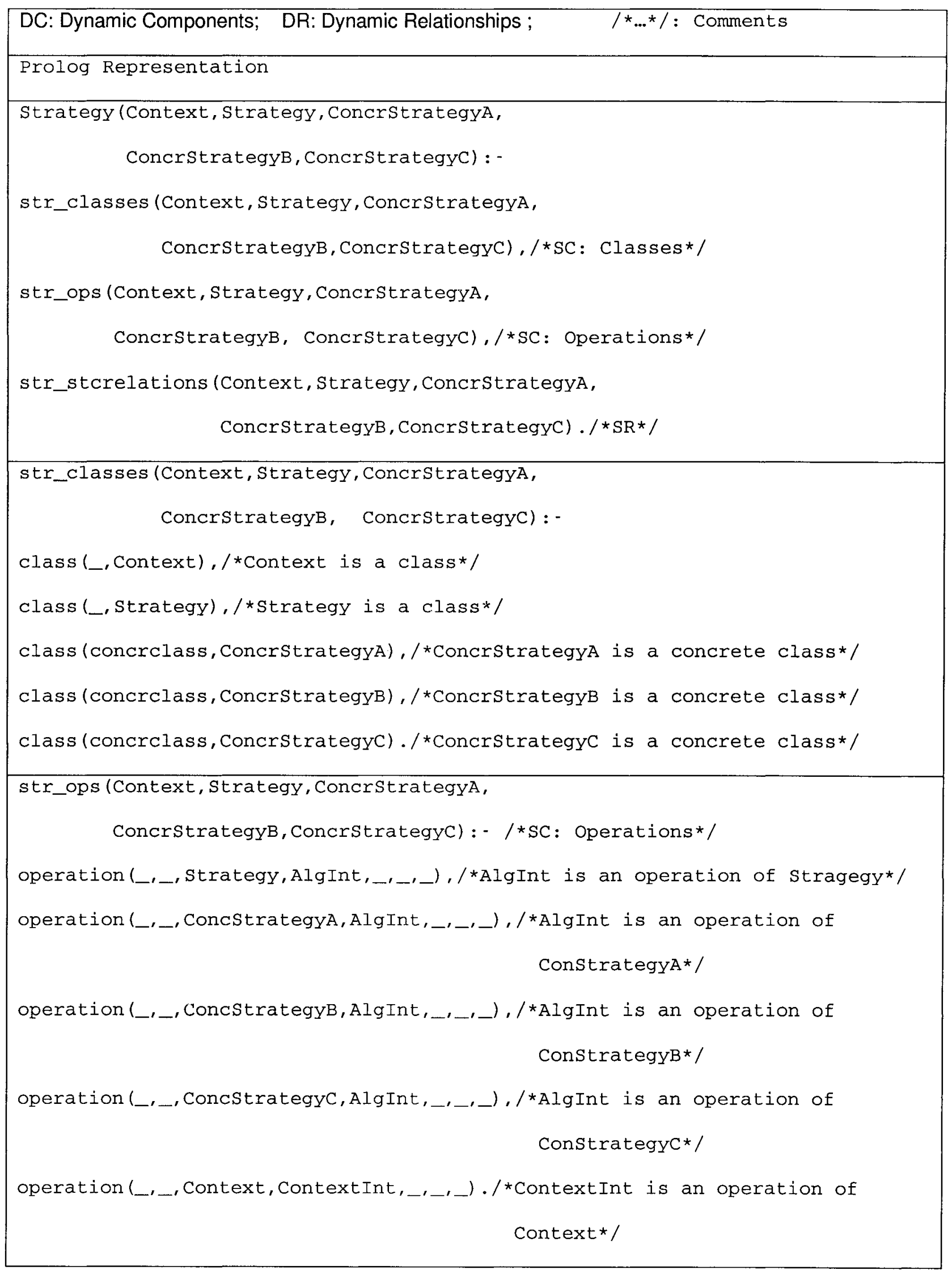




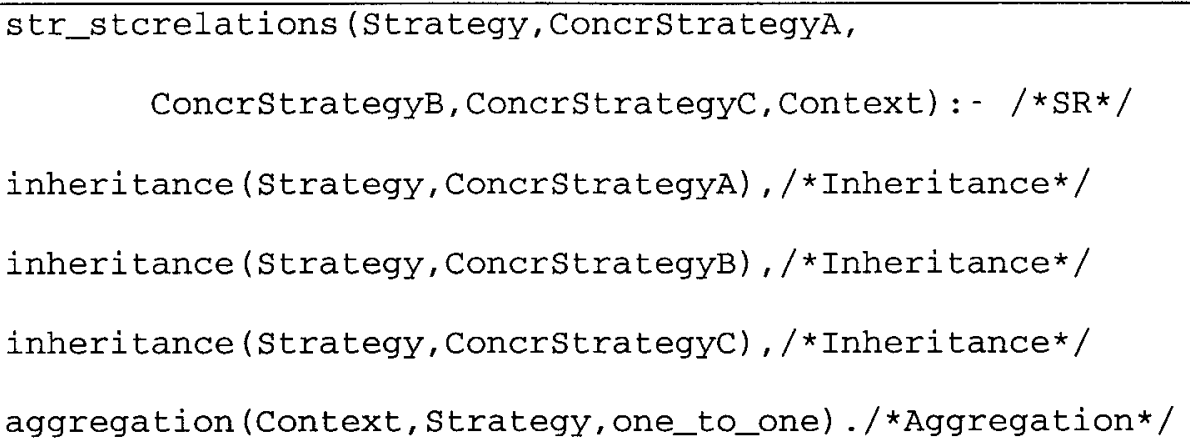

Though the Strategy design pattern belongs to the Behavioral design pattern its dynamic characteristics are too vague to be used as an identification of this pattern. So the static structure of it becomes our choice instead of dynamic behavior.

\subsubsection{Observer design pattern}

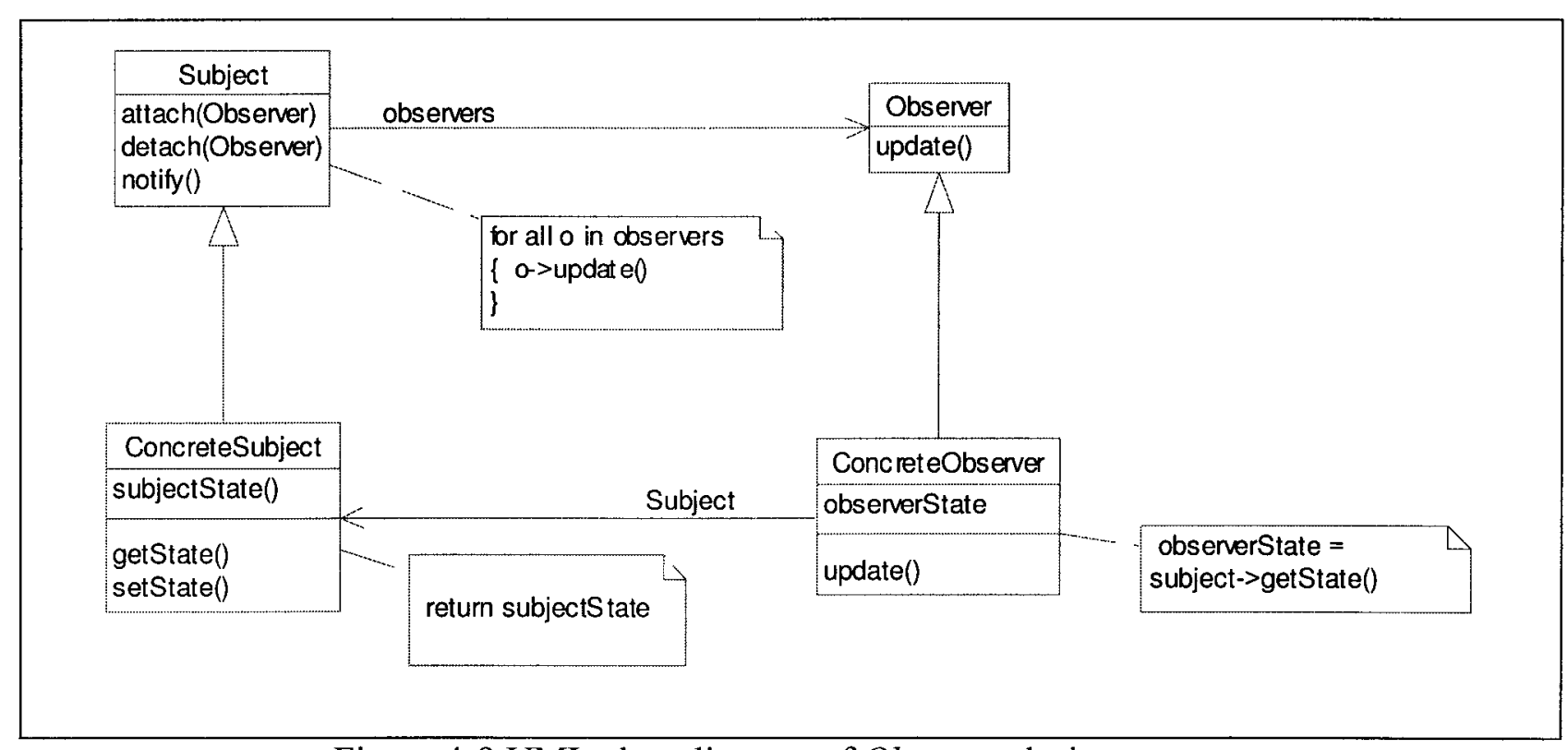

Figure 4-9 UML class diagram of Observer design patterns

The Observer design pattern defines a one-to-many dependency between objects so that when one object changes state, all its dependents are notified and updated automatically. 
Here we provide two kinds of models for Observer design patterns, one is based on static characteristics (i.e., structure) of the pattern and the other is based on dynamic characteristics (i.e. behavior) of the pattern.

\subsection{The mapping between the structure-based model of Observer and its Prolog representation}

The mapping between the structure-based model of Observer and its Prolog representation is as follows:

\begin{tabular}{|l|l|}
\hline Elements in the model & Prolog representation \\
\hline Static Components & obs_classes \\
Classes & obs_ops \\
Operations & obs_stcrelations \\
\hline Static Relationships & inheritance \\
\hline
\end{tabular}

- Using Figure 4-9 and the table above, the structure-based model of the Observer design pattern only in terms of static information:

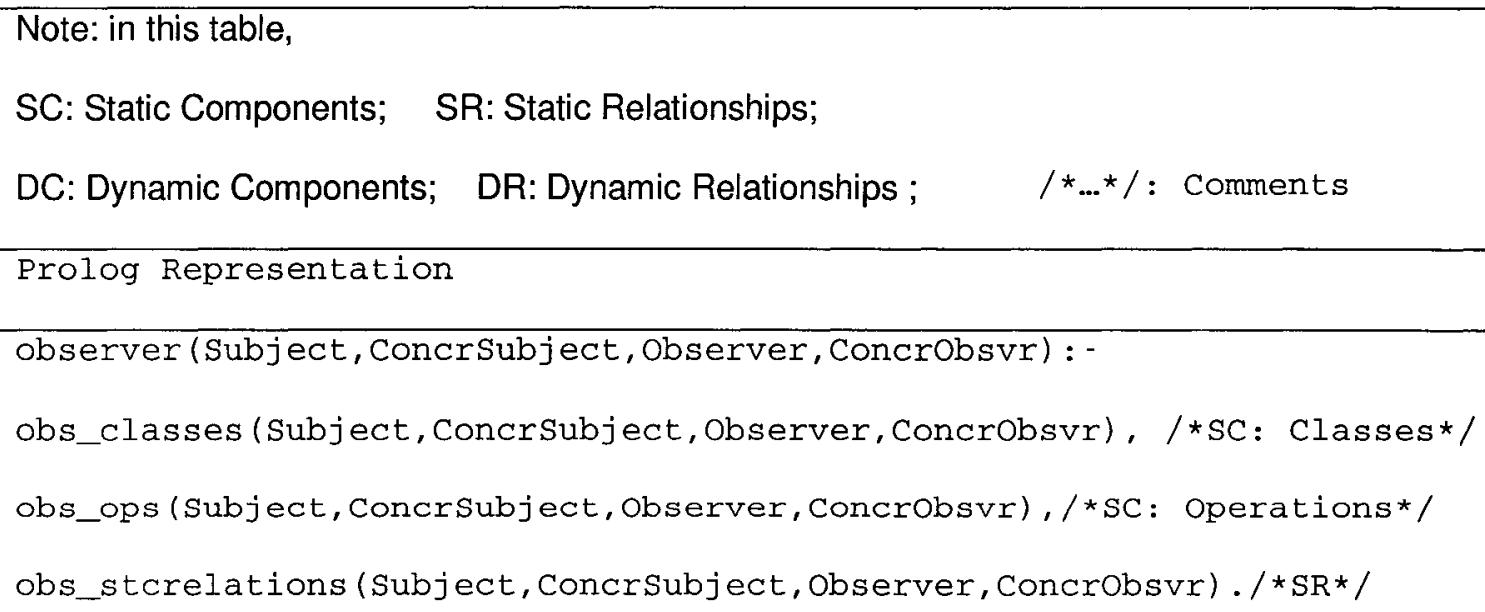




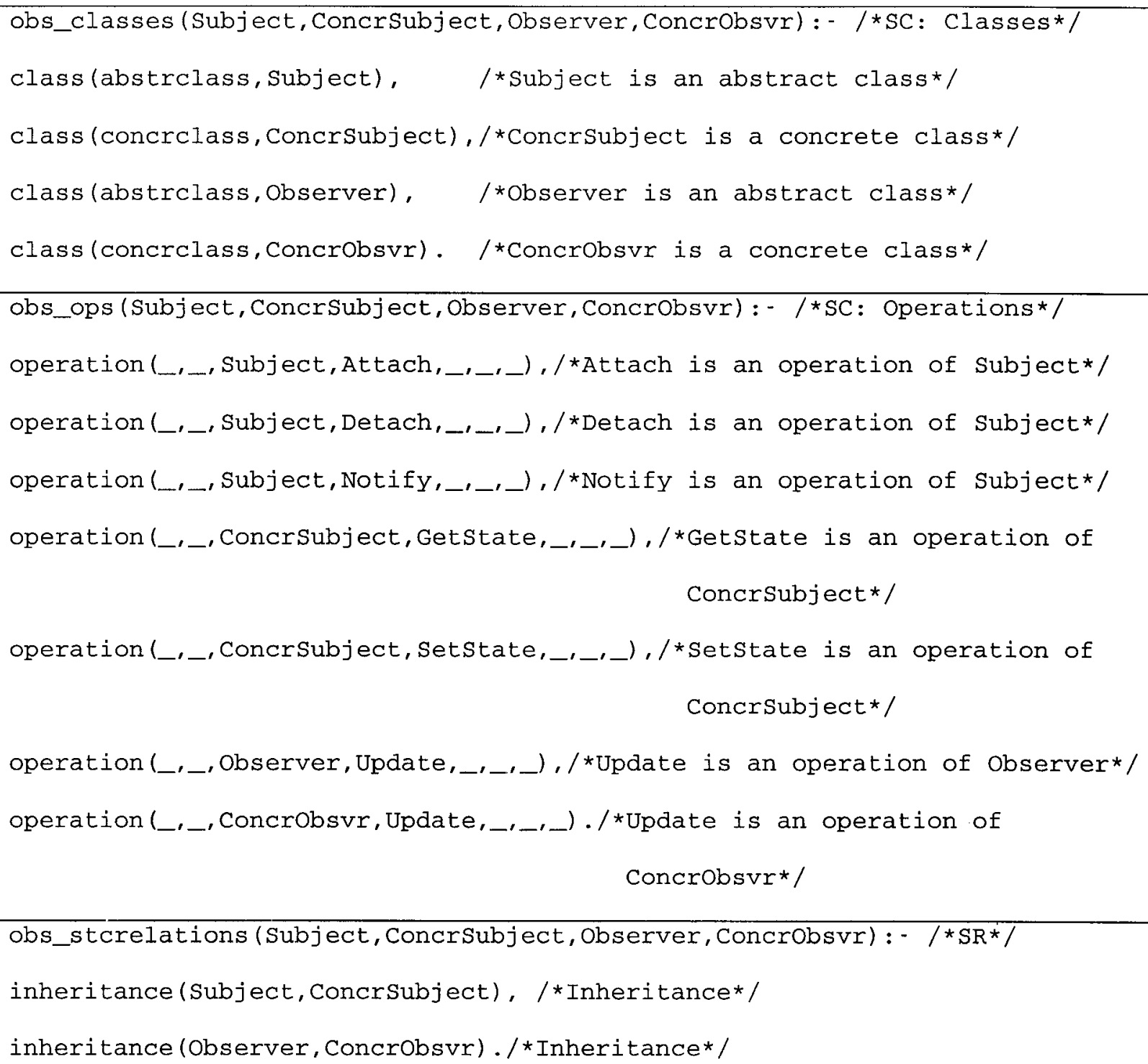

- The Behavioral-based model of Observer design patterns in Prolog 


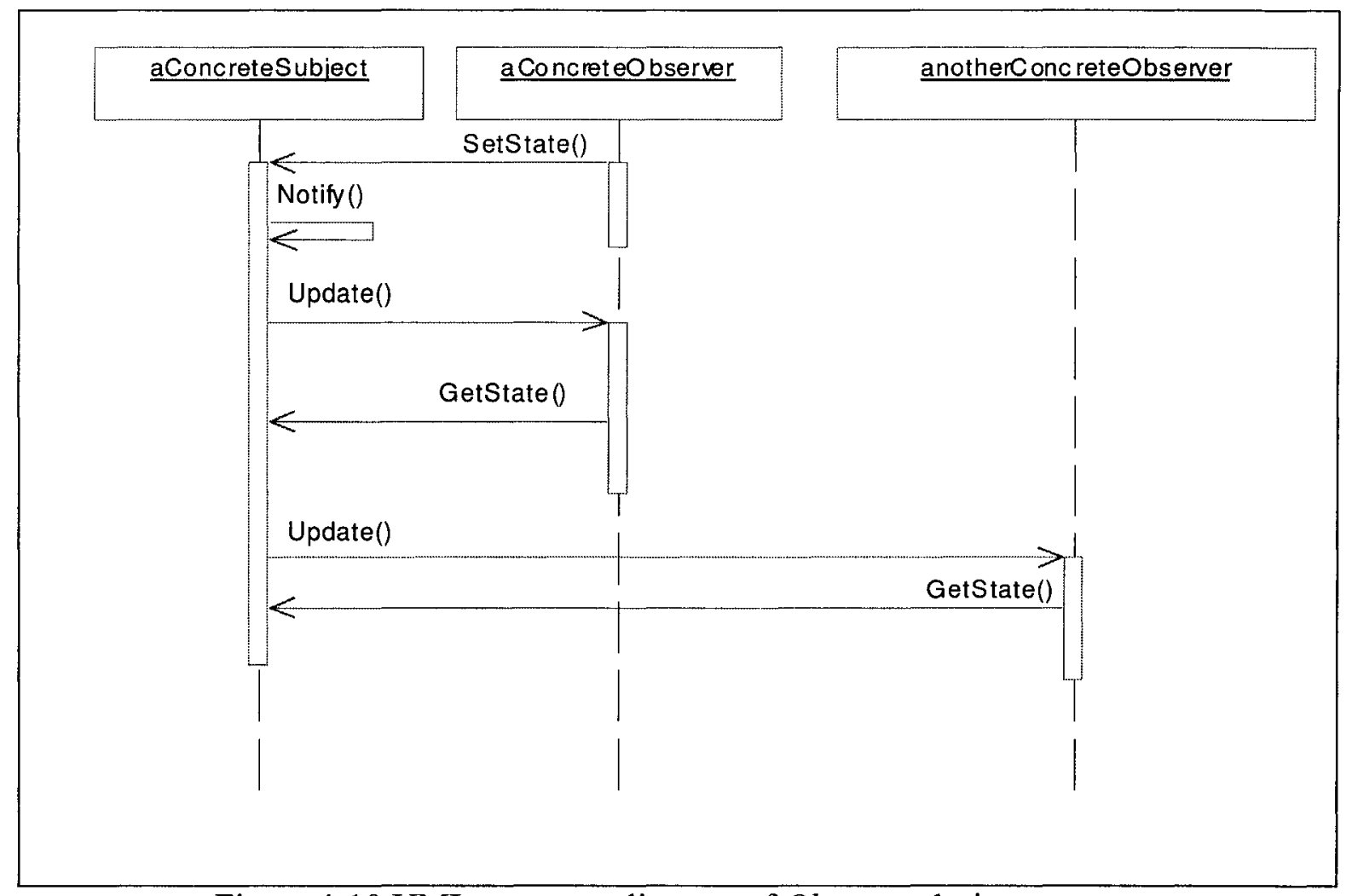

Figure 4-10 UML sequence diagram of Observer design patterns

\subsection{The mapping between the behavioral-based model of Observer and its}

\section{Prolog representation}

The mapping between the behavioral-based model of Observer and its Prolog representation is as follows,

\begin{tabular}{|l|l|}
\hline Elements in the model & Prolog representation \\
\hline Dynamic Components & obs_dyncomps \\
object & $\begin{array}{l}\text { obs_dynrelations } \\
\text { sendmessage }\end{array}$ \\
Dynamic Relationships & selfmessage \\
SelfMessage & \\
\hline
\end{tabular}


The following is the alternative behavioral-based model of the Observer design pattern:

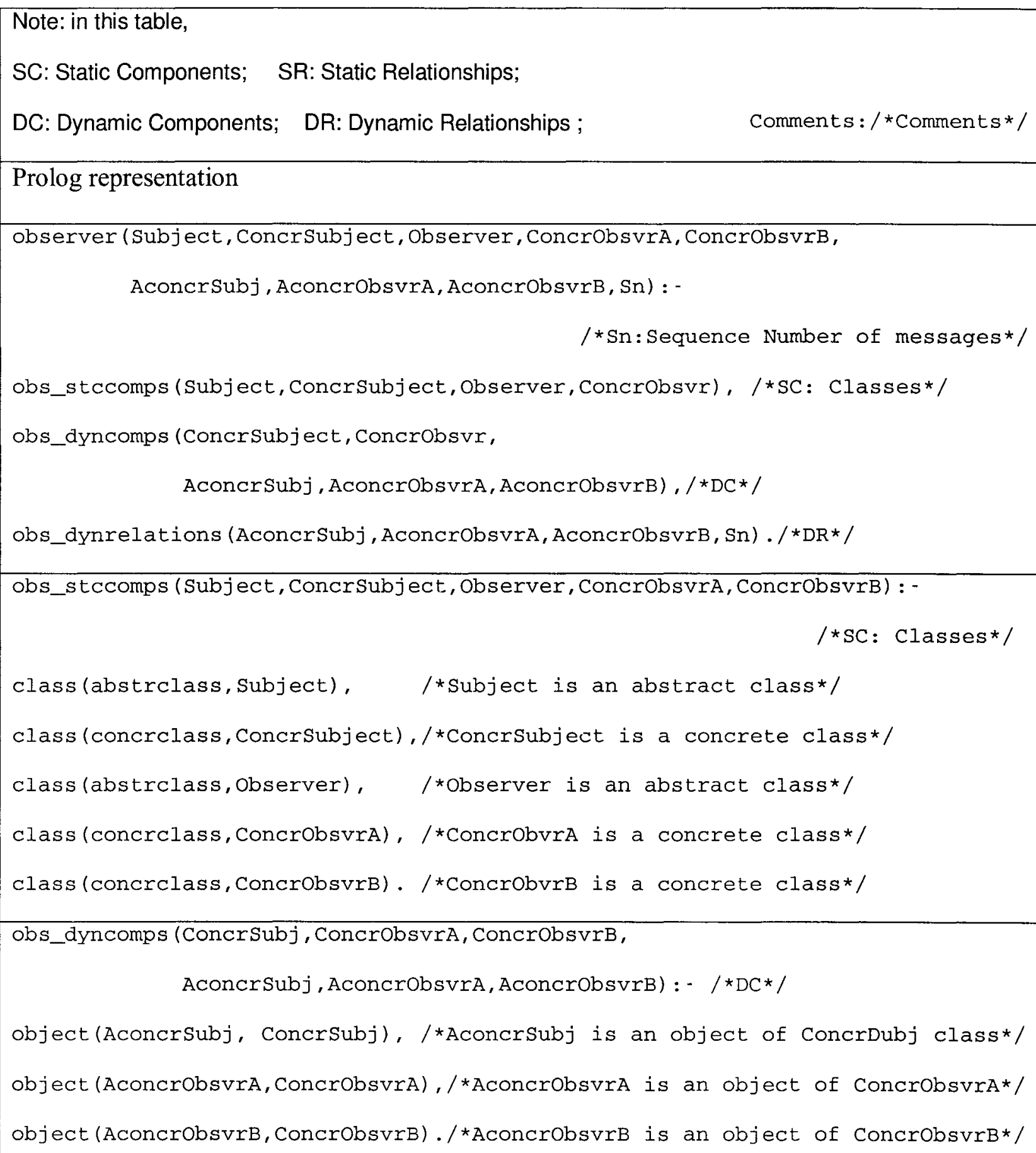




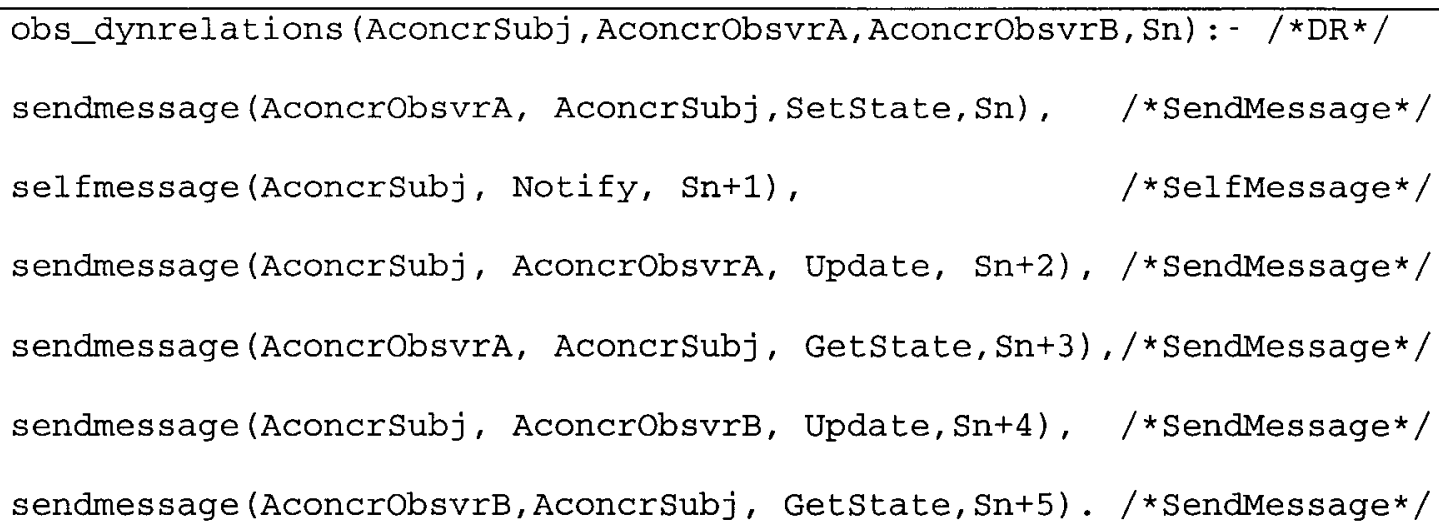

Observer design patterns are of strong dynamic characteristics. Suppose that dynamic information such as SendMessage and Object is available, it would be easy to identify Observer design patterns with high precision.

In contrast, in the aspect of static components and relationships of an Observer pattern there are only two class hierarchies that are key to identifying the pattern that would result in many false instances of the pattern. In our case study, after the structuralbased model is used to identify Observer design patterns, many false instances were generated. This is because in the Observer pattern the structural characteristics that can be used as the key for identifying is not so strong that a set of classes that have the same class hierarchies can have different behavior.

\subsubsection{Chain of Responsibility}

Chain of Responsibility aims to "avoid coupling between the sender of a request and its receiver by giving more than one object a chance to handle the request."[Gam95]. It makes use of some mechanisms like polymorphism to form a chain among objects. A request from senders will be passed along the chain until an object handles it.

As shown in Figure 4-11 it has strong dynamic characteristics. When a message is exchanged by several objects one by one it implies that a chain is formed. By finding 
such a chain in related dynamic information Chain of Responsibility design patterns can be identified easily.

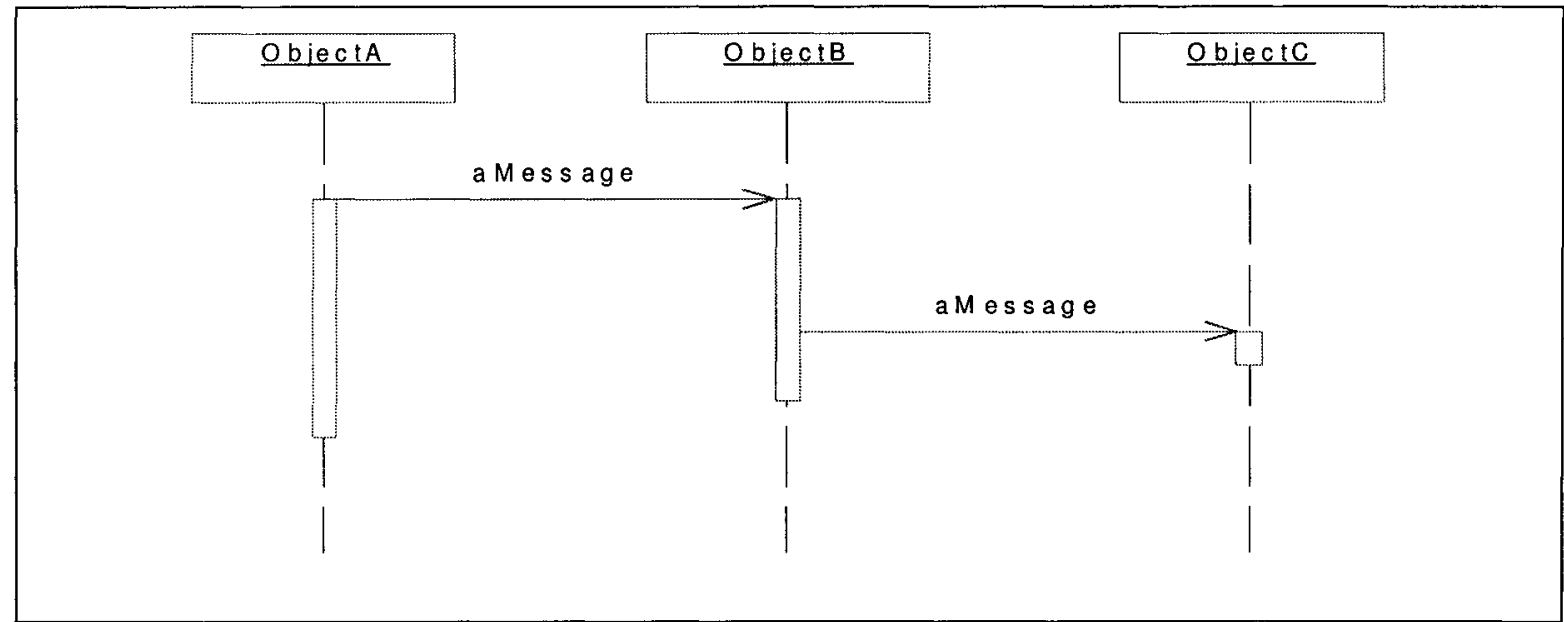

Figure 4-11 UML sequence diagram of Chain of Responsibility design patterns

Here is the model based on dynamic characteristics called behavioral-based model.

\subsection{The mapping between the model of Chain of Responsibility and its Prolog representation}

The mapping between the model of Chain of Responsibility and its Prolog representation is as follows:

\begin{tabular}{|l|l|}
\hline Elements in the model & Prolog representation \\
\hline Dynamic Components & cor_dyncomps \\
Object & object \\
\hline Dynamic Relationships & cor-dynrelations \\
SendMessage & sendmessage \\
\hline
\end{tabular}

Using Figure 4-11 and the table above, the behavioral-based model of Chain of Responsibility in Prolog is as follows: 


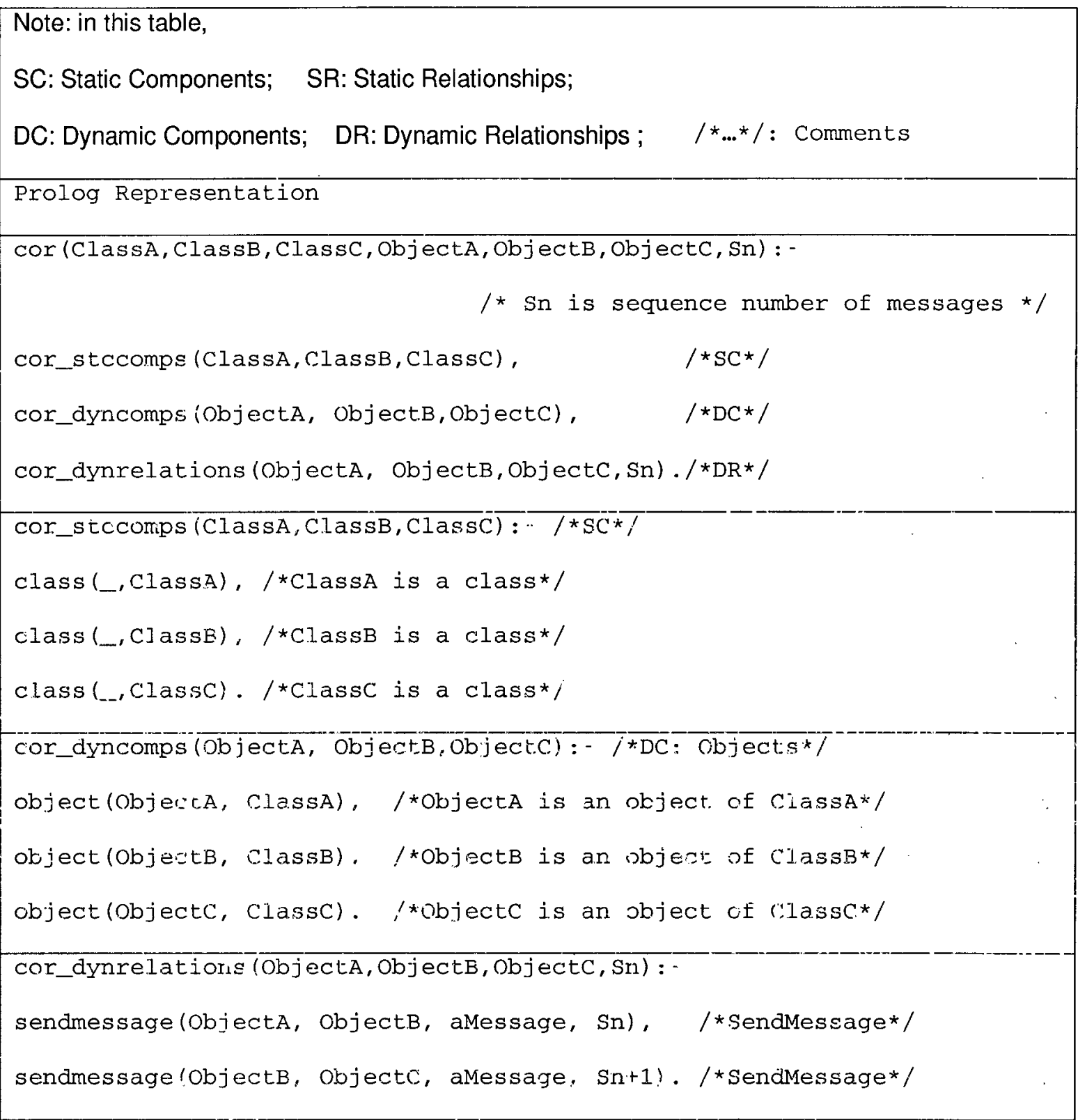

The Chain of Responsibility pattern has strong dynamic characteristics; the soul of this pattern is its behavior rather than its structure. If this pattern is identified merely based on static information it is likely that some implementation variants of the pattern are lost. For example, in [Gam95], the chain is formed by polymorphism, but in [Coo98], it is implemented by directly defining a Chain interface explicitly responsible for passing 
the message among objects. The structure of both is different but their behavior is the same.

\subsubsection{Visitor}

Visitor represents an operation to be performed on the elements of an objects structure. Visitor design pattern allows the definition of a new operation without changing the classes of the elements on which it operates [Gam95].

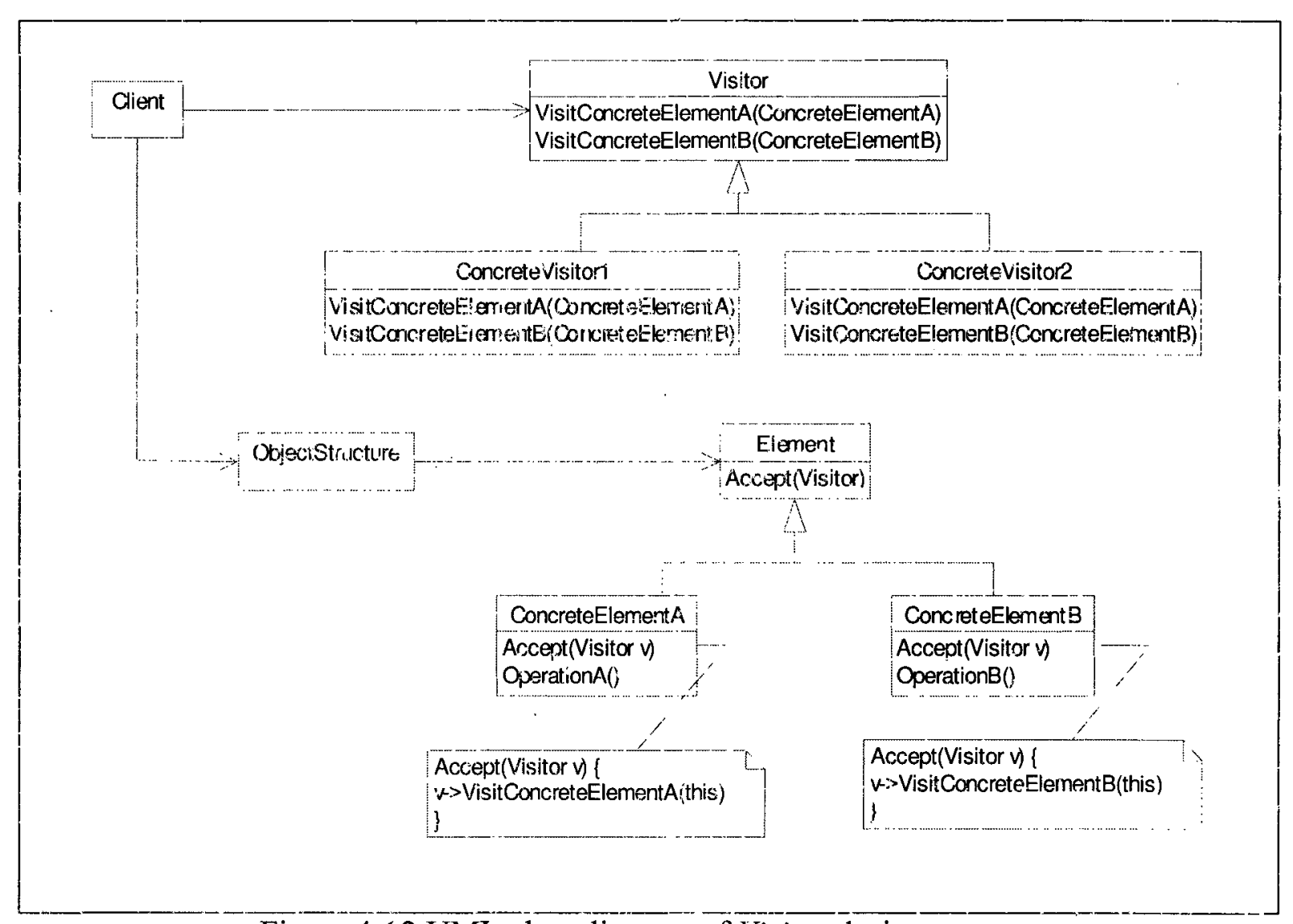

Figure 4-12 UML class diagram of Visitor design patterns

As shown in Figure 4-12 the Visitor class declares a V1sit operation for each class of ConcreteElement and uses them as the argument of the operation. In this way each ConcreteElement class can use Visit operation of Visitor class just like their own if there is an Accept operation with Visitor as an argument in each class of ConcreteElement. 
As defined in section 4.3, Visitor uses each class of ConcreteElement via the arguments of Visit operation. Meanwhile, each class of ConcreteElement delegates Visitor's operations via Accept operation with Visitor as an argument, so there are delegation relationships between Visitor and each class of ConcreteElement. These relaticnships are very useful for identifying this pattern and reflected it in the model below.

\subsection{The mapping between the model of Visitor and its Prolog representation}

The mapping between the model of Visitor and its Prolog representation is as follows:

\begin{tabular}{|l|l|}
\hline Elements in the model & Prolog representation \\
\hline Static Components & vstr_classes \\
Classes & vstr_ops \\
Operations & vstr_sterelations \\
\hline Static Pelationships & inheritance \\
Inheritance & using \\
Using & delegation \\
Delegation & \\
\hline
\end{tabular}

Using Figure 4-12 and the table above, the model of the Visitor design pattern in Prolog: Note: in this table, SC: Static Components; SR: Static Relationships;

DC: Dynamic Components; DR: Dynamic Relationships ; $\quad / * \ldots * /$ : Comments Prolog Representation 


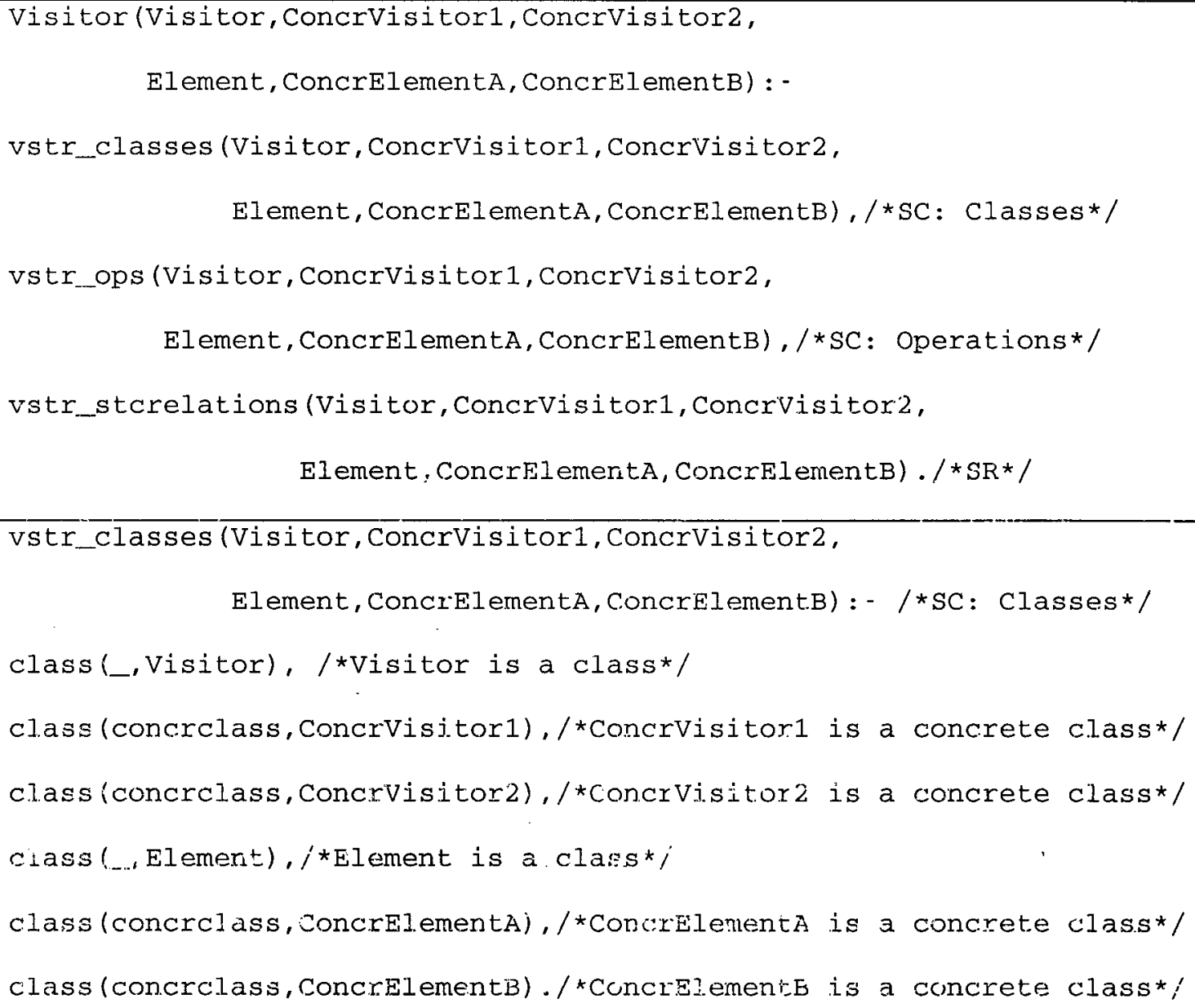




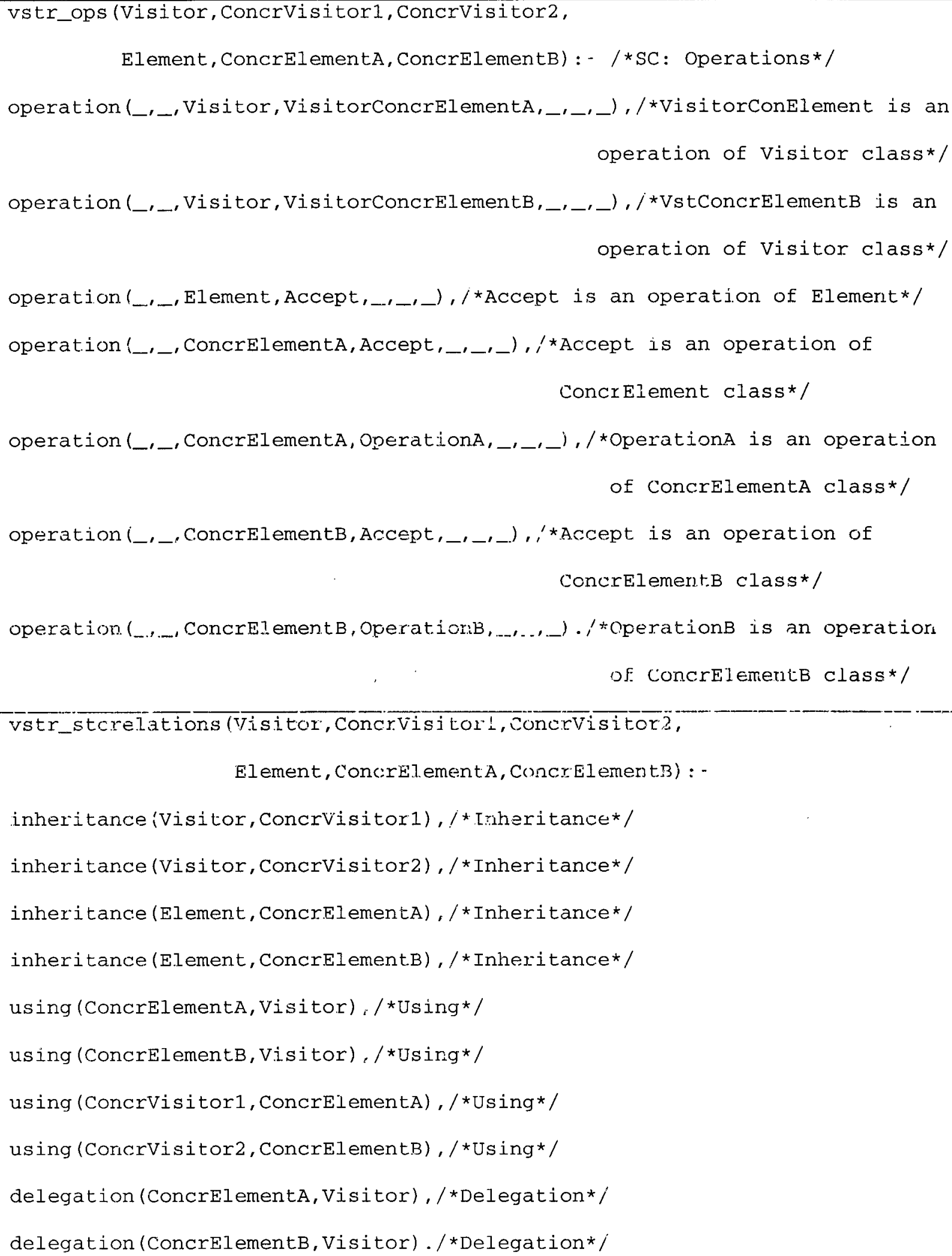




\subsection{Summary}

In this chapter we propose a rule-based approach to the problem of reverse engineering $\mathrm{C}++$ software systems by detecting design patterns. In this approach, design patterns based on static and dynamic information are modeled in logic language. All modeis are composed of components and relationships. Components and relationships are divided into static and dynamic ones respectively. The formal descriptions of some typical patterns in GOF design patterns are presented in details.

In the models for formal descriptions of design patterns, creational design patterns, like Factory Method, are described based on static and dynamic characteristics. Structural design patterns like Adapter are described based on static characteristics because they are of strong structural characteristics. Some behavioral design patterns like strategy are described based on static characteristics and others like Chain of Responsitilitity and Observer are described based on dynamic characteristics because of their strong behaviora! characteristics. 


\section{Chapter 5 Design Pattern Extraction Tool (DPET)}

\subsection{Introduction}

DPET consists of three processes, extraction process, knowledge base process, and pattern detection process.

- Extraction process is made up of a parser, a profiling tool, static and dynamic information repository, and an extractor.

- Knowledge base process is made up of a rule base and a fact base.

- Pattern detection process is made up of a Prolog inference engine.

\subsection{The Architecture of DPET}

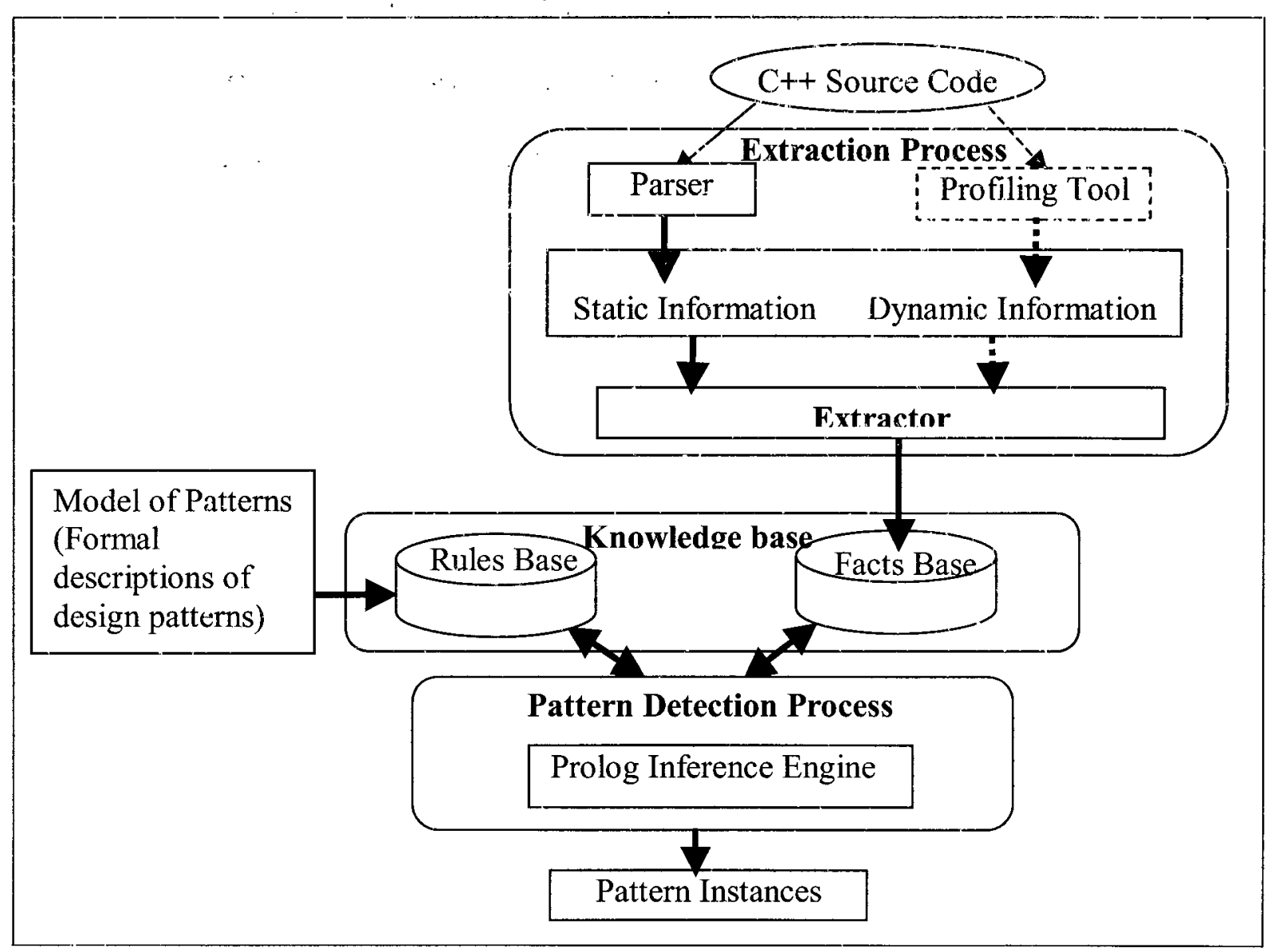

Figure 5-1 The architecture of the tool DPET 
The Design Pattern Extraction Tool (DPET) has been implemented using the approach proposed in Chapter 4. As shown in Figure 5-1, DPET consists of the front-end parser, the profiling tool, the fact and rule base, and the Prolog inference engine.

\subsubsection{The Parser}

A parser for extracting static information from $\mathrm{C}++$ code has been revised based on a $\mathrm{C}++$ parser for measuring software metrics developed by Tim Littlefair[Lit01], which is heavily dependent on ANTLR ( ANother Tool for Language Recognition), a predict $L L(k)$ parser generator [Par93].

ANTLR constructs human-readable recursive-descent parsers in $\mathrm{C}$ or $\mathrm{C}++$ from pred-LL(k) granmars, namely LL(k) grammars, for $\mathrm{k}>1$ that support predicates. Our choice is based on the following properties of ANTLR:

- $\mathrm{k}>1$ lookahead,

- Semantic predicates (the ability to have semantic information direct the parsing)

- Syntactic predicates (selective backtracking).

The pred-LL(k) parsers are stronger than $L R(k)$ parsers for two reasons. First, semantic predicates may be used to parse context sensitive languages. Second, predLL(k) parsers have access to arbitrary lookahead. Further, embedding actions in an LR grammar can introduce ambiguities, thus reducing the strength of LR.

Ultimately, the true test of language tool's usefulness lies with the vast industrial use. ANTLR is widely used in the commercial and academic communities. Thousands of people in virtually all industrialized nations have acquired the software since the original 1.00 release in 1992. Several universities currently teach courses with ANTLR. Many commercial programs use ANTLR [Ant03]. 


\subsubsection{The Profiling Tool}

GNU gprof [Gpr02], a tool for profiling programs, is used to generate dynamic information about source code.

Profiling is a dynamic analysis technique that can provide valuable information about how a program spends its time and which functions called which other functions (Call Graph) while executing. It looks into each function and inserts code at the head and tail of each one in order to collect timing information. Since the profiler uses information collected during the actual execution of the program it can be used on programs that are too large or too complex to analyze by reading the source. That is, profiling technique can provide dynamic information about programs even if we know little about the programs in advance. However, how the program is run will affect the information that shows up in the profile data. If some features of the program are not used while it is being profiled, no profile information will be generated for that feature.

\subsubsection{The Extractor}

The goal of the extractor is to detect relationships among components from program information extracted from source code. The extractor uses the relationship detection algorithms defined in section 4.2.1. The relationships will be used to identify design patterns together with components.

\subsubsection{The Knowledge Base}

The knowledge base consists of two bases, the fact base and the rule base. The design information extracted from source code is stored in the fact base while the model of design patterns is stored in the rule base. 


\subsubsection{Prolog Inference Engine}

By running the Prolog inference engine to query the knowledge base design patterns can be detected.

\subsection{The Extraction Process}

The extraction process of design patterns is introduced briefly in the above section. In order to make it clearer, two pieces of sample code are used to show the whole extraction process. The first code fragment adopted from [Gam95] is used to describe the process of extracting the Proxy design pattern. The second code adopted from our case study-1 is used to describe the process of extracting the Adapter design pattern. Appendix A gives a complete extraction description incliding ihe original data of the intermediate result.

\subsubsection{Extraction Description of Proxy Design Patterns}

\section{- Extraction Process}

(1) Source code is parsed to extract static information using $\mathrm{C}++$ parser

The following sample code fragment is adopted from the "Proxy" sample code in [Gam95]. The Graphic class defines the interface for graphical objects.

The Image class implements the Graphic interface to display image files. Image overrides HandleMouse to let users resize the image interactively.

ImageProxy has the same interface as Image.

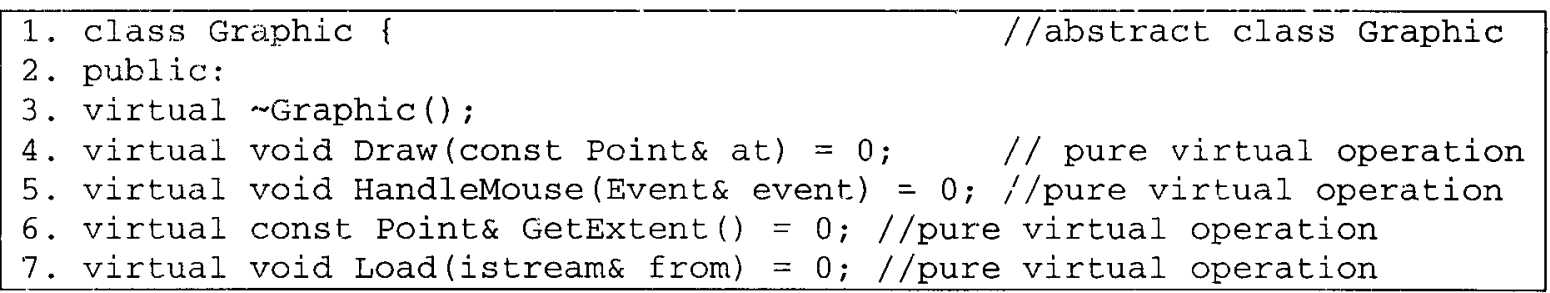




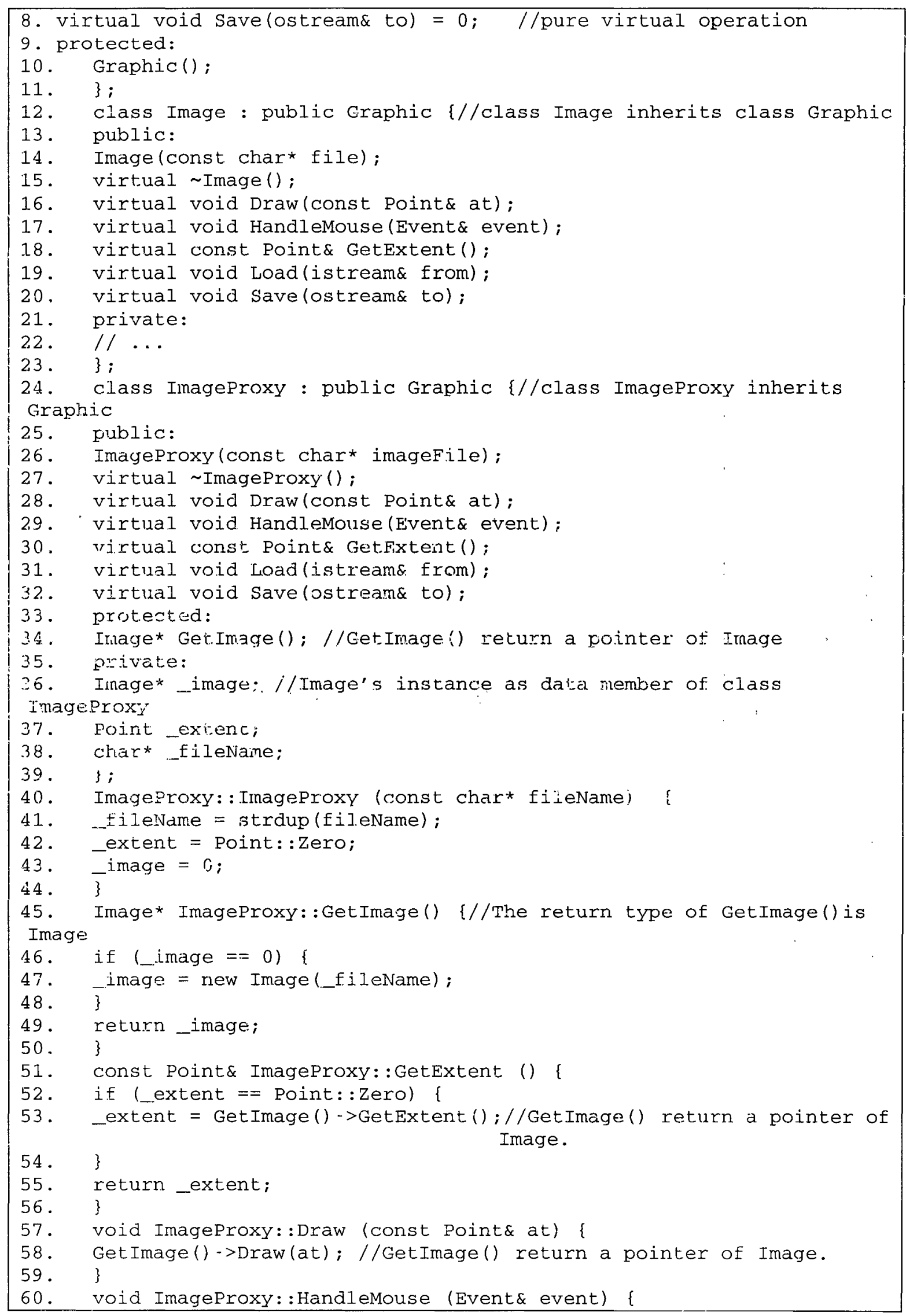




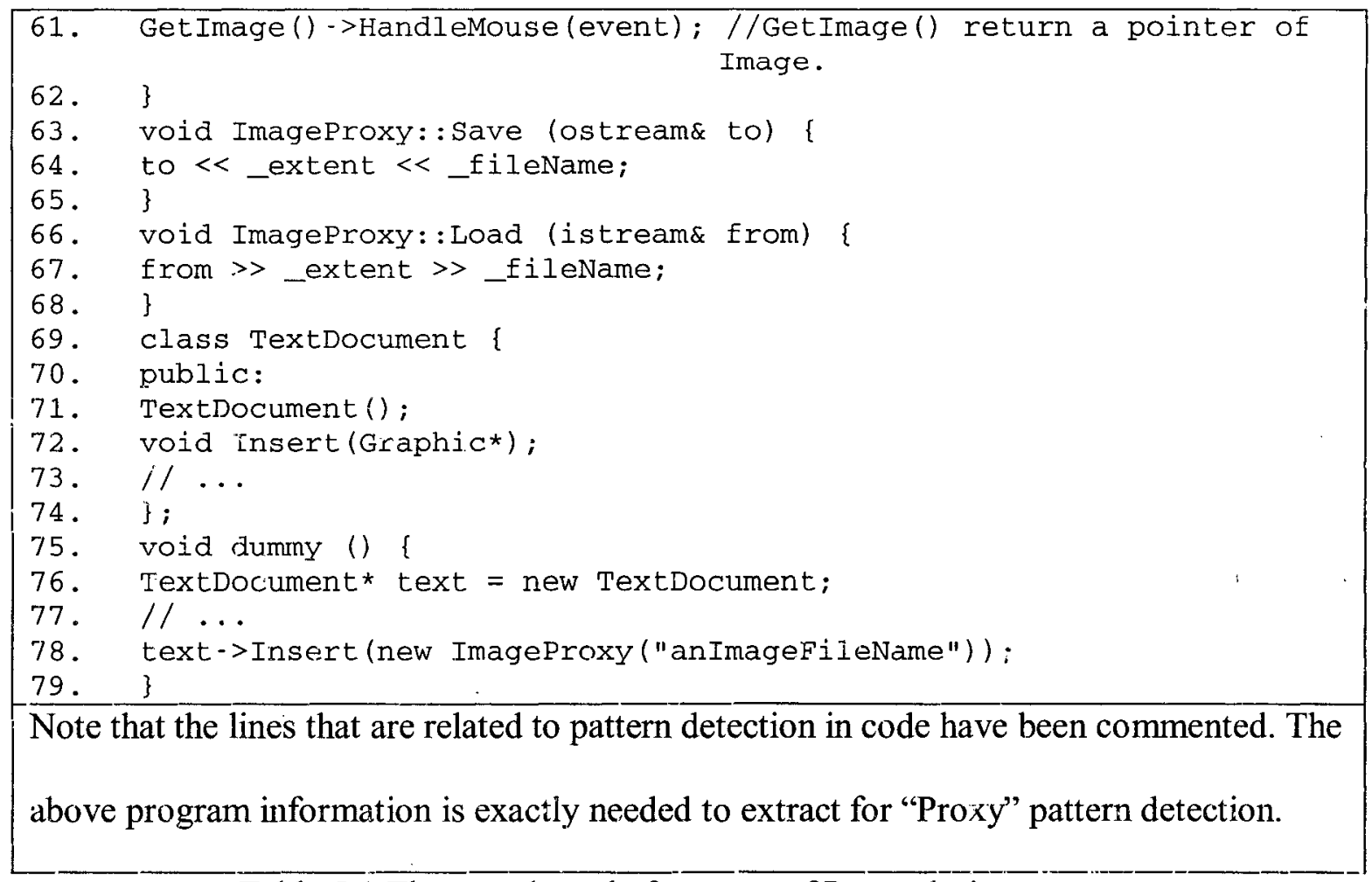

Table 5.1 The sample code fragment of Proxy design patterns

(2) The static information parsed is stored in the below data format:

The data formats used in the extraction of this sample code is shown below. Please refer to Appendix B for a complete data formats and Appendix A for the program information extracted from the above code.

- Data format for Classes:

CCCC_Module@ModuleName@ModuleType

CCCC_ModExt@ModuleName@ModuleType@,

FileName@Line@Definition/Declaration@Properties@Metrics

- ModuleType: Class and Struct

- Meaning: At the Line of the Filename file, there is a Definition/Declaration of ModuleName module of ModuleType (class/struct) type with Properties and Metrics.

- Data format for Operations:

CCCC_Member@ModuleName@OperationName@ReturnType@Parameter@

CCCC_MemExt@ModuleName@OperationName@ReturnType@Parameter@

FileName@Line@Declaration/Definition@OperationType@Metrics

- Meaning: At the Line of the FileName file, there is a Definition/Declaration of OperationName of OperationType in ModuleName with ReturnType, Parameter 
and Metrics.

- Data format for basic relationships among classes (pass by reference, has by value, inheritance) and class attribute: data member in class.

CCCC_UseRel@ModuleName1@ModuleName2@(Pointer/Reference)@

CCCC_UseExt@ModuleName1@ModuleName2@(Pointer/Reference)

@FileName@Line@BasicRelationships@Properties@Metrics

- Meaning: At the Line of the FileName file, there is a basic Relationship between ModuleName1 and ModuleName2 with Properties and Metrics.

- Data Format for Delegation Instances: CCCC_Delegation@Reference/Pointer@CalledOperation@CallingModule@ CallingOperation@

- Meaning: The CallingOperation of the CallingModule calls the CalledOperation with the Refence/Pointer.

(3) The design information (e.g., classes and their interrelationships) is extracted from the static information using the Extractor, and stored in the fact base of the knowledge base.

\section{- Knowledge base process}

(1) The facts stored in the fact base as follows:

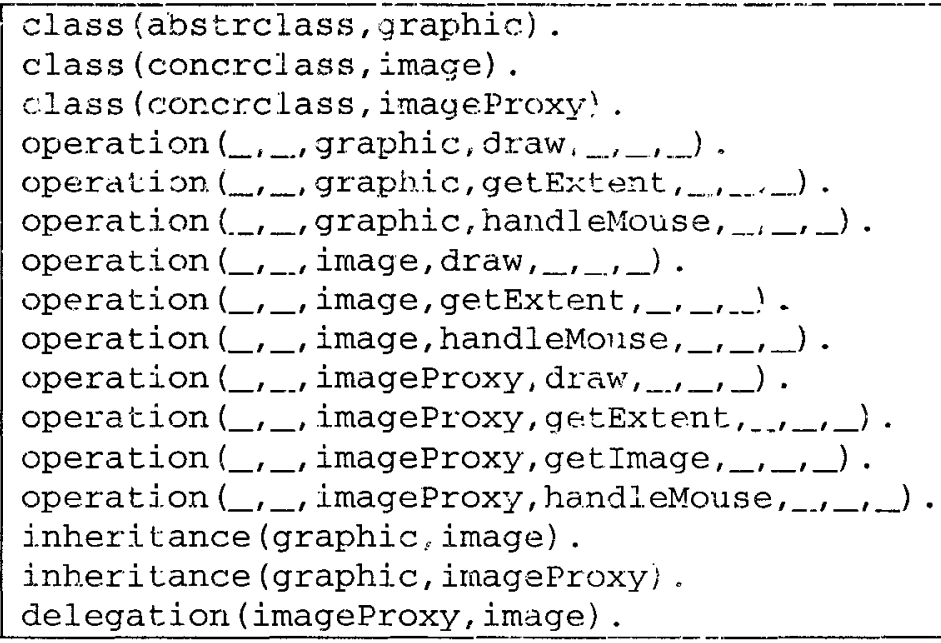

(2) The rules for identifying the "Proxy" design pattern is stored in the rule base of the

knowledge base based on the model of formal descriptions of design patterns.

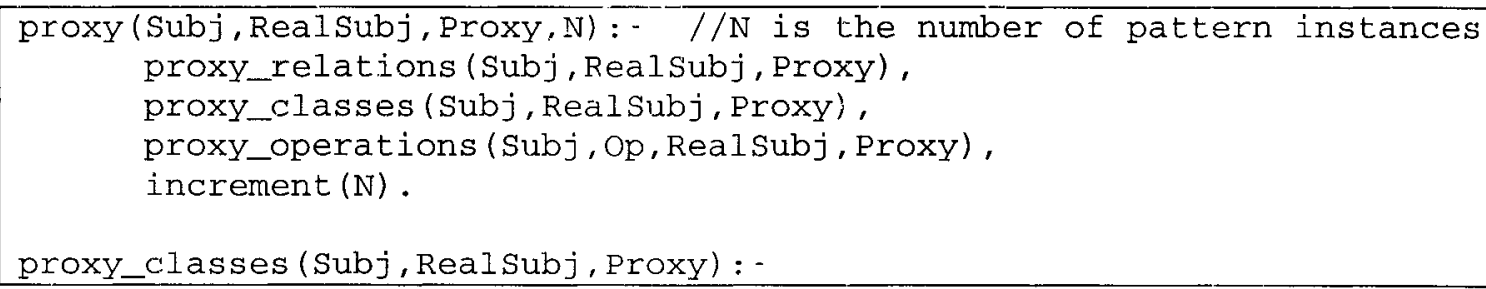




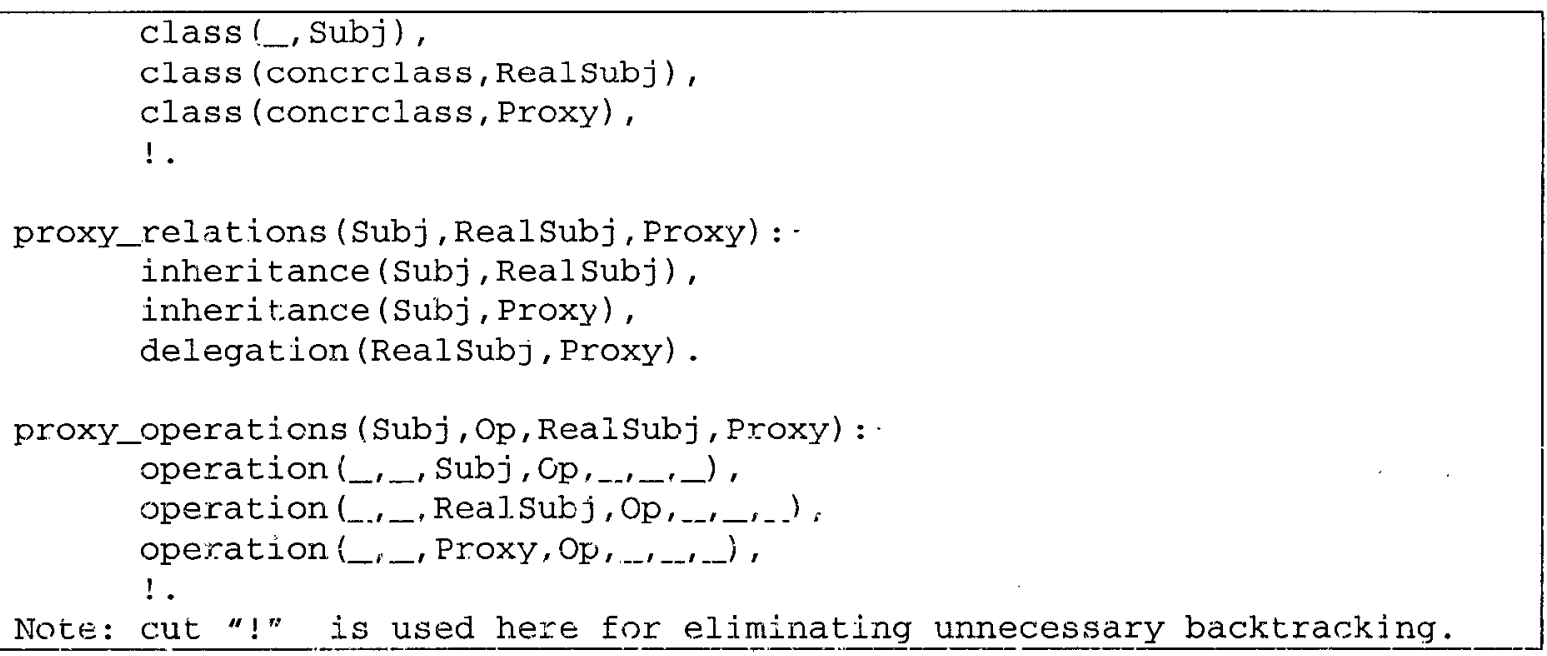

\section{- Pattern Detection Process}

The design pattern can be detected by querying the knowledge base.

The following results are obtained after running SWI-Prolog inference engine to identify design patterns:

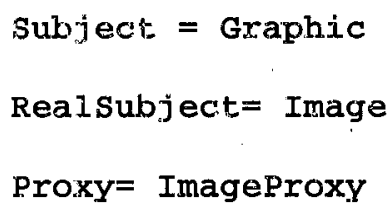

It indicates that there is a "Proxy" design pattern in the code that consists of Graphic class, Inage class and ImageProxy class as shown in Figure 5-2. 


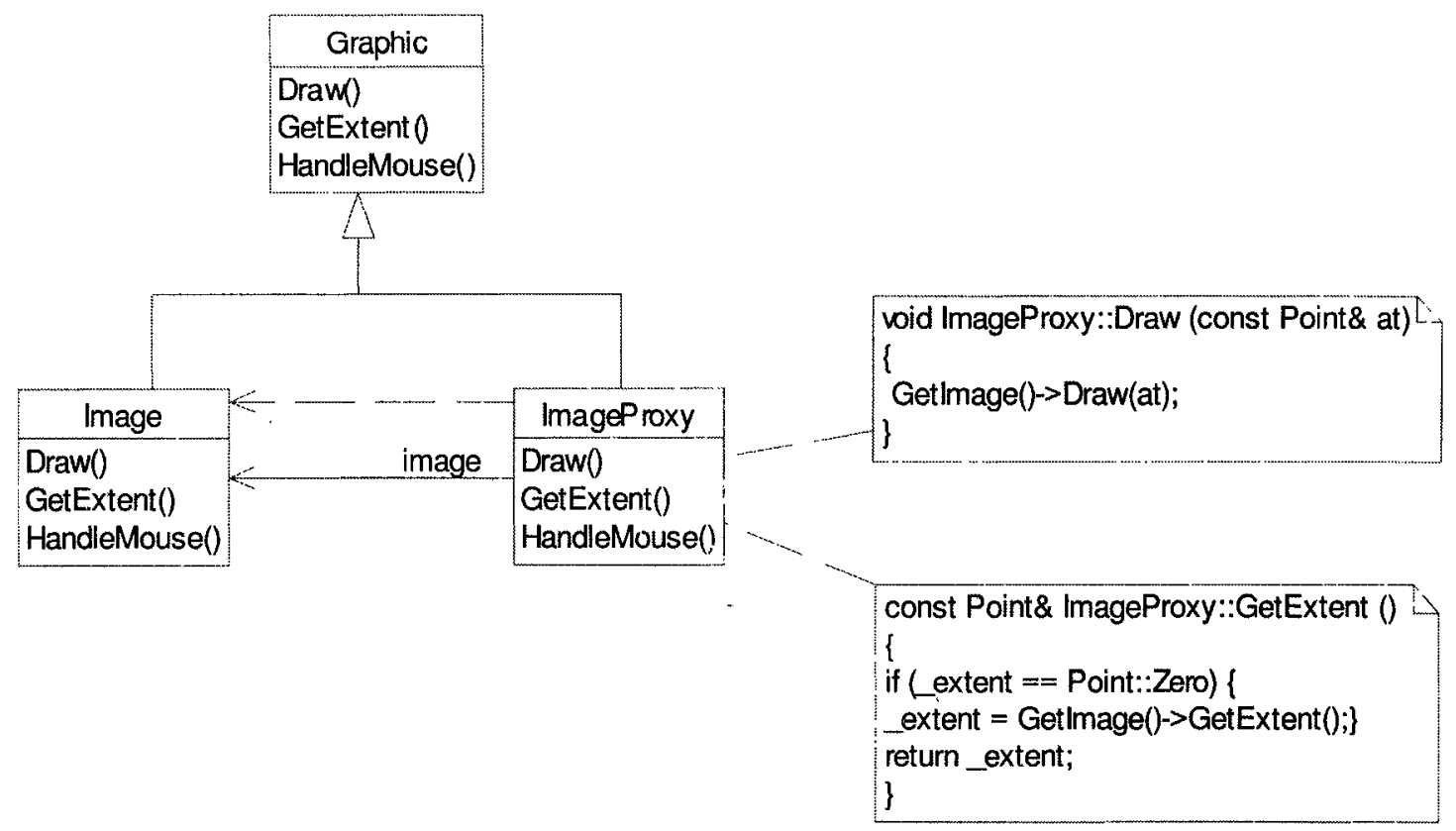

Figure 5-2 UML representation of the "Proxy" design pattern

\subsubsection{Extraction Description of Adapter Design Patterns}

\section{- Extraction Process}

(1) Scurce code is parsed to extract static information using $\mathrm{C}++$ parser.

The following sample code fragment is from LQNS that is applied in case study-1 later. ActivityList class is an interface; SeqentialActivityList class inherits from it and overrides owner operation by delegating owner operation of Activity class.

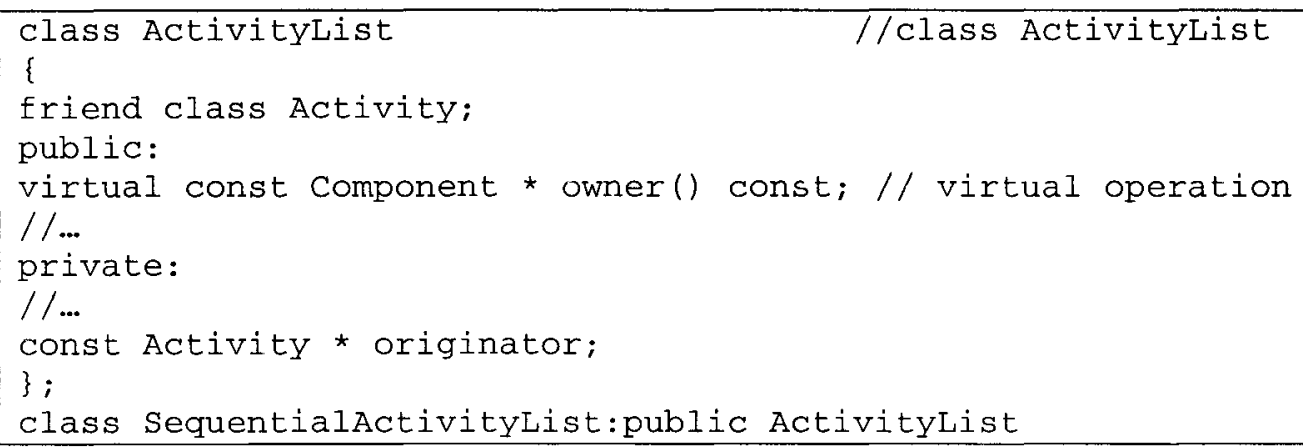




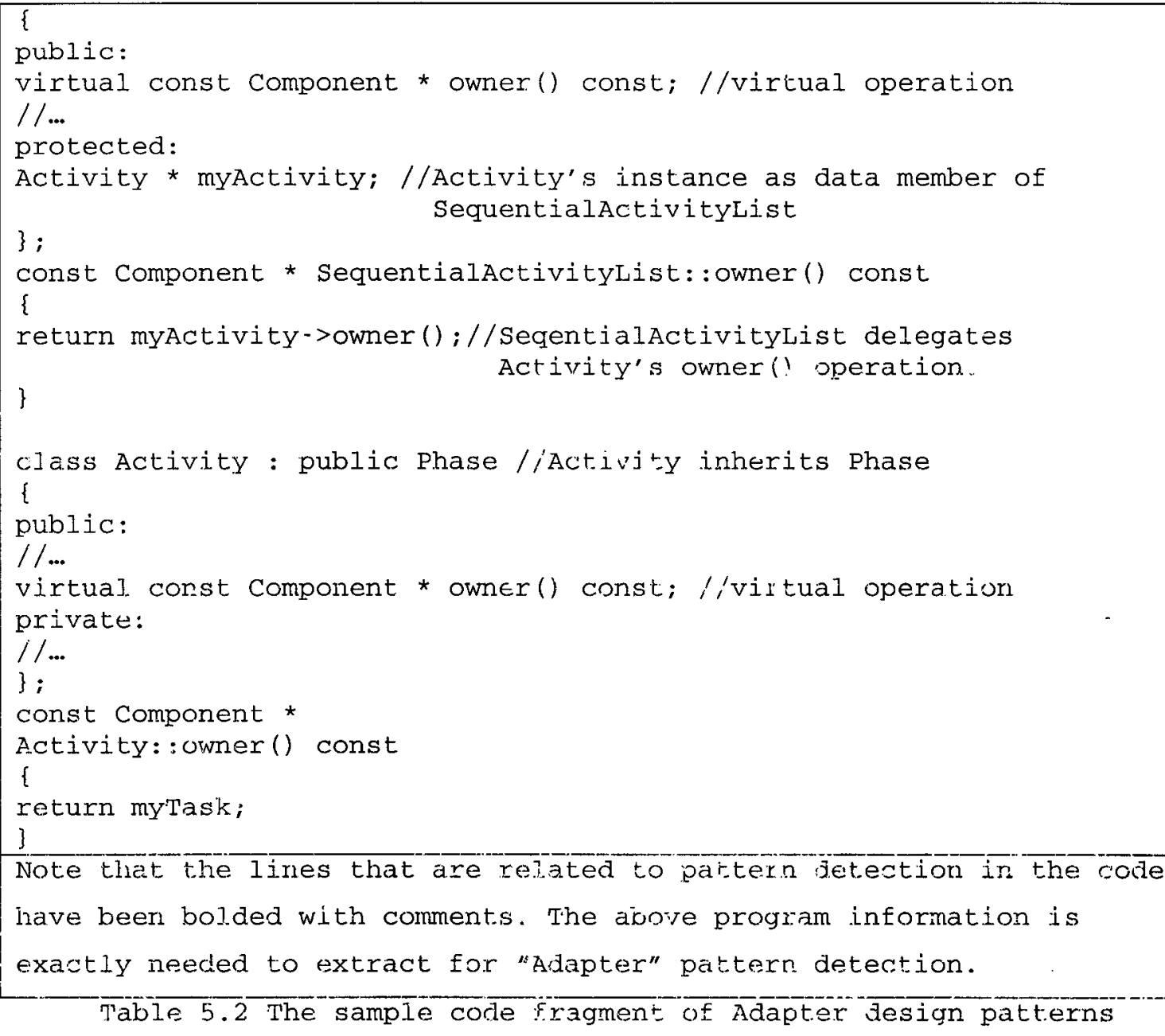

(2) The static information parsed is stored in the below data formats:

The data formats used in the extraction of this sample code is shown below. Please refer to Appendix B for a complete data formats and Appendix A for the program information extracted from the above code.

- Data format for Classes:

CCCC_Module@ModuleName@ModuleType

CCCC_ModExt@ModuleName@ModuleType(a)

FileName@Line@Definition/Declaration@Properties@Metrics

- ModuleType: Class and Struct

- Meaning: At the Line of the Filename file, there is a definition/declaration of ModuleName module of ModuleType (class/struct) type with Properties and Metrics.

- Data format for Operations: 
CCCC_Member@ModuleName@OperationName@ReturnType@Parameter@

CCCC_MemExt@ModuleName@OperationName@ReturnType@Parameter@

FileName@Line@Declaration/Definition@OperationType@Metrics

- Meaning: At the Line of the FileName file, there is an Definition/Declaration of OperationName of OperationType in ModuleName with ReturnType, Parameter and Metrics.

- Data format for basic relationships among classes (pass by reference, has by value, inheritance) and class attribute: data member in class.

CCCC_UseRel@ModuleName1@ModuleName2@(Pointer/Reference)@,

CCCC_UseExt@ModuleName1@ModuleName2@(Pointer/Reference)

@FileName@Line@BasicRelationships@Properties@Metrics

- Meaning: At the Line of the FileName file, there is a basic Relationship between ModuleNamel and ModuleName2 with Properties and Metrics

- Data Format for Delegation Instances:

CCCC_Delegation@Reference/Pointer@CalledOperation@CallingModule@,

CallingOperation@

- Meaning: The CallingOperation of the CallingModule calls the CalledOperation with the Refence/Pointer.

(3) The design information (e.g., classes and their interrelationships) is extracted from the static information using the Extractor, and stored in the fact base of the krowledge base.

\section{- Knowledge base process}

(1) The facts stored in the fact base as follows:

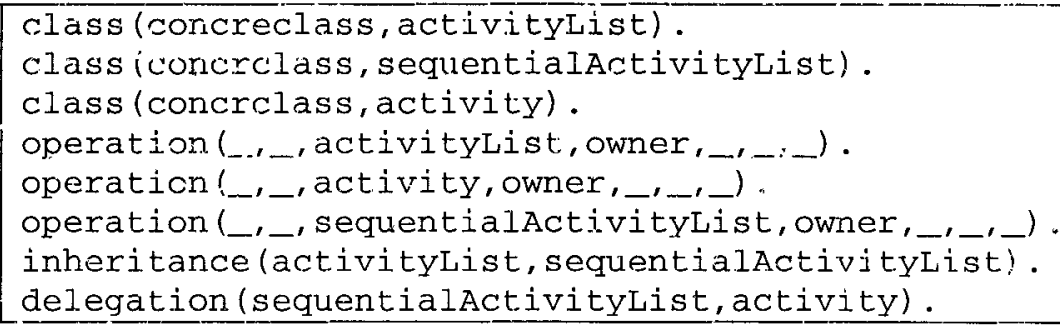

(2) The rules for identifying the "Proxy" design pattern is stored in the rule base of the

knowledge base based on the model of formal description of design patterns.

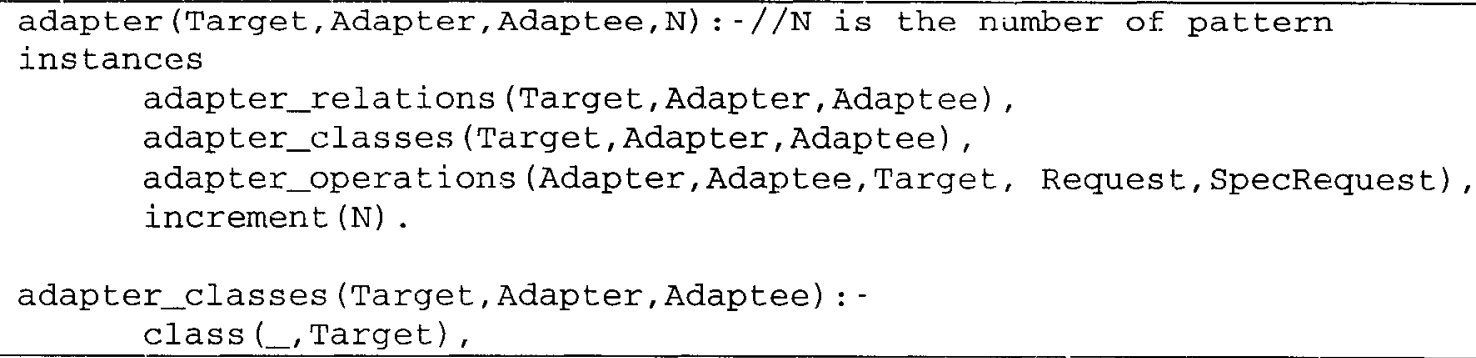




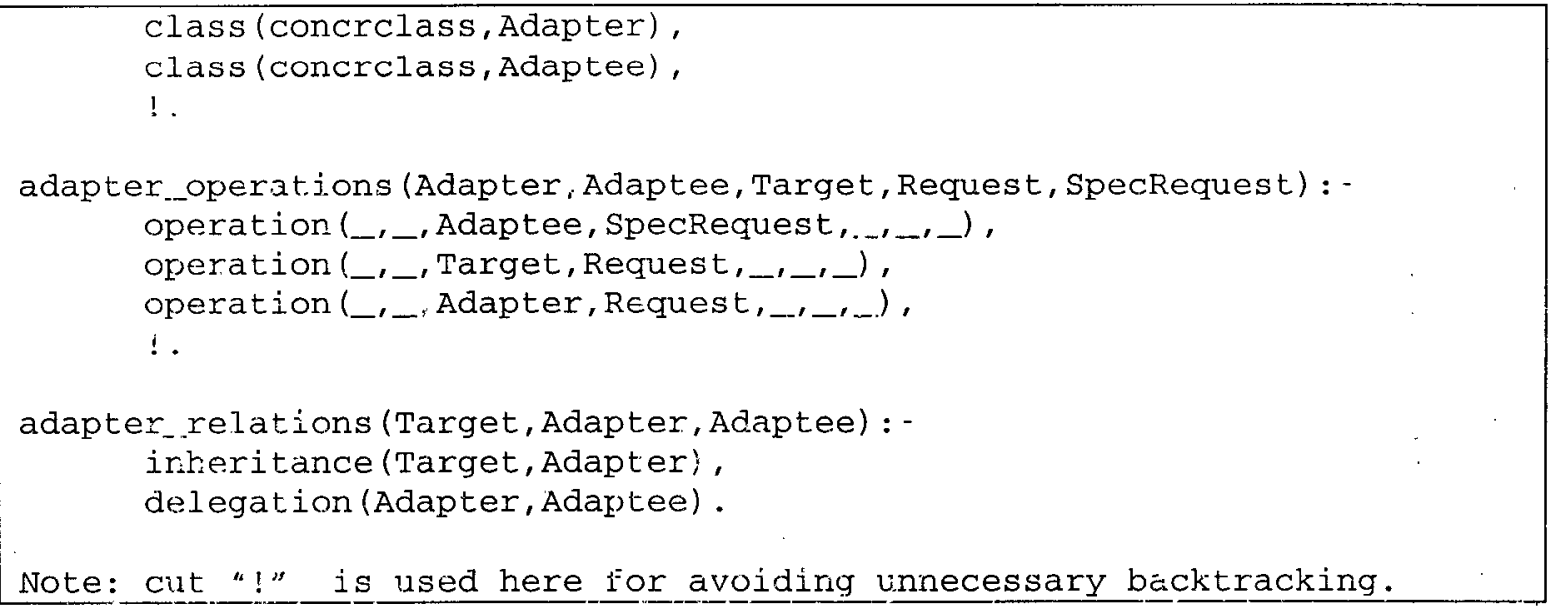

\section{- Pattern Detection Process}

The design pattern can be detected by querying the knowledge base.

The results after running SWI-Prolog inference engine to identify design patterns:

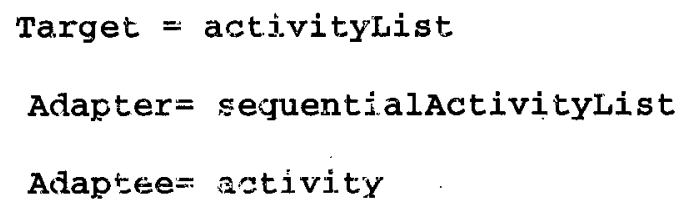

It indicates that there is an "Adapter" design pattern in the code, which consists of activitylist ciass, sequentialActivityList class and activity class as shown in Figure 5-3.

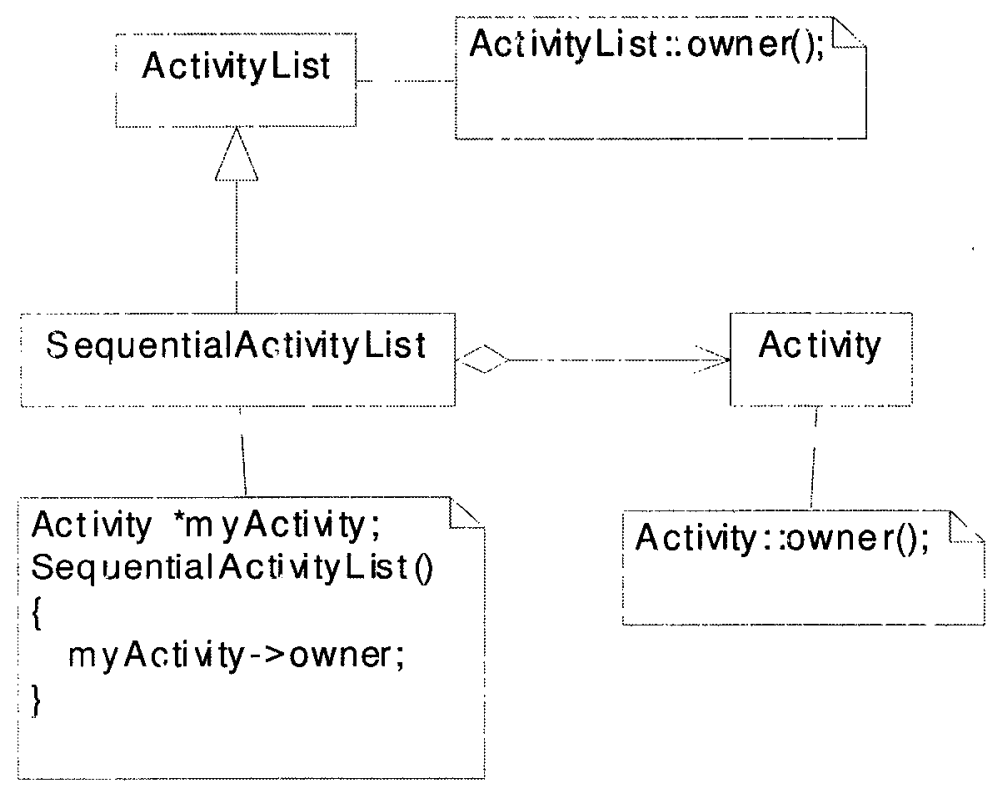


Figure 5-3 UML representation of the "Adapter" design pattern

\subsubsection{Extraction Description of Dynamic Information using Profiling}

GNU gprof, a tool for profiling programs is used to capture dynamic information. The sample code adopted from [Gam95] for the Chain of Responsibility design pattern has been profiled by GNU gprof. The call graphs about the sample code have been obtained.

In order to profile a program the following steps are taken:

(1) Compile and link the program with profiling enabled.

To compile and link a source file for profiling, specify the -pg'option when running the compiler.

Here are examples: g++ -pg chain.c -o chain (chain.C is shown in Table 5.3)

(2) Execute the program to generate a profile data file. chain

Once the program is compiled for profiling run it in order to generate the information that gprof needs. Simply run the program as usual using the normal arguments, file names, etc. The program should run normally producing the same output as usual. It will, however, run somewhat slower than normal because of the time spent collecting and the writing the profile data.

The program will write the profile data into a file called 'gmon.out' just before exiting.

(3) Run gprof to analyze the profile data. 
gprof - $\mathrm{c}-\mathrm{z}$ chain gmon.out

After you have a profile data file 'gmon.out', run gprof to interpret the information

in it. The gprof program prints a flat profile and a call graph on standard output.

Typically redirect the output of gprof into a file with ' $>$ '.

Run gprof like this:

gprof options [executable-file [profile-data-files...]] [> outfile]

Here square brackets indicate optional arguments.

The following sample code is adopted from [Gam95], which originally is a piece of code fragment and cannot be executed; to execute it some modifications are made. 'This example illustrates how a chain of responsibility can handle requests for an online help system. The user can obtain help information on any part of the graphical interface just by clicking on it. The help that's provided depends on the part of the interface that's selected and its context. The button in a dialog box offers information, if specific help information exists for that part of the interface. If not a more general help message-dialog box should be displayed. If there is no help information from a dialog box a more general dialog box should appear-application offers help information to its users. In this way the help request can be passed along the chain formed by button, dialog, and application objects.

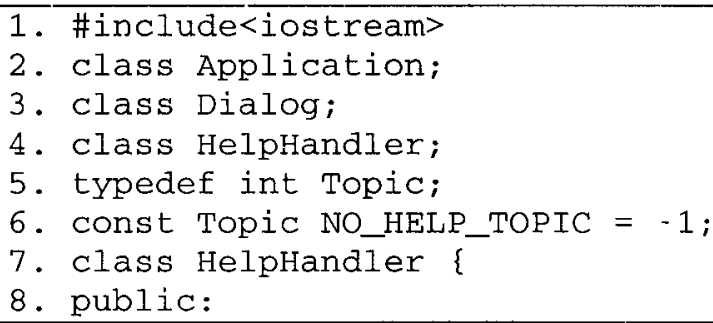




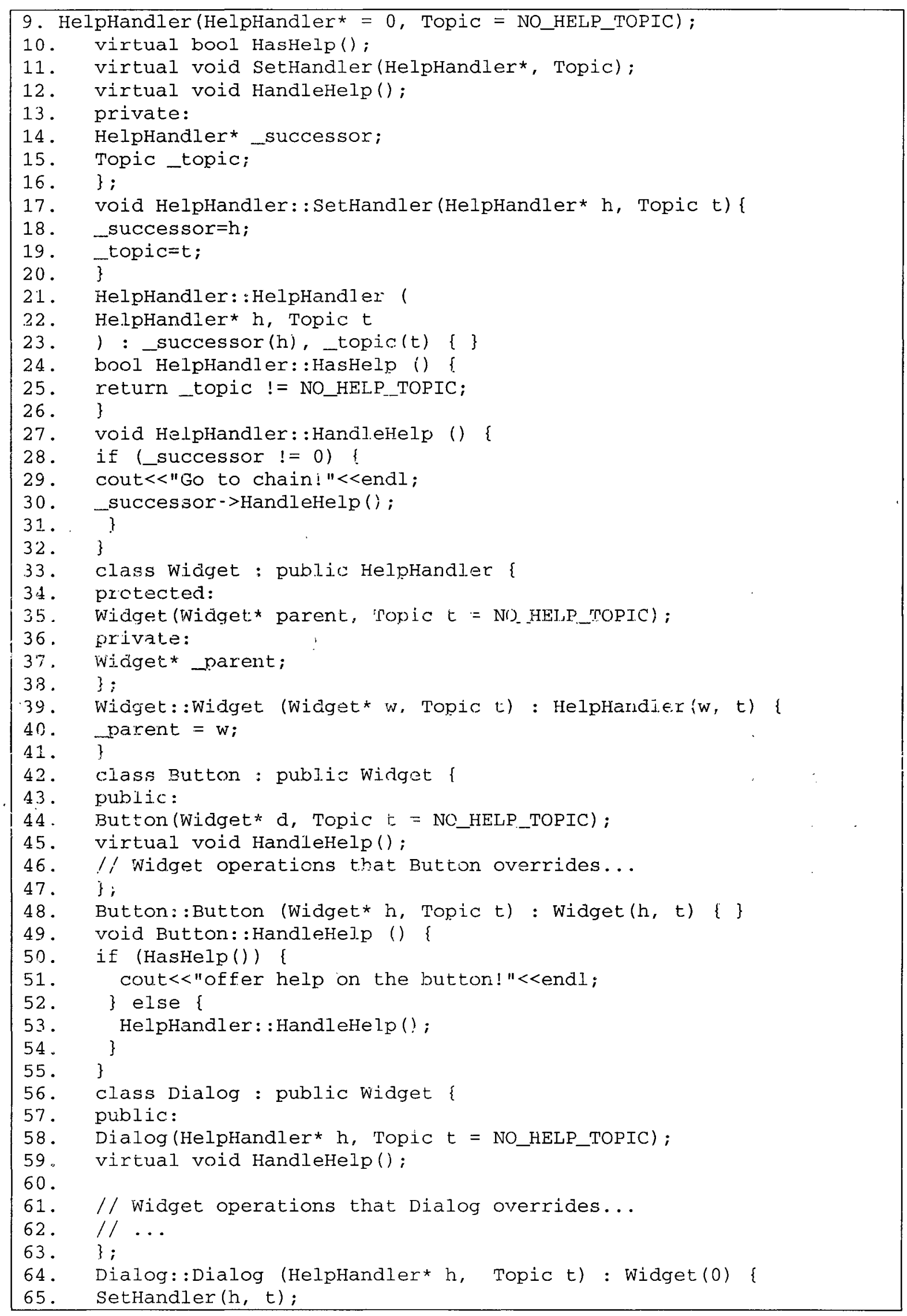




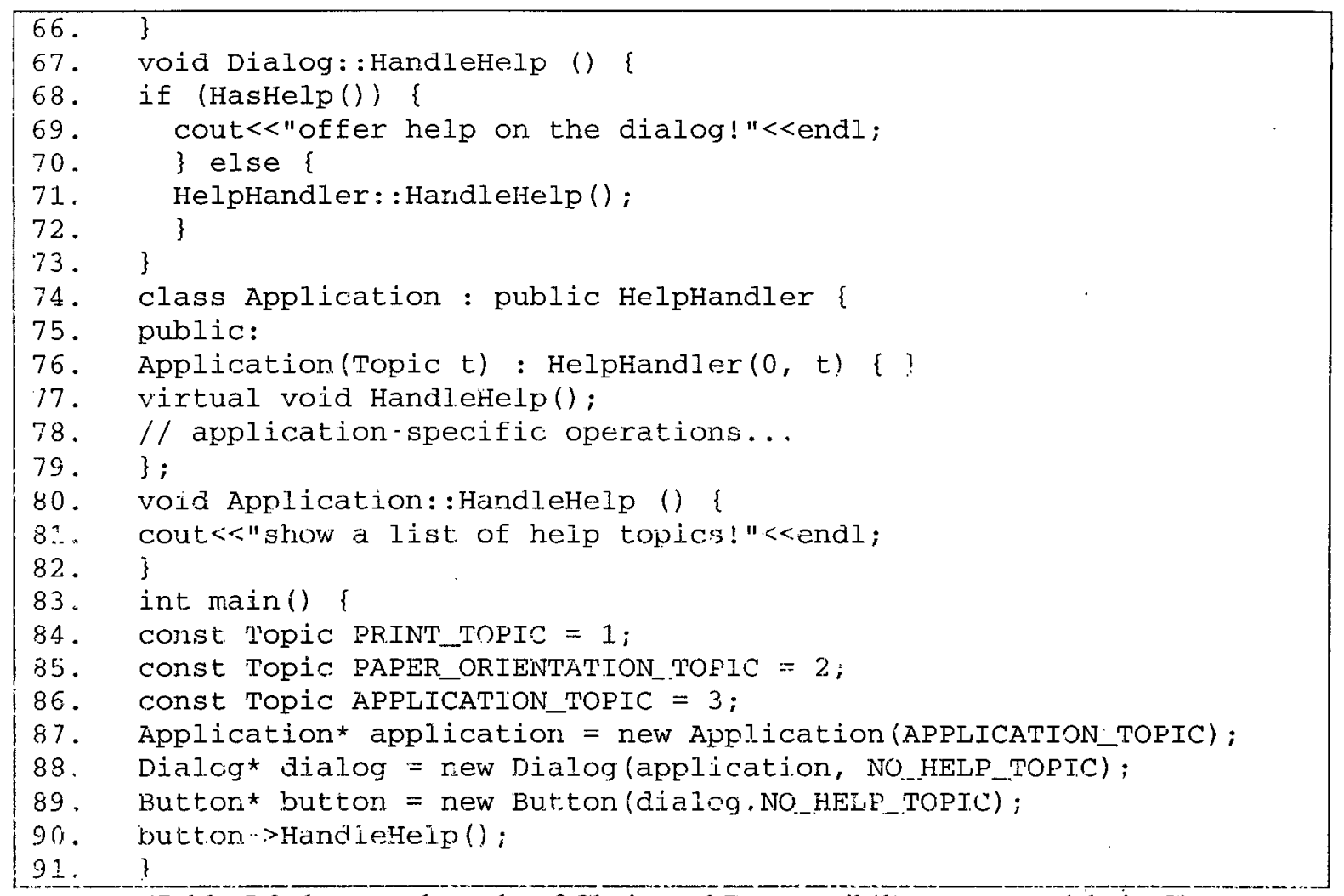

Table 5.3 the sample code of Chain atd Responsibility patterns (shain. $\bar{C}$ )

The Output is obtained atter running grofof cormmand. The related part of the call graph is shown below for giving an overview. Please see Appendix $\mathrm{C}$ for details.

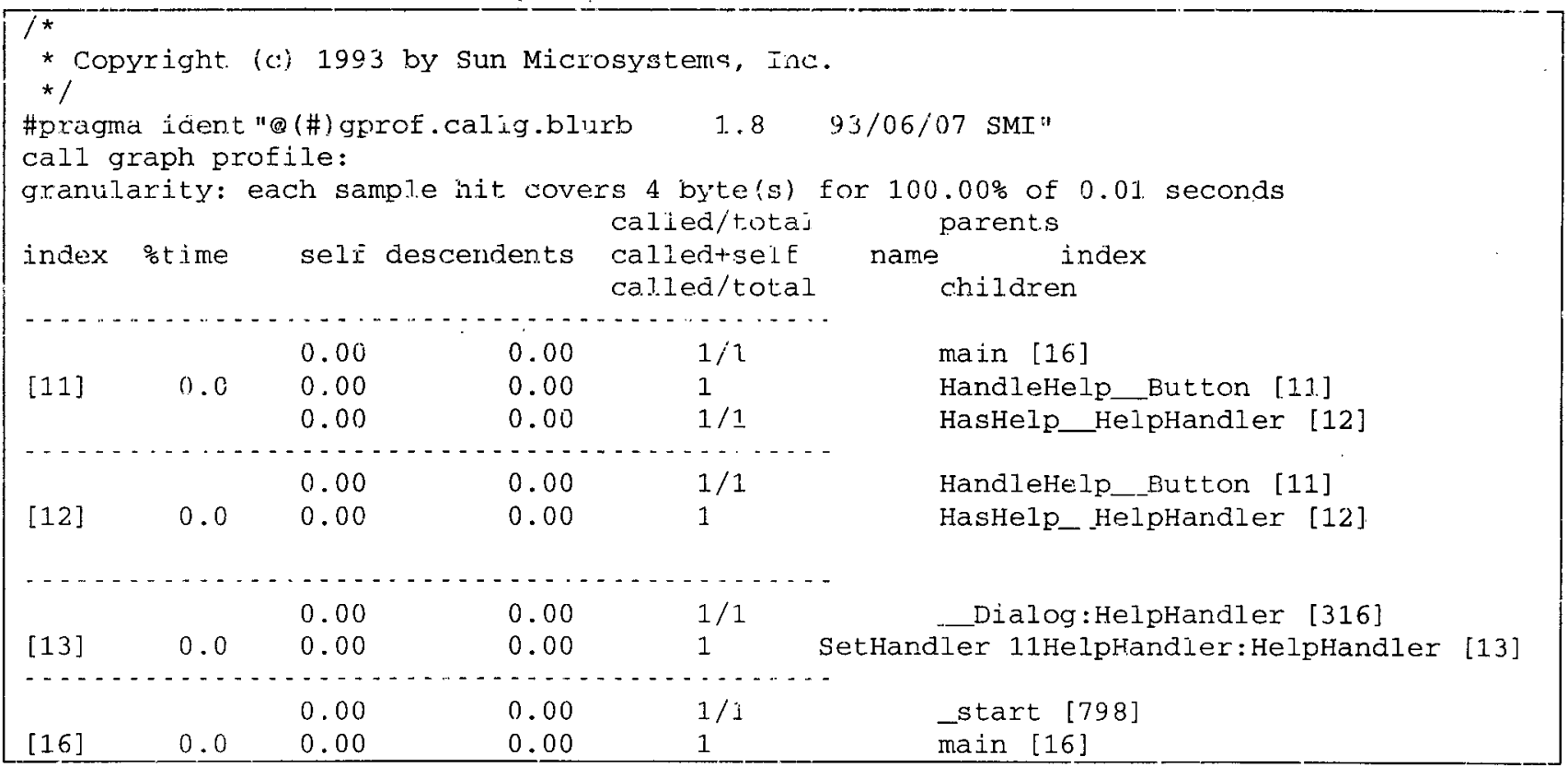




\begin{tabular}{|c|c|c|c|c|c|}
\hline & & $\begin{array}{l}0.00 \\
0.00 \\
0.00 \\
0.00\end{array}$ & $\begin{array}{l}0.00 \\
0.00 \\
0.00 \\
0.00\end{array}$ & $\begin{array}{l}1 / 1 \\
1 / 1 \\
1 / 1 \\
1 / 1\end{array}$ & $\begin{array}{l}\text { Application [314] } \\
\text { Dialog:HelpHandler [316] } \\
\text { Button:Widget [315] } \\
\text { HandleHelp_Button [11] }\end{array}$ \\
\hline$[306]$ & 0.0 & $\begin{array}{l}0.00 \\
0.00 \\
0.00\end{array}$ & $\begin{array}{l}0.00 \\
0.00 \\
0.00\end{array}$ & $\begin{array}{l}1 / 3 \\
2 / 3 \\
3\end{array}$ & $\begin{array}{l}\text { Application [314] } \\
\text { Widget:Widget [308] } \\
\text { HelpHandler: HelpHandler [306] }\end{array}$ \\
\hline$\cdots$ & $\cdots$ & $\begin{array}{l}0.00 \\
0.00 \\
0.00 \\
0.00\end{array}$ & $\begin{array}{l}0.00 \\
0.00 \\
0.00 \\
0.00\end{array}$ & $\begin{array}{l}1 / 2 \\
1 / 2 \\
2 \\
2 / 3\end{array}$ & $\begin{array}{l}\text { Button:Widget [315] } \\
\text {-Dialog: HelpHandler [316] } \\
\text { - Widget:Widget [308] } \\
\text { HelpHandler:HelpHandler }\end{array}$ \\
\hline [314] & 0.0 & $\begin{array}{l}0.00 \\
0.00 \\
0.00\end{array}$ & $\begin{array}{l}0.00 \\
0.00 \\
0.00\end{array}$ & $\begin{array}{l}1 / 1 \\
1 \\
1 / 3\end{array}$ & $\begin{array}{l}\text { main [16] } \\
\text { _Appl ication [314] } \\
\text { _HeipHandler: HelpHandler [306] }\end{array}$ \\
\hline$[315]$ & 0.0 & $\begin{array}{l}0.00 \\
0.00 \\
0.00\end{array}$ & $\begin{array}{l}0.00 \\
0.00 \\
0.00\end{array}$ & $\begin{array}{l}1 / 1 \\
1 \\
1 / 2\end{array}$ & $\begin{array}{l}\text { main [16] } \\
\text {-_Button:Widget [315] } \\
\text { Widget:widget [308] }\end{array}$ \\
\hline [316] & 0.0 & $\begin{array}{l}0.00 \\
0.00 \\
0.00 \\
0.00\end{array}$ & $\begin{array}{l}0.00 \\
0.00 \\
0.00 \\
0.00\end{array}$ & $\begin{array}{l}1 / 1 \\
1 \\
1 / 2 \\
1 / 1\end{array}$ & $\begin{array}{l}\text { main [16] } \\
\text { Dialog: HelpHandler [316] } \\
\text { Widget:Widget [308] } \\
\text { dler.HelpHand] er:HelpHandler [13] }\end{array}$ \\
\hline $\begin{array}{r}\text { Index } \\
{[8]} \\
{[11]} \\
{[12]} \\
{[13]} \\
{[314]} \\
{[306]} \\
{[315]} \\
{[316]} \\
{[308]} \\
\text { Objec } \\
1 .: \text { ch }\end{array}$ & 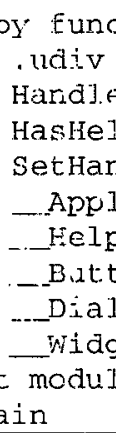 & $\begin{array}{l}\text { on name } \\
\text { lp_Button } \\
\text { HelpHand } \\
\text { er HelpH } \\
\text { ation } \\
\text { ndler:H } \\
\text { : Widget } \\
\text { : HelpHan } \\
\text { : Widget }\end{array}$ & $\begin{array}{r}{[323]} \\
{[324]} \\
{[325]} \\
{[326]} \\
{[327]} \\
{[328]} \\
{[321]} \\
{[3]} \\
{[4]}\end{array}$ & $\begin{array}{l}\text { _getegid } \\
\text { _geteuid } \\
\text { _getgia } \\
\text { _getuid } \\
\text { _ioct } 1 \\
\text { _isatty } \\
\text { _lseek } 4 \\
\text { _malloc_uniocked } \\
\text { _morecore }\end{array}$ & $\begin{array}{l}{[332] \text { _setorientation }} \\
{[307] \text { - sma]loc }} \\
{[333] \text { - thr_main }} \\
{[334] \text {-write }} \\
{[335] \text {.wrt_chi }} \\
{[336] \text {-xf].sbur }} \\
{[6] \text { atexit }} \\
{[14] \text { check_nlspath_env }} \\
{[5] \text { cieanfree }}\end{array}$ \\
\hline
\end{tabular}

From the above call graph we can see the parents and children of each operation used during run time, that is, which operation calls for which other operation. But in the call graph there is no information about the calling order among operations. The sequence of objects sending messages cannot be induced, this is important in identifying some behavioral design patterns. 


\subsection{Summary}

In DPET, the extraction process of design information is language- specific. This is because different object-oriented languages have different implementations for the relationships. For example, in $\mathrm{C}++$ there is a pointer of a class as a data member of another class to form an aggregation relationship between two classes. But in Java there is no pointer at all. So the relationships detection algorithm has to be updated according to different implementations among $\mathrm{OO}$ languages.

Meanwhile, the pattern detection process is language-independent because logic language Prolog has been adopted to represent the model of formal descriptions of design patterns. Prolog representation is language-independent and the design pattern extraction may be applied to other language such as Java or Smalltalk, etc. without changing the rules of design patterns.

In addition to this new rules may be added into the model in the future. Logic language makes this easy to achieve this. 


\section{Chapter 6 Case Study}

\subsection{Introduction}

In order to validate our approach we have applied DPET to the two case studies.

The first case study is the extraction of design patterns from LQNS (Layered

Queuing Network Solver), a C++ source code. LQNS is a tool for the evaluation of software performance that was developed by Woodside et al [Fra99, Fra96] at the department of Systems and Computer Engineering, Carleton University. LQNS provides a language for modeling layered systems. LQNS has been selected for this research project for two reasons. Firstly, we can easily access its complete source code and its design documentation. This enables us to compare the pattern instances extracted by the DPET with design documentation to make sure whether the results are correct. Secondly, LQNS is a large-sized software system that implies that our approach can be applied to large--sized software systems in public domain.

The second case study is based on the extraction of design patterns from three pieces of $\mathrm{C}++$ source code: libg++, galib, and groff. Libg++(version 2.7.2) is a wellknown $\mathrm{C}++$ library of foundation class and is part of the GNU Free Software Foundation $\mathrm{C}++$ development environment. Galib (release 2.4), developed at the Massachusetts Institute of Technology, is a C++ Genetic Algorithm Library to solve optimizations problems. Groff (version 1.10) is the GNU Free Software Foundation version of Unix groff utility. Because these three pieces of software were used in [Ant98] to validate the that particular proposed approach we selected them for our case studies in order to 
compare the results of our approach with those obtained by the Multi-stage filtering approach (MsA) from [Ant98].

\subsection{Case Study-1}

In this case study the tool DPET has been used to extract design patterns from the source code of LQNS. There are total nine kinds of patterns to be detected that are selected from creational, structural and behavioral design patterns respectively: The design information obtained by this case study is compared with the design documentation to make a conclusion.

The following table shows that LQNS has 279 classes and complex relationships between classes.

\begin{tabular}{|l|l|l|l|}
\hline LOC & 21,132 & Associations & 16 \\
\hline Classes & 279 & Using & 700 \\
\hline Operations & $1,91 !$ & Delegations & 28 \\
\hline Aggregations & 142 & Detection Time $(\mathrm{sec})$ & 131 \\
\hline
\end{tabular}

Table 6.1 LQNS code characteristics and detection time

\subsubsection{The Results of Case Study-1}

We tried to extract GOF design pattern instances from LQNS source code. Table 6.2 shows the results of extraction. The number of real patterns in LQNS code has been obtained by checking both the design documentation and the source code of LQNS. 


\begin{tabular}{|c|c|c|c|c|}
\hline Code & \multicolumn{4}{|l|}{ LQNS } \\
\hline Pattern & Pattern instances & Real patterns & False Positives & Precision \\
\hline Factory Method & 0 & 0 & 0 & - \\
\hline Adapter & 12 & 8 & 4 & $67 \%$ \\
\hline Proxy & 1 & $\overline{1}$ & 0 & $100 \%$ \\
\hline Bridge & 0 & 0 & 0 & - \\
\hline Composite & 0 & 0 & 0 & - \\
\hline Decorator & 0 & 0 & 0 & - \\
\hline Strategy & 0 & 0 & 0 & - \\
\hline Visitor & 0 & 0 & 0 & - \\
\hline Observer & 7 & - & 7 & - \\
\hline Time (sec) & \multicolumn{4}{|l|}{131} \\
\hline
\end{tabular}

Table 6.2 Results of pattern detection in LQNS using our tool $D P E T$

\subsubsection{Discussion on Case Study-1}

The extraction process took 131 seconds and it produced (Table 6.1) 279 classes, 1,911 operations, 142 aggregations, 16 associations, 193 inheritances, and 700 using relationships When comparing the results with the design docurnentation and the source code there were twelve instances of adapter patterns compared to eight real patterns giving four false positive patterns. The precision is sixty seven percent. The second pattern extracted was the proxy pattern. In this case there is $100 \%$ precision. We intentionally tried to only use structure-based model (Static Component \& Static Relationships) to identify behavioral design patterns Observer. It resulted in 7 false positives of Observer pattern instances. The reason why we got them is that some behavioral design patterns like Observer and Mediator have strong dynamic characteristics as well as weak static characteristics. This is because they are responsible for the behavior of decoupling senders and receivers. Without dynamic information we 
may get many false positives. It would be effective to reduce the false positives and augment the precision of retrieval if true behavioral characteristics are identified.

\subsection{Case Study-2}

In order to show what we have achieved in comparison with the approach proposed in [Ant98] we apply DPET to three pieces of software systems - libg++, galib and groff. The results of comparison are provided and discussed below.

\subsubsection{The Results of Case Study-2}

\begin{tabular}{|c|c|c|c|}
\hline & Libg++ & Galib & groff \\
\hline $\mathrm{LOC}$ & 10680 & 12556 & 26677 \\
\hline Classes & 55 & 69 & 290 \\
\hline Operations & 348 & 655 & 2549 \\
\hline Aggregations & 22 & 29 & 61 \\
\hline Associations & 4 & 4 & 0 \\
\hline Inneritances & 16 & 42 & 91 \\
\hline Using & 66 & 168 & 243 \\
\hline Delegations & 1 & 10 & 16 \\
\hline \multicolumn{4}{|c|}{$\begin{array}{l}\text { Remarks: } \\
\text { * The relationships among classes are kind of different from the ones defined by UML. } \\
\text { From the above table we can see that there are no associations in "groff" code. Here } \\
\text { association means cyclic aggregation or cyclic "using" relationships. No association } \\
\text { indicates that there are no cyclic aggregation and cyclic using relationships. }\end{array}$} \\
\hline
\end{tabular}

\section{Table 6.3 Code characteristics of three pieces of software}

Table 6.3 shows the extraction results of design information from the three software systems. The LOC for the three pieces of software are $10,680,12,556$ and 26,677 respectively. The number of classes is 55,69 and 290 , and the number of operations are 
348, 655 and 2,549 respectively. The different relationships (aggregations, associations, etc.) are also shown in the table.

Table 6.4 shows the results of pattern detection using our tool DPET. There are 10 adapter pattern instances detected in galib, 1 adapter pattern instance detected in libg++, 12 adapter pattern instances as well as 1 Proxy pattern instance detected in LQNS. No pattern instances are detected in groff.

\begin{tabular}{|l|l|l|l|l|l|l|l|l|}
\hline Code & \multicolumn{2}{|c|}{ Galib } & \multicolumn{2}{c|}{ Libg++ } & \multicolumn{2}{c|}{ Groff } & \multicolumn{2}{c|}{ LQNS } \\
\hline Pattern & PI & Real & PI & Real & PI & Real & PI & Real \\
\hline Adapter & 10 & 6 & 1 & 1 & 3 & 0 & 12 & 8 \\
\hline Proxy & 0 & 0 & 0 & 0 & 0 & 0 & 1 & 1 \\
\hline Bridge & 0 & 0 & 0 & 0 & 0 & 0 & 0 & 0 \\
\hline Composite & 0 & 0 & 0 & 0 & 0 & 0 & 0 & 0 \\
\hline Decorator & 0 & 0 & 0 & 0 & 0 & 0 & 0 & 0 \\
\hline $\begin{array}{l}\text { Notes: } \\
\text { PI-Pattern Instances retrieved by the tool DPET. } \\
\text { Real- Real Patterns in source code. . }\end{array}$ \\
\hline
\end{tabular}

Table 6.4 Results of pattern instance detection using our tool DPET

Table 6.5 shows the results of the extraction of design patterns using the approach proposed by Antoniol. 33 adapter pattern instances are detected in galib. There is 1 adapter pattern instance detected in libg++. In groff, 4 adapter pattern instances are detected.

\begin{tabular}{|l|l|l|l|l|l|l|}
\hline \multicolumn{1}{|r|}{ Code } & \multicolumn{2}{|c|}{ Galib } & \multicolumn{2}{r|}{ Libg++ } & \multicolumn{2}{c|}{ Groff } \\
\hline Pattern & PI & Real & PI & Real & PI & Real \\
\hline Adapter & 33 & 6 & 1 & 1 & 4 & 0 \\
\hline Proxy & 0 & 0 & 0 & 0 & 0 & 0 \\
\hline Bridge & 0 & 0 & 0 & 0 & 0 & 0 \\
\hline Composite & 0 & 0 & 0 & 0 & 0 & 0 \\
\hline
\end{tabular}




\begin{tabular}{|l|l|l|l|l|l|l|}
\hline Decorator & 0 & 0 & 0 & 0 & 0 & 0 \\
\hline Notes: \\
PI-Pattern Instances retrieved by the approach proposed by Antoniol et al. \\
Real- Real Patterns in source code.
\end{tabular}

Table 6.5 Results of pattern detection using the approach proposed by Antoniol.

Table 6.6 shows the comparison of the pattern instances extracted from the three pieces of software below.

\begin{tabular}{|c|c|c|c|c|c|c|c|c|c|}
\hline Code & \multicolumn{3}{|c|}{ Galib } & \multicolumn{3}{|c|}{ Libg++ } & \multicolumn{3}{|c|}{ Groff } \\
\hline Pattern & $\begin{array}{l}\text { PI by } \\
\text { MsA }\end{array}$ & $\begin{array}{l}\text { PI by } \\
\text { DPET }\end{array}$ & Real & $\begin{array}{l}\text { PI by } \\
\text { MsA }\end{array}$ & $\begin{array}{l}\text { PI by } \\
\text { DPET }\end{array}$ & Real & $\begin{array}{l}\text { PI by } \\
\text { MsA }\end{array}$ & $\begin{array}{l}\text { PI by } \\
\text { DPET }\end{array}$ & Real \\
\hline Adapter & 33 & 10 & 6 & 1 & 1 & 1 & 4 & 3 & 0 \\
\hline Proxy & 0 & 0 & 0 & 0 & 0 & 0 & 0 & 0 & 0 \\
\hline Bridge & 0 & 0 & 0 & 0 & 0 & 0 & 0 & 0 & 0 \\
\hline Composite & 0 & 0 & 0 & 0 & 0 & 0 & 0 & 0 & 0 \\
\hline Decorator & 0 & 0 & 0 & 0 & 0 & 0 & $\overline{0}$ & 0 & 0 \\
\hline \multicolumn{10}{|l|}{ *Precision } \\
\hline \multicolumn{2}{|l|}{ Precision } & \multicolumn{2}{|l|}{ Galib } & \multicolumn{2}{|c|}{ Libg++ } & \multicolumn{3}{|c|}{ Groff } & \\
\hline \multicolumn{2}{|l|}{ DPET } & \multicolumn{2}{|l|}{$60 \%$} & \multicolumn{2}{|l|}{$100 \%$} & \multicolumn{3}{|c|}{3 false positives } & \\
\hline \multicolumn{2}{|l|}{ MsA } & \multicolumn{2}{|l|}{$18 \%$} & \multicolumn{2}{|l|}{$100 \%$} & \multicolumn{3}{|c|}{4 false positives } & \\
\hline
\end{tabular}

Notes:

PI-Pattern Instance

MsA-the tool using Multi-stage Approach proposed by Antoniol in [AFC98].

DPET- the tool "Design Pattern Extraction Tool".

PI by DPET - the number of pattern instances retrieved by DPET.

PI by MsA - the number of pattern instances retrieved by MsA in [AFC98].

*Precision is the ratio of the number of relevant items retrieved over the total number of all items retrieved.

Table 6.6 Comparison of pattern instances obtained by DPET and MsA 


\subsubsection{Discussion on Case Study-2}

From Table 6.6, we can see that the precision has been increased from $18 \%$ to $60 \%$ when retrieving pattern instances from galib source code using DPET. Because the libg++ has only one Adapter pattern, so the precision of both DPET and MsA reaches $100 \%$. For groff code there are no real patterns enclosed in the source code. DPET only has three false positives, one less than four in MsA. Thus it seems that our approach could reduce the number of false positives and augments the precision of extraction of design patterns. So far it is hard to find a piece of source code that contains rich patterns in the reality so that we can apply it to validate our approach and obtain more reasonable and convictive precision of detection.

In groff the reason for 3 false positives is that the three pattern instances have the same static structure as the model of Adapter patterns, but the developer did not use them as adapter patterns. If we add a constraint, which in fact is the operation of delegator that has the same name as that of the operation of delegate these false positives will be eliminated. But in fact, it is very likely for developers to use different operation names of delegator and delegatee when implementing adapter design patterns. If we add this constraint in our model such adapter patterns with different operation name of delegator and delegatee will be lost. This has greater negative impact on program understanding than not using this constraint.

\subsection{Relationship between Detection Times}

In the whole process of extraction the detection time of each piece of code is shown in table 6.7 . 


\begin{tabular}{|c|c|c|c|c|}
\hline & Libg++ & galib & LQNS & groff \\
\hline LOC & 10680 & 12556 & 21182 & 26677 \\
\hline Classes & 55 & 69 & 279 & 290 \\
\hline Operations & 348 & 655 & 1911 & 2549 \\
\hline Aggregations & 22 & 29 & 142 & 61 \\
\hline Associations & 4 & 4 & 16 & 0 \\
\hline Inheritances & 16 & 42 & 193 & 91 \\
\hline Using & 66 & 168 & 700 & 243 \\
\hline Delegations & 1 & 10 & 28 & 81 \\
\hline $\begin{array}{c}* \text { DPET } \\
\text { Time(sec) }\end{array}$ & 25 & 64 & 103 & \\
\hline
\end{tabular}

Table 6.7 Code characteristics and detection time of each software system

From the Table 6.7 we can see that the detection time is increasing with the LOC (Lines of Code) increasing. But the comparison of groff and LQNS is an exception. Groff has 26677 lines of code, greater than 21182 lines of code in LQNS, but the detection time of groff $(81 \mathrm{sec})$ is less than that of LQNS $(103 \mathrm{sec})$. This may be due to the fact that relationships among classes in LQNS are much more complicated than those of groff. We try to investigate this claim in table 6.8. For instance, LQNS has 142 aggregation relationships, 193 inheritance and 700 using relationships while groff only has 61, 91, and 243 respectively. So DPET has to take more time to analyze these relationships.

In order to further explore the relationship between relationships among classes and detection time we try to remove the detection of each relationship to see what happens. The results are in the Table 6.8. The operating environment for table 6.7 and the suppositions for table 6.7 and 6.8 are given below in section 6.4 .1 . 


\begin{tabular}{|l|l|l|l|l|l|l|l|l|}
\hline & \multicolumn{2}{|c|}{ libg++ } & \multicolumn{2}{c|}{ galib } & \multicolumn{2}{c|}{ LQNS } & \multicolumn{2}{c|}{ groff } \\
\hline $\begin{array}{l}\text { Normal } \\
(\mathrm{Sec})\end{array}$ & 25 & *Rate & 64 & *Rate & 103 & *Rate & 81 & *Rate \\
\hline $\begin{array}{l}\text { Removing Delegation } \\
(\text { Sec) }\end{array}$ & 24 & $4 \%$ & 63 & $1 \%$ & 102 & $1 \%$ & 80 & $1 \%$ \\
\hline $\begin{array}{l}\text { Removing Association } \\
(\text { Sec) }\end{array}$ & 23 & $8 \%$ & 62 & $3 \%$ & 99 & $4 \%$ & 78 & $4 \%$ \\
\hline $\begin{array}{l}\text { Removing Using } \\
\text { Relationship (Sec) }\end{array}$ & 23 & $8 \%$ & 60 & $6 \%$ & 93 & $10 \%$ & 75 & $7 \%$ \\
\hline $\begin{array}{l}\text { Removing Inheritance } \\
\text { (Sec) }\end{array}$ & 24 & $4 \%$ & 63 & $1 \%$ & 102 & $1 \%$ & 80 & $1 \%$ \\
\hline $\begin{array}{l}\text { Removing Aggregation } \\
\text { (Sec) }\end{array}$ & 23 & $8 \%$ & 62 & $3 \%$ & 102 & $1 \%$ & 79 & $2 \%$ \\
\hline
\end{tabular}

Normal: the detection time spent in Table 6.7.

Removing $\mathbf{X}$ : the detection time spent when not detecting $\mathbf{X}$ relationship.

*Rate: the decreasing rate of the detection time after not detecting $\mathbf{X}$ in comparison with the normal detection time, i.e., Rate $=($ NDT - RDT $) /$ NDT, where

$\mathrm{NDT}=$ Normal detection time; $\mathrm{RDT}=$ the detection time spent when not detecting some relationship.

- Sampling steps for detection time:

The program runs five times in each case so that five sets of time values are obtained, and then get the average values.

Table 6.8 Detection time table after not detecting some relationships

The operating environment of our case study is shown in Table 6.9 for reference and comparison in the future.

\begin{tabular}{|l|l|}
\hline Operating Environment: Sun Workstation Sun Blade 100 \\
\hline Operating System & SunOS 5.8 \\
\hline Physical Memory & $128 \mathrm{M}$ DIMM \\
\hline Processor & $500-\mathrm{MHz}$ UltraSPARC[tm]-Iie, 64-bit \\
\hline Cache Memory & $256-\mathrm{KB} \mathrm{L2}$ external cache \\
\hline Disk & $15 \mathrm{~GB}$ \\
\hline
\end{tabular}

Table 6.9 Operating environment of this experiment 


\subsubsection{Discussion - Hypotheses on design information detection time}

- Hypothesis 1: After removing the detection of using relationships the detection time of LQNS relatively decreases by $10 \%$ from 103 to 93 . Though the LOC of Groff is greater than that of LQNS the number of using relationships of LQNS is almost 3 times as that of Groff (243/700). The using relationships may be a contributing factor to the detection time of LQNS compared to that of groff.

- Hypothesis 2: The Lines of Code of galib is 1.2 times that of libg ++ but the detection time of galib is 2.7 times compared to libg++. This might imply that the detection time is related with not only LOC, but also the number of some kind of relationships among classes in the software systems.

- Hypothesis 3: In each piece of code the number of using relationships is the greatest among a variety of relationships. From table 6.8 we can see that the rate of the detection time changing has been decreased after removing the detection of a using relationship. In comparison with removing the detection of other relationships, using relationships have a great impact on the detection time. On the other hand, the number of delegation relationships is the second least in each piece of code in Table 6.7, removing it has little impact on the detection time. The reason why removing using relationships has the greatest impact on the detection time is because the number of using relationships in each system is the most numerous of all kinds of relationships.

- Hypothesis 4: After removing the detection of association relationship its impact on the detection time seems greater than that of removing delegations, even though the number of association relationships is less than that of delegation relationships. This is because the definition of association is cyclic aggregation and using relationships. 


\section{Chapter 7 Conclusions \& Future Work}

This chapter discusses important conclusions that are discussed in this thesis and presents some suggestions for future research.

This thesis presents a rule-based approach in the extraction of design patterns in order to understand software systems at a pattern level. The models for formal descriptions of design patterns proposed are based on static and dynamic characteristics of design patterns. It is stored in a rule base, while design information extracted from source code is stored in the fact base, design patterns are extracted by querying the knowledge base composed of both the fact and rule base.

\subsection{Conclusions}

Four pieces of software systems have been applied in our case studies. Their results are encouraging and show that DPET can effectively extract design patterns from source code. Furthermore, by comparing these results with the approach proposed by Antoniol et al, our approach does reduce the false instances and augment the precision of pattern detection. We also carried out a study on the relationship between detection time, and class relationships and proposed some hypotheses.

The following conclusions are made from this thesis:

- The rule-based approach makes implementation simple, flexible and extendable. This is the most important contribution of this thesis.

- The model for formal descriptions of design patterns is based on static and dynamic characteristics of design patterns. For some behavioral design patterns dynamic information is the only way to identify them. 
- Logic-based pattern detection makes this process language-independent. The pattern detection can be applied to other language like Java without modifications.

- Separation of the extraction of design information from the design pattern recovery makes the whole extraction process more effective and overcomes the drawbacks of logic programming - slow execution time.

- The parser we have developed is capable of scanning the entire $\mathrm{C}++$ source code so that some important relationships like delegation can be detected thereby contributing to the effective detection of design patterns.

- The software maintainers can get benefits from DPET performing between automatic and manual mode.

- The case studies on a large-sized software system have shown that our approach can be applied to large-sized software systems.

- In addition, the intermediate results (i.e. fact base) could be used to visualize the class diagram representing the static information of the system under review (cf. Appendix D)

\subsection{Future Work}

Several issues in which we are still interested in our future work are as follows:

- An approach to automatic instrumentation for obtaining dynamic information is needed to study further. We have tried to get dynamic information of software systems using the profiling technique. Profiling can provide call graphs and performance information in crucial section of the source code. But there is no information of call sequence available from call graph, which is the 
key to extracting some behavioral design patterns based on dynamic information. Profiling alone cannot make significant contributions to the extraction of design patterns. Hence, an automatic instrumentation technique is needed for obtaining the call sequence. The basic technique of program instrumentation is to instrument a code at points of interest in the program. During the execution the instrumented code is then executed together with the original program code. It provides the time stamp of each call from which we can get the call sequence by comparing the call graph generated from the profiling tool. Manual instrumentation has very limited application. For largesized software with several hundred of classes, it would become impossible.

- A graphic interface is needed to display class diagrams of software systems based on the fact base obtained by DPET. In addition, a set of classes composing a design pattern should be showed in certain way like highlight line so as to facilitate the process of reverse engineering. This can give software maintainers an intuitive view of software systems at pattern and design level.

- Further performance analysis is needed to prove the hypotheses presented in section 6.4. In terms of the data from our case studies several hypotheses about the relationship between detection time and class relationships are proposed. They need to be fully proved in the future. 
In conclusion, this thesis has presented a comprehensive and simple approach to design pattern detection in order to aid program understanding and software maintenance. We believe that the software maintainers as well software engineers will find this approach useful. 


\section{References}

[Ale79] Alexander, C.; "The Timeless Way of Building”. Oxford University Press, 1979

[Ant98] Antoniol, G.; Fiutem, R.; and Cristoforetti, L.; "Design pattern recovery in object-oriented software. " In 6th International Workshop on Program Comprehension, 153-160, Ischia, Italy, June 1998.

[Ant03] ANTLR applications. http://www.antlr.org/showcase.html Last modified: 20/01/2002; date visited: 30/04/2003.

[App00] Appleton, B.; "Patterns and Software: Essential concepts and terminology". http://www.enteract.com/ bradapp/docs/patterns-intro.html Last modified 02/14/2000, Date visited 30/11/2002.

[Ban98] Bansiya, J.; "Automating Design-Pattern Identification." Dr. Dobb's Journal, June 1998

[Bec96] Beck, K.; Crocker, R.; Meszaros, G.; Coplien, J.O.; Dominick, L.; Paulisch, F.; Vlissides, J.; "Industrial experience with design patterns." Software Engineering, Proceedings of the 18th International Conference on , 25-29, Page(s): $103-114$, Mar 1996

[Ben92] Bennett K.; Bull, T.; Yang, H.; “A Transformation System for Maintenance - Turning Theory into Practice", Proceeding of the international Conference on Software Maintenance, IEEE Computer Society Press, pp. 146-155,1992.

[Boo99] Booch, G.; Rumbaugh, J; and Jacobson, I. "The Unified Modeling Language User Guide". Addison-Wesley, Reading, MA, 1999.

[Boo94] Booch, G.; "Object-Oriented Design with Application." Addison-Wesley, 1994

[Bro96] Brown, K.; "Design Reverse-engineering and Automated Design Pattern Detection in Smalltalk",1996

[Bus96] Buschmann, F.; Meunier, R.; Rohnert, H.; Sommerlad, P., and Stal, M.; "Pattern-Oriented Software Architecture: A System of Patterns." John Wiley \& Sons, New York, April,1996 
[Bus94] Buss, E et al.; “ Investigating Reverse Engineering Technologies for the CAS Program Understanding Project”, IBM Systems Journal, 33(3), pp.477-500,1994

[Cag00] Cagnin, M.I.; Penteado, R.; Braga, R.T.V.; Masiero, P.C.; "Reengineering using design patterns. " Reverse Engineering, Proceedings. Seventh Working Conference on , Page(s): $118-127,2000$

[Cha98] Chase, P. M.; Christey, M. S.; Harris, R. D.; Yeh, S. A.; “Managing Recovered Function and Structure of Legacy Software Components", Proceedings of the $5^{\text {th }}$ Working Conference on Reverse Engineering, IEEE Computer Society Press, pp. 79-88,1998.

[Che97] Cheng, B.; Jeng, J.; “ Reusing Analogous Components”, IEEE Transactions on Knowledge and data Engineering, 9(2), pp. 341-349, MarApr 1997

[Che94] Cheng, B.; "Applying Formal Methods in Automated software development", Journal of Computer and software engineering, 2,pp. 137 164, Feb. 1994

[Chi90] Chikofsky,E.J. and Cross II, J.H.; "Reverse Engineering and Design Recovery: A taxonomy. " IEEE Software 7,1, 13-17, Jan.1990

[Cin00] Cinneide, M.O.; "Automated refactoring to introduce design patterns." Software Engineering. Proceedings of the 2000 International Conference on, Page(s): $722-724,2000$

[Coo98] Cooper, W. James; "The Design patterns Java Companion,” http://www.patterndepot.com/put/8/JavaPatterns.htm, 1998, Date visited: $5 / 12 / 2002$

[Cop92] Coplien, O. J.; “Advanced C++: Programming Styles and Idioms." Reading,Mass.:Addison-Wesley, 1992

[Ede98] Eden, H. A.; Hirshfeld, Y.; Yehudai, A.; “LePUS - A Declarative Pattern Specification Language”. Technical report 326/98, department of Computer Science, Tel Aviv University, 1998 
[Ede99] Eden, H. A.; Gil, J.; Hirshfeld, Y.; and Yehudai, A.; "Towards a Mathematical foundation for Design Patterns". Technical Report 1999004, Department of Information Technology, Uppsala University, 1999.

[Ede97] Eden, H. A.; Gil, J. and Yehudai, A.; "Precise specification and automatic application of design patterns".In Proceedings of the Twelfth IEEE International Automated Software Engineering Conference Ne ada, Nov 1997.

[Fra99] Roy Gregory Franks, “Performance Analysis of Distributed Server Systems", phD thesis, Deptment of Systems and Computer Engineering, Carleton University, OCIECE-00-01, Dec 20, 1999

[Fra96] Greg Franks, "Layered Queueing Network Solver Software Design”, Department of Systems and Computer Engineering, Carleon University, 1996

[Gam95] Gamma, E.; Johnson, R.; and Helm, R.; and Vlissides, J.; “Design Patterns: Elements of Reusable Object-Oriented Software." AddisonWesley,1995.

[Ga 96] Gannod, C. G.; Cheng, B., "Strongest Post-Condition Semantics as a Basis for Reverse Engineering", Journal of Automated Software Engineering, 3, 1/2, pp. 139-164, July 1996

[Gan96] Gannod, C. G.; Cheng, B.; "Using Informal and Formal Techniques for the Reverse Engineering of C Programs", Proceedings of the Third Working Conference on Reverse Engineering, IEEE Computer Society Press, pp. 249-258,1996

[Gpr02] GNU Gprof, http://www.gnu.org/manual/gprof-2.9.1/gprof.html, Last Modified: 07/11/1998; Date Visited: 12/07/02.

[Har90] Harband, J.; " SEELA: Maintenance and Documenting by ReverseEngineering", Proceedings of the international Conference on Softweare Maintenance, IEEE Computer Society, p. 146,1990

[Hon01] Hong, M.; Xie, T.; Yang, F.;" JBOORET: an automated tool to recover OO design and source models." Computer Software and Applications 
Conference, 2001. COMPSAC 2001. 25th Annual International, Page(s): $71-76,2001$

[Jer97] Jerding, D.;S. Rugaber; “ Using Visualization for Architectural Localization and Extraction", Processings of the Fourth Working Conference on Reverse Engineering, IEEE Computer Society Press, pp. 56-65, 1997

[Kel99] Keller, K.R; Schauer R.;Robitaille, S.; and Page, P; "Pattern-based Reverse Engineering of Design Components." In Proc. of the 21th International Conference on Software Engineering, Los Angeles, USA, page 226-235. IEEE Computer Society Press, May 1999.

[Kra96] Kramer, C.; Prechelt, L. "Design recovery by automated search for structural design patterns in object-oriented software." In Proc. Of the $3^{\text {rd }}$ working Conference on Reverse Engineering(WCRE), Monterey,CA, page 208-215. IEEE Computer Society Press, November 1996.

[Lan95] Lange, B. D.; Nakamura, Y.; "Interactive Visualization of Design Patterns Can Help in Framework Understanding", In Proceedings Of the10th Annual Conference on Object-Oriented Programming Systems, Languages, and Applications (OOPSLA -95), ACM Press, pp. 342-357. 1995

[Lit01] Littlefair, Tim; "An investigation into the use of software code metrics in the industrial software development environment", PHD thesis, At the Faculty of Communications, Health and Science, Edith Cowan University, Mount Lawley Campus, 18 June 2001

[Lu 00] Lu, Chih-Wei ; Chu, William C.; Chang, Chih-Hung; Chuang, Yeh-Ching; Liu, Xiaodong; and Yang, Hongji; "Reverse Engineering”. Department of Information Engineering, Feng Chia University, Taichung, Taiwan, 2000.

[Me 01] Mens K.; Michiels I.; Wuyts, R.; "Supporting Software Development through Declaratively Codified Programming Patterns", 2001

[Men01] Mens, T.; Tourwe, T.; "A Declarative Evolution Framework for ObjectOriented Design Patterns". IEEE, 2001 
[Mu198] Muller, H. A.; "Rigi User's Manual Version 5.4.4," Department of Computer Science University of Victoria, 1998.

http://www.rigi.csc.uvic.cal, Last Modified: October 23, 2000, Date visited: Aug 2002.

[My194] Mylopoulos, J.; et al., "Towards an Integrated Toolset for Program Understanding”, Proceedings of the 1994 IBM CAS Conference (CASCON '94), pp. 19-31, 1994

[Nie02] Niere, J.; Schafer, W.; Wadsack, B. J.; Wendehals L.; "Towards PatternBased Design Recovery." University of Paderborn, Department of Math and Computer Science, Germany, 2002

[Par93] Terence John Parr, "Language Translation Using PCCTS and C++, A Reference Guide". Automata Publishing Company, 1993

[Pre94] Pree, W.; "Design Patterns for Object-Oriented Software Development." Reading, Mass.: Addison-Wesley, 1994

[Pro03] http://sources.redhat.com/binutils/docs2.12/gprof.info/Introduction.html\#Introduction , Last modified 2002-0307, Date visited 12/07/02

[Rat99] Rational Software Corporation, "OMG Unified Modeling Language Specification Version 1.3", June. 1999

[Raj90] Rajlich, V.; Damaskinos, N.; "Algorithm for Graphic Layout in VIFOR", Proceedings of the International Conference on Software Maintenance, IEEE Computer Society Press, pp. 142-145, 1990

[Rea00] Reasoning5 CBMS, Reasoning, Systems Inc. On URL http://www.reasoning.com/, Date visited Sep. 2002

[Ric99] Richner, T.; Ducasse, S.; "Recovering high-level views of object-oriented applications from static and dynamic information." Software Maintenance (ICSM '99) Proceedings. IEEE International Conference on , Page(s): 13 22, 1999

[Rie96] Riehle, D; Zullighoven, H.; "Understanding and using patterns in software development". Theory and Practice of Object Systems,2(1):313,1996 
[Ros99] Rosenberg, J.; Lennox, J; Schulzrinne, H; “ Programming Internet Telephony Services", IEEE Internet Computing,3(3), pp. 63-72,May/June 1999

[Rum91] Rumbaugh, J.; Blaha, M.; Premerlani, W.; Eddy, F.; and Lorensen, W.; “Object-Oriented Modeling and Design." Prentice-Hall, Englewood Cliffs, NJ, 1991.

[Sch99] Schauer, R. and Keller, R. K. Pattern visualization for software comprehension. In 6th International Workshop on Program Comprehension (Ischia, Italy, June 1998), 153-160.

[See98] Seemann, J.; Gudenberg, W. J.; "Pattern-Based Design Recovery of Java Software." ACM SIGSOFT Software Engineering Notes,23(6), ACM Press, November 1998.

[Shu96] Shull, F.; Melo, L. W.; and Basili, R.V.; "An inductive method for discovering design patterns from object-oriented software systems." Technical report, University of Maryland, Computer Science Department, College Park, MD, 20742 USA, 1996.

[Sys00] Systa, T.; Yu, P.; Muller, H.; “ Analyzing Java Software by Combining Metrics and Program Visualization," Proceedings of the $4^{\text {th }}$ European Conference on Software Maintenance and Reengineering, IEEE Computer Society ,pp. 199-208, 2000

[Tes00] Tesanovic, A.; “ What is a pattern?”. Linkoping University, Linkoping, Sweden, 2000

[Ton99] Tonella, P. and Antoniol, G.; "Object Oriented Design Pattern Inference." In Proc. of the $5^{\text {th }}$ Symposium on Software Development Environments(SDE5), pages 230-238. IEEE Computer Society Press, September 1999.

[Uml03] http://pigseye.kennesaw.edu/ dbraun/csis4650/A\&D/UML tutorial, Date visited Jan 2003 
[Wil93] Wills, M. L; “Flexible Control for Program Recognition”, Proceedings of the $1^{\text {st }}$ Working Conference on Reverse Engineering, IEEE Computer Science Press, pp. 134-143,1993

[Wuy01] Wuyts, R.; “ A Logic Meta-Programming Approach to Support CoEvolution of Object-Oriented Design and Implementation". $\mathrm{PhD}$ thesis, Department of Computer Science, Vrije University Brussel, Belgium, January 2001

[Wuy98] Wuyts, R.; "Declarative reasoning about the structure of object-oriented systems". In Proceedings of TOOLS USA 1998, pages 112-124, IEEE Computer Society Press, 1998 


\section{Appendix A The Detailed Extraction Process of Proxy and Adapter Patterns}

\section{A.1 Extraction Description of Proxy design patterns}

\section{Parsing the code using $\mathrm{C}++$ parser}

The following sample code fragment is adopted from the "Proxy" sample code in [Gam95].

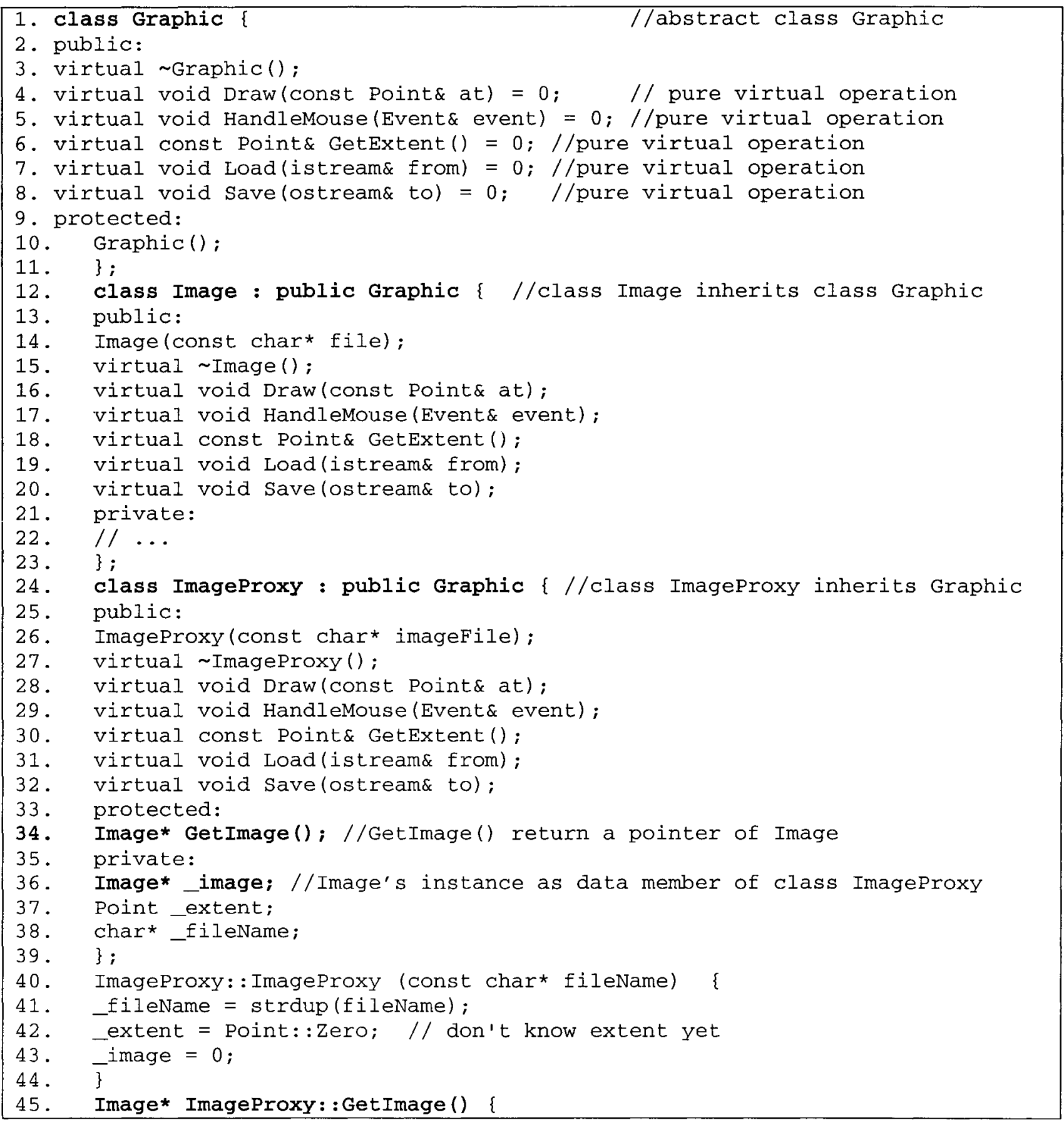




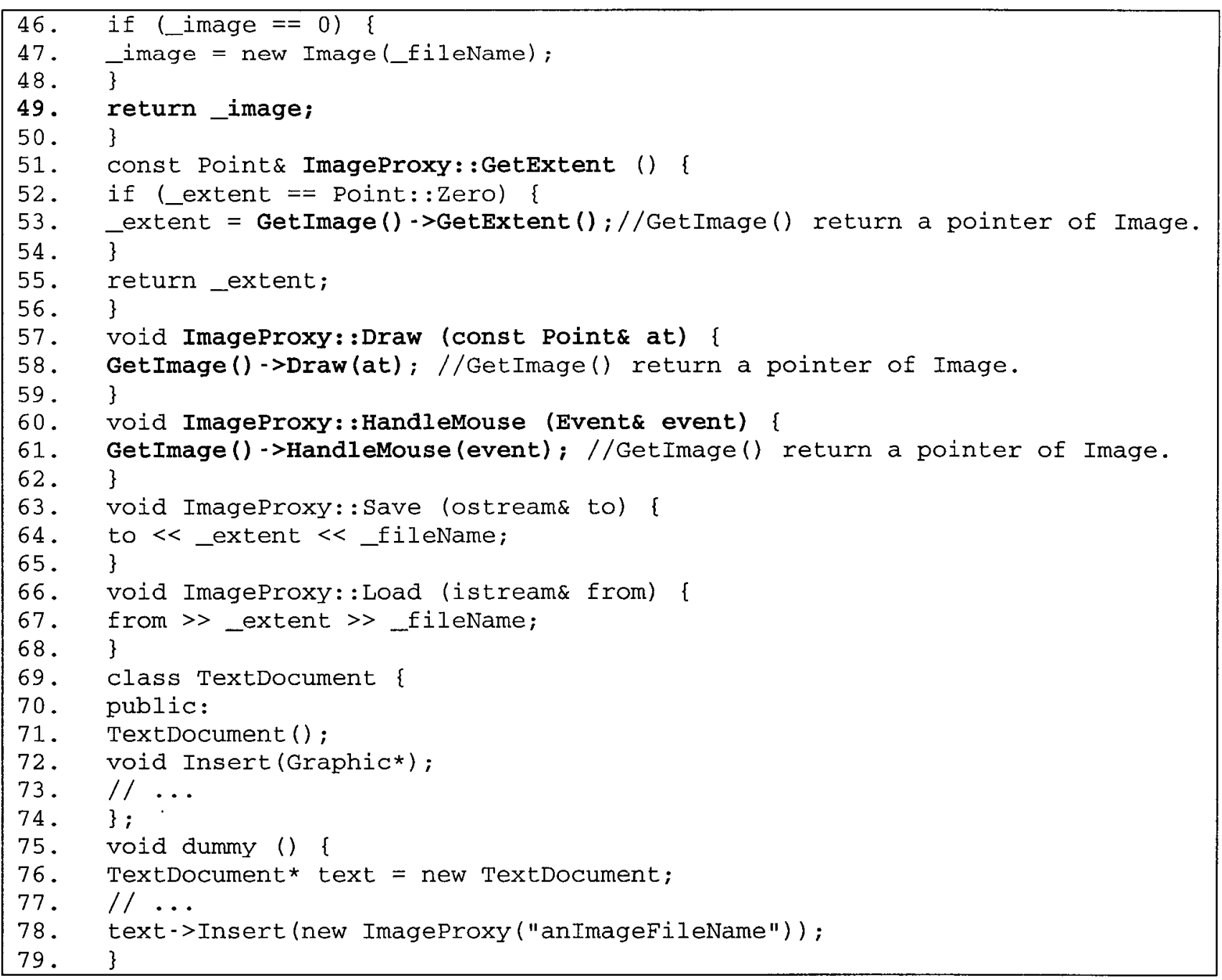

Comments:

The lines that are related with pattern detection in code have been bolded with comments. In order to better understand them, all comments are put together as follows for giving a whole view of the static information we need to extract from code.

// class Image is abstract class

// Draw() is pure virtual operation

// HandleMouse () is pure virtual operation

$/ /$ GetExtent() is pure virtual operation

$/ /$ Load() is pure virtual operation

//Save() is pure virtual operation

// class Image inherits class Graphic

//class ImageProxy inherits class Graphic

// GetImage() of class ImageProxy return a pointer of Image

// Image's instance as data member of class ImageProxy

// GetImage() return a pointer of Image.

$/ /$ So the above should be equivalent of extent = _image->GetExtent () ;

// ImagePoxy delegates Image via GetExtent() operation

// Draw() returns a pointer of Image. 
// So the above should be equivalent of extent = _image- $>$ Draw () ;

// ImagePoxy delegates Image via Draw() operation

$/ /$ GetImage() return a pointer of Image.

// So the above should be equivalent of extent = _image- $>$ HandleMouse () ;

// ImagePoxy delegates Image via HandleMouse() operation.

The above static information is exactly what we use.

\section{Storing the program information in "cccc.db" after parsing the code}

- Data format for Classes:

CCCC_Module@ModuleName@ModuleType

CCCC_ModExt@ModuleName@ModuleType@

FileName@Line@Definition/Declaration@Properties@Metrics

CCCC_Module@Graphic@class@

CCCC_ModExt@Graphic@Class@proxy.C@78@definition@F??T2@LOC:4 COM:6 MVG:0@2@d@

CCCC_Module@Image@class@

CCCC_ModExt@Image@class@proxy.C@7@definition@F??T2@LOC:3 COM:0 MVG:0@2@d@

CCCC_ModExt@Image@class@proxy.C@14@declaration@F??T2@LOC:1 COM:2 MVG:0@2@D@

CCCC_ModExt@Image@Class@proxy.C@97@definition@F??T0@LOC:4 COM:3 MVG:0@0@d@

CCCC_Module@ImageProxy@class@

CCCC_ModExt@ImageProxy@class@proxy.C@117@definition@F??T0@LOC:8 COM:2

MVG:0@0@d@

- Data format for Operations:

CCCC_Member@ModuleName@OperationName@ReturnType@Parameter@

CCCC_MemExt@ModuleName@OperationName@ReturnType@Parameter@

FileName@Line@Declaration/Definition@OperationType@Metrics

CCCC_Member@Graphic@Draw@void@(const Point\&at)@

CCCC_MemExt@Graphic@Draw@void@(const Point

\&at)@proxy.C@83@declaration@F??T0@LOC:1 COM:2 MVG:0@0@D@

CCCC_Member@Graphic@HandleMouse@void@(Event \&event)@

CCCC_MemExt@Graphic@HandleMouse@void@(Event\&event)@proxy.C@84@declaration@F??T0

@LOC: 1 COM:0 MVG:0@0@D@

CCCC_Member@Image@Draw@void@(const Point \&)@

CCCC_MemExt@Image@Draw@void@(const Point\&)@proxy.C@9@declaration@F??T0@LOC:1

COM:0 MVG:0@0@D@

CCCC_Member@Image@Draw@void@(const Point \&at)@

CCCC_MemExt@Image@Draw@void@(const Point

\&at)@proxy.C@103@declaration@F??T0@LOC:1 COM:2 MVG:0@0@D@

CCCC_Member@Image@HandleMouse@void@(Event \&event)@

CCCC_MemExt@Image@HandleMouse@void@(Event

\&event) @proxy.C@104@declaration@F??T0@LOC:1 COM:0 MVG:0@0@D@

CCCC_Member@ImageProxy@Draw@void@(const Point \&at)@

CCCC_MemExt@ImageProxy@Draw@void@(const Point

sat)@proxy.C@123@declaration@F??T0@LOC:1 COM:2 MVG:0@0@D@

CCCC_MemExt@ImageProxy@Draw@void@(const Point

sat)@proxy.C@163@definition@F??T?@LOC:3 COM:2 MVG:0@?@d@

CCCC_Member@ImageProxy@HandleMouse@void@(Event \&event)@

CCCC_MemExt@ImageProxy@HandleMouse@void@(Event

sevent)@proxy.C@124@declaration@F??T0@LOC:1 COM:0 MVG:0@0@D@

CCCC_MemExt@ImageProxy@HandleMouse@void@(Event

sevent)@proxy.C@168@definition@F??T?@LOC:3 COM:2 MVG:0@?@d@

- Data format for basic relationships among classes (pass by reference, has by value, inheritance) and class attribute: data member in class. 
CCCC_UseRel@ModuleName1@ModuleName2@(Pointer/Reference)@,

CCCC_UseExt@ModuleName1@ModuleName2@(Pointer/Reference)

CCCC_UseRe1@Graphic@ImageProxy@@

CCCC_UseExt@Graphic@ImageProxy@@proxy.C@117@inheritance@F??T0@*@0@I@

CCCC_UseRel@Image@ImageProxy@_image@

CCCC_UseExt@Image@ImageProxy@_image@proxy.C@135@has by reference@F??T2@LOC:0

COM:0 MVG:0@2@h@

CCCC_UseRel@Graphic@Image@@

CCCC_UseExt@GraphiC@Image@@roxy.C@97@inheritance@F??T0@*@0@I@

- Data Format for Delegation Instances:

CCCC_Delegation@Reference/Pointer@CalledOperation@CallingModule@

CallingOperation@

CCCC_Delegation@GetImage@Draw@ImageProxy@Draw@

CCCC_Delegation@GetImage@GetExtent@ImageProxy@GetExtent@

CCCC_Delegation@GetImage@HandleMouse@ImageProxy@HandleMouse@

\section{Extracting static info(classes and their interrelationships) from ccc.db and storing it into "writepro.pl" (executing detect cccc.db)}

- The facts generated by running "detect cccc.db"

class (concrclass, graphic).

class (concrclass, image).

class (concrclass, imageProxy).

operation (_,_,graphic, draw, , ,, ).

operation (_'_,graphic, getExtent,_,_,_).

operation (_', graphic, handlemouse, _'_',').

operation (_, , image, draw, , , , , ) .

operation (_,-., image, getExtent,_,_,_).

operation (_,_, image, handleMouse,_,_,_).

operation (_, , imageProxy, draw, ., _, $)$.

operation (_,_, imageProxy, getExtent,_'_'_).

operation (_, , imageProxy, getImage, ,','_).

operation (_'_, imageProxy, handlemouse,_','_).

inheritance (graphic, image) .

inheritance (graphic, imageProxy).

delegation (imageProxy, image) .

- The rules for identifying the "Proxy" design pattern

proxy (Subj, RealSubj, Proxy, $N$ ) :- //N is the number of pattern instance proxy_stcrelations (Subj, Realsubj, Proxy), proxy_classes (Subj, Realsubj, Proxy), proxy_ops (Subj, Op, Realsubj, Proxy), increment $(\mathrm{N})$.

proxy_classes (Subj, RealSubj, Proxy) :-

class (_, Subj),

class (_, Realsubj),

class (_, Proxy),

!.

proxy_stcrelations (Subj, Realsubj, Proxy) :-

inheritance (Subj, RealSubj),

inheritance (Subj, Proxy),

delegation (RealSubj, Proxy).

proxy_ops (Subj, op, Realsubj, Proxy) : -

operation (_,_, Subj, Op, ,','_), 

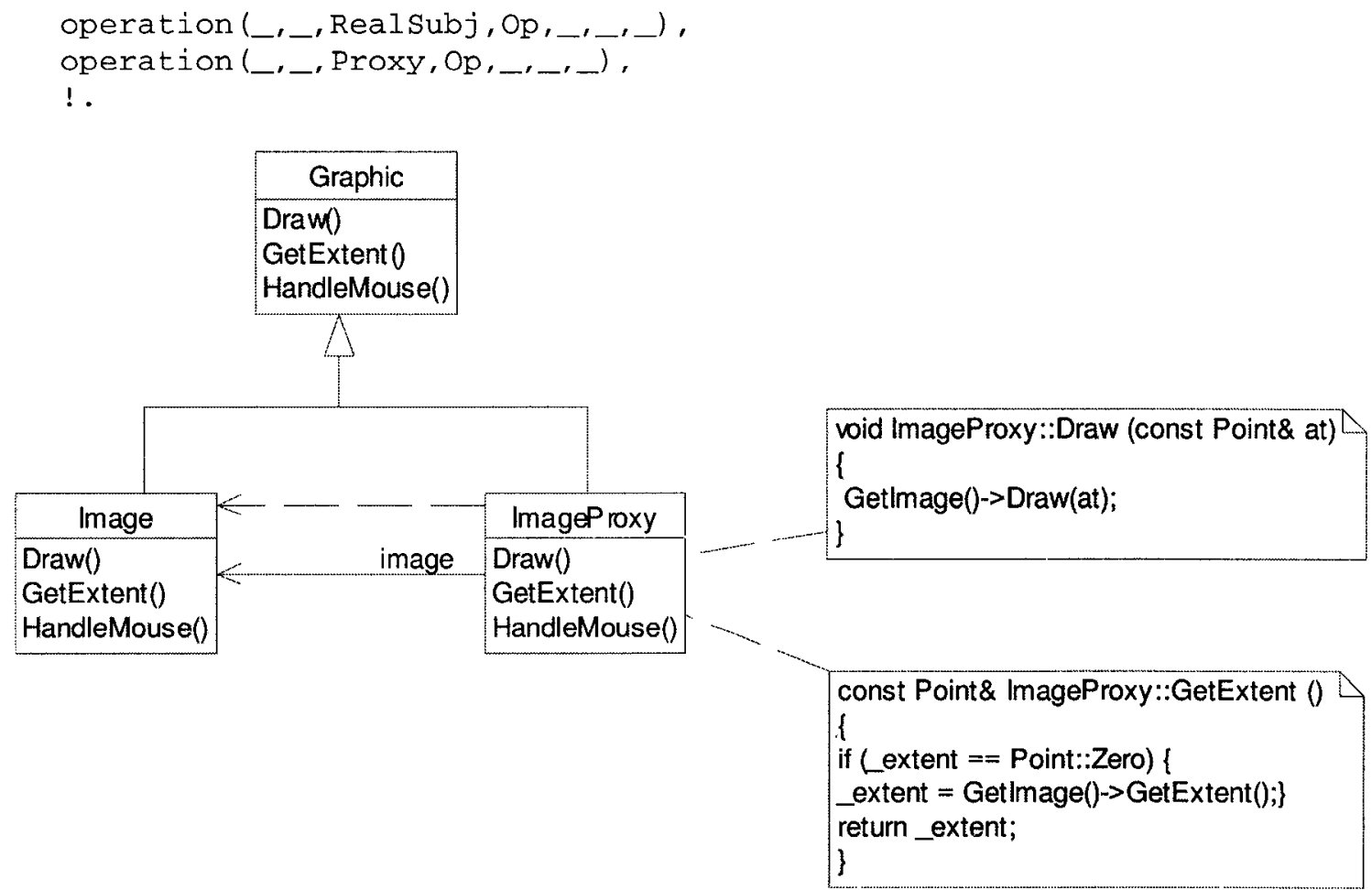

The above diagram is UML representation of the "Proxy" design pattern exiting among these three classes.

4. The results after running SWI-Prolog inference engine to identify design patterns:

Subject $=$ Graphic

Realsubject= Image

Proxy= ImageProxy 


\section{A.2 Extraction description for Adapter design patterns}

\section{Parsing source code using $\mathbf{C + +}$ parser}

The following sample code fragment is from LQNS that is applied in case study-2 later.

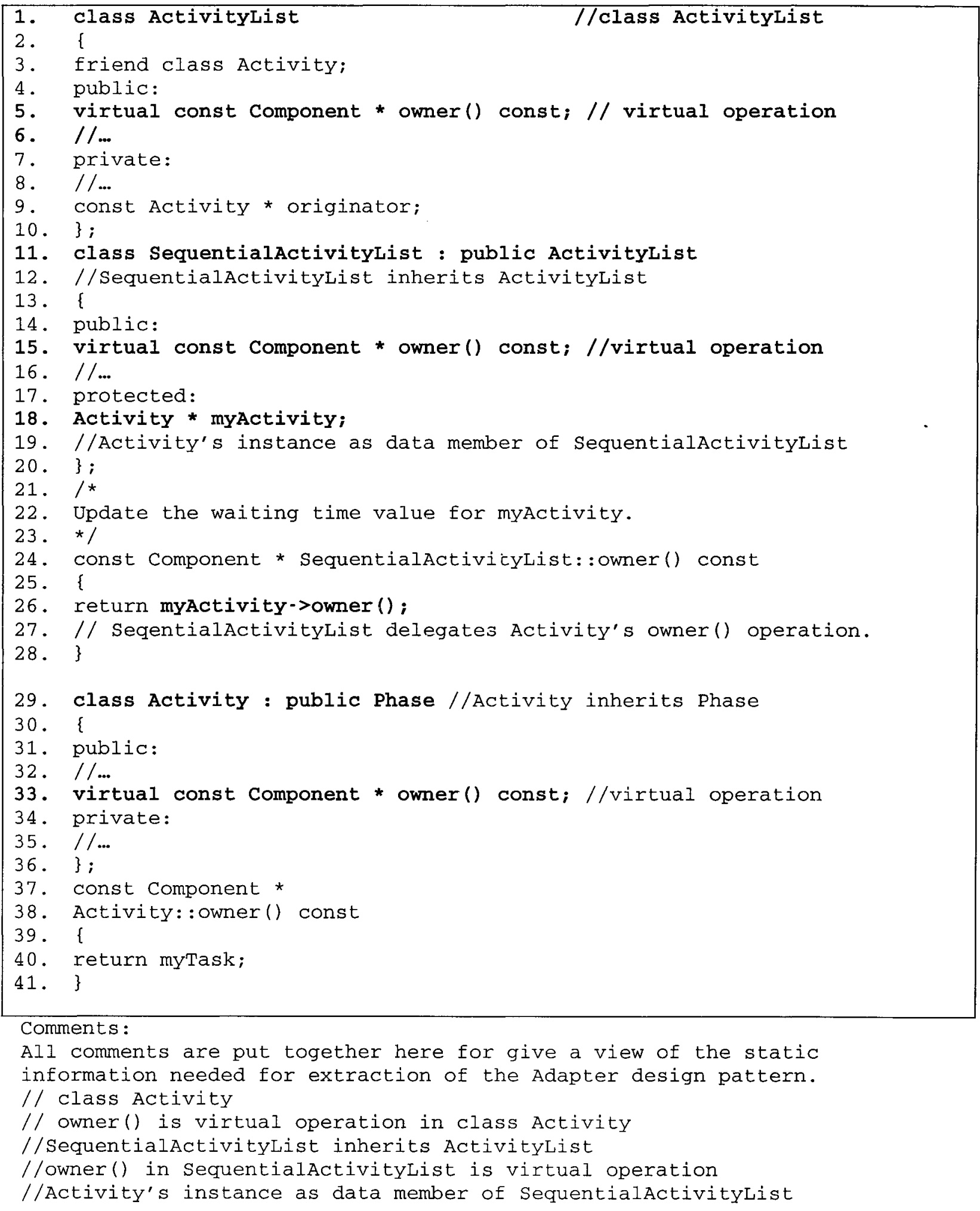


// SeqentialActivityList delegates Activity's owner() operation

/ lowner() in Activity class is virtual operation

The above information is exactly what we use to extract the Adapter design pattern.

\section{Storing program information in "cccc.db" after parsing the code}

- Data format for Classes:

CCCC_Module@ModuleName@ModuleType

CCCC_ModExt@ModuleName@ModuleType@,

FileName@Line@Definition/Declaration@Properties@Metrics

CCCC_Module@Activity@class@

CCCC_ModExt@Activity@Class@activity.h@186@definition@T??T0@LOC:16 COM:16

MVG:0@0@d@

CCCC_ModExt@Activity@class@activity.h@280@declaration@T??T2@LOC:1 COM:0

MVG:0@2@D@

CCCC_ModExt@Activity@class@phase.h@126@declaration@????2@LOC:1 COM:0

MVG:0@2@D@

CCCC_ModExt@Activity@class@task.h@114@declaration@????2@LOC: 1 COM: 0

MVG:0@2@D@

CCCC_ModExt@Activity@class@entry.h@120@declaration@????2@LOC:1 COM: 0

MVG:0@2@D@

CCCC_Module@ActivityList@class@

CCCC_ModExt@ACtivityList@Class@activity.h@166@declaration@????2@LOC:1 COM:0

MVG:0@2@D@

CCCC_ModExt@ActivityList@Class@activity.h@278@definition@T??T2@LOC:11 COM:6

MVG:0@2@d@

CCCC_ModExt@ActivityList@Class@phase.h@125@declaration@????2@LOC:1 COM:0

MVG:0@2@D@

CCCC_ModExt@ActivityList@class@task.h@116@declaration@????2@LOC:1 COM:0

MVG:002@D@

CCCC_Module@SequentialActivityList@Class@

CCCC_ModExt@SequentialActivityList@Class@activity.h@323@definition@T??T0@LOC

:7 COM:1 MVG:0@0@d@

- Data format for Operations:

CCCC_Member@ModuleName@OperationName@ReturnType@Parameter@

CCCC_MemExt@ModuleName@OperationName@ReturnType@Parameter@

FileName@Line@Declaration/Definition@OperationType@Metrics

CCCC_Member@Activity@owner@@()@

CCCC_MemExt@Activity@owner@()@activity.h@223

@declaration@T??T0@LOC:1 COM:0 MVG:0@0@D@

CCCC_MemExt@Activity@owner@@()@activity.C@421

@definition@TT???@LOC:4 COM:0 MVG:1@?@d@

CCCC_Member@ActivityList@owner@@()@

CCCC_MemExt@ACtivityList@owner@()@activity.h@296@declaration@T??T0@LOC:1

COM:0MVG:0@0@D@

CCCC_MemExt@@ACtivityList@owner@@()@activity.C@962@definition@TT???@LOC:5

COM:0 MVG:1@?@d@

CCCC_Member@SequentialActivityList@owner@@()@

CCCC_MemExt@SequentialActivityList@owner@()@activity.h@328@declaration@T??T

0@LOC: 1 COM:0 MVG:0@0@D@

CCCC_MemExt@SequentialActivityList@owner@()@activity.C@1046@definition@TT??

?@LOC:4 COM:0 MVG:1@?@d@ 
- Data format for basic relationships among classes (pass by reference, has by value, inheritance) and class attribute: data member in class.

CCCC_UseRel@ModuleName1@ModuleName2@(Pointer/Reference)@

CCCC_UseExt@ModuleName1@ModuleName2@(Pointer/Reference)

CCCC_UseRel@Activity@SequentialActivityList@myActivity@

CCCC_UseExt@Activity@SequentialActivityList@myActivity@activity.h@334@has by reference@T??T1@LOC:0 COM:0 MVG:0@1@h@

CCCC_UseRel@ActivityList@SequentialActivityList@@

CCCC_UseExt@ActivityList@SequentialActivityList@@activity.h@323@

inheritance@T??T0@*@0@I@

- Data Format for Delegation Instances:

CCCC_Delegation@Reference/Pointer@CalledOperation@CallingModule@

CallingOperation@

CCCC_Delegation@myActivity@owner@SequentialActivityList@owner@

\section{Extracting static information (classes and their interrelationships) from program} information in the file "cccc.db" and storing it into "writepro.pl" in Prolog representation.

- The facts generated by running "detect $c c c c . d b$ "

class (concrclass, activity) .

class (concrclass, activityList).

class (concrclass, sequentialActivityList).

operation (_, , activitylist, owner,_,.,_.).

operation (_,_, activity, owner,_,_,_).

operation (, , , sequentialActivitylist, owner, , , , - ).

inheritance (activityList, sequentialActivityList).

delegation (sequentialActivityList, activity) .

- The rules for identifying "Adapter" design pattern

adapter (Target, Adapter, Adaptee, N) :-

adapter_stcrelations (Target, Adapter, Adaptee),

adapter_classes (Target, Adapter, Adaptee),

adapter_ops (Adapter, Adaptee, Target, Request, SpecRequest) ,

increment $(\mathrm{N})$.

adapter_classes (Target, Adapter, Adaptee) :-

class (_, Target),

class (concrclass, Adapter),

class (concrclass, Adaptee),

!.

adapter_ops (Adapter, Adaptee, Target, Request, SpecRequest) : -

operation (_,_, Adaptee, SpecRequest, , , , $)$,

operation (_,_, Target, Request, $, 1,-$ ),

operation (_,_, Adapter, Request,_,_,_), 1 .

adapter_stcrelations (Target, Adapter, Adaptee) :-

inheritance (Target, Adapter),

delegation (Adapter, Adaptee). 


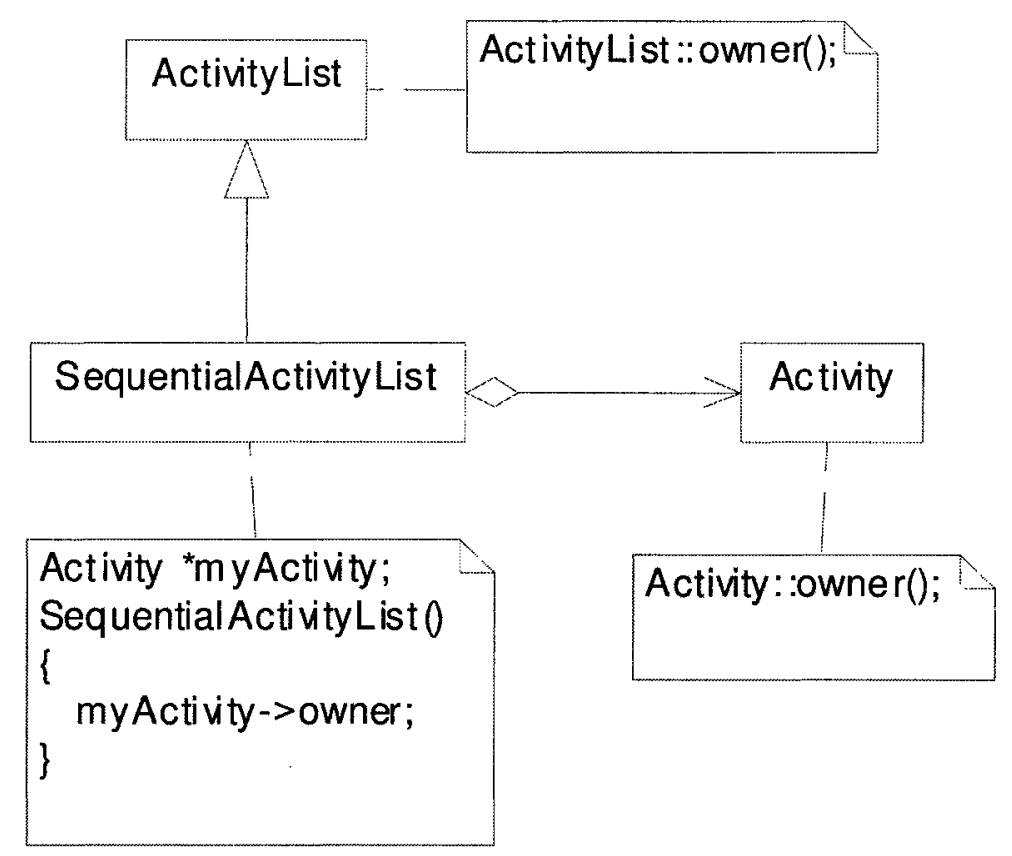

The above diagram is UML representation of "Adapter" design pattern exiting among these three classes.

4. By running SWI-Prolog inference engine to identify design patterns:

Target $=$ activityList

Adapter $=$ sequentialActivitylist

Adaptee $=$ activity 


\section{Appendix B The Data Format for Source Code Information}

The data formats used in the extraction process is shown below.

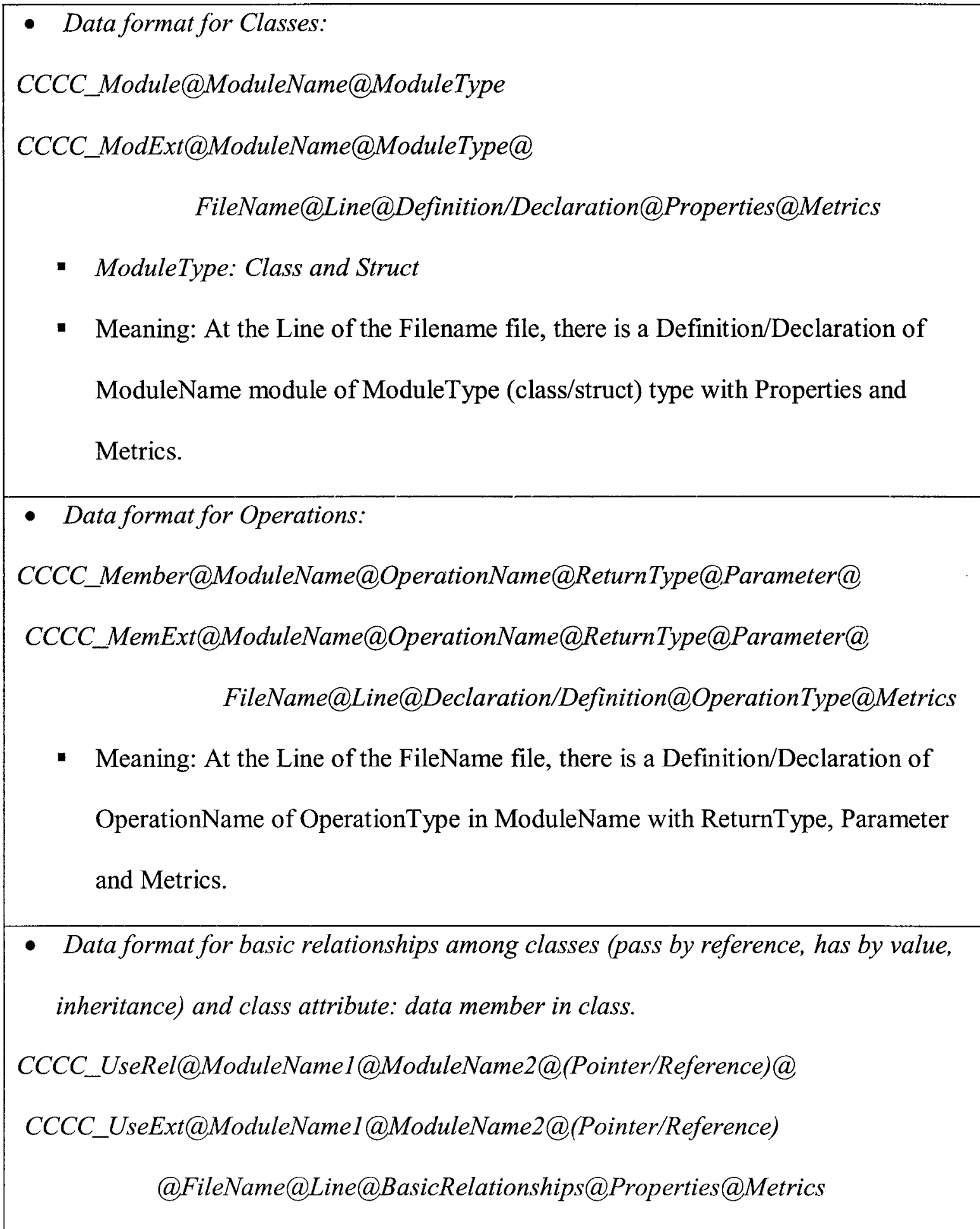


- Meaning: At the Line of the FileName file, there is a basic Relationship between ModuleName1 and ModuleName2 with Properties and Metrics.

- Data Format for Delegation Instances:

CCCC_Delegation@Reference/Pointer@CalledOperation@CallingModule@ CallingOperation@

- Meaning: CallingOperation of CallingModule invokes a CalledOperation of Object Reference/Pointer.

- Data Format for Creation Information:

CCCC_Composition@InstanceClass@InstanceObject@ScopeClass@ ScopeOperation@

- Meaning: There is an InstanceObject of type of InstanceClass created in ScopeOperation of ScopeClass.

- Data Format for ReturnType of operation:

CCCC_Return@RealClassType@ReturnPointer@ReturnClassType@,

\section{ReturnOperation@}

- Meaning: The ReturnOperation of ReturnClassType Returns a ReturnPointer or an object of RealClassType. 


\section{Appendix C The Results Of Profiling The Sample Code of Chain of Responsibility Design Patterns}

The graph below is the complete call graph obtained by profiling the sample code of

Chain of Responsibility design patterns in Section 5.3.2.

It consists of two parts. One is call graph profile and the other is flat profile.

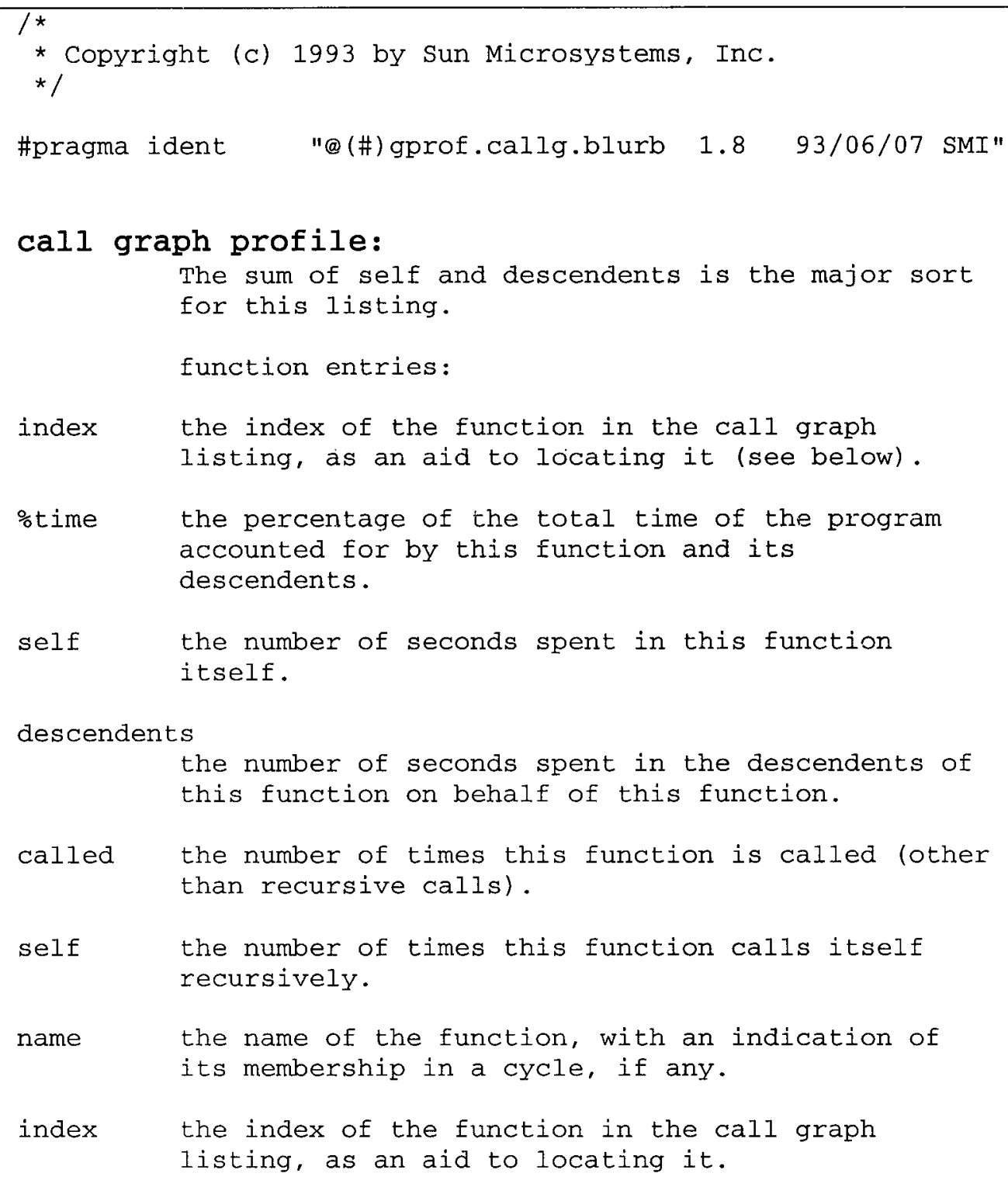




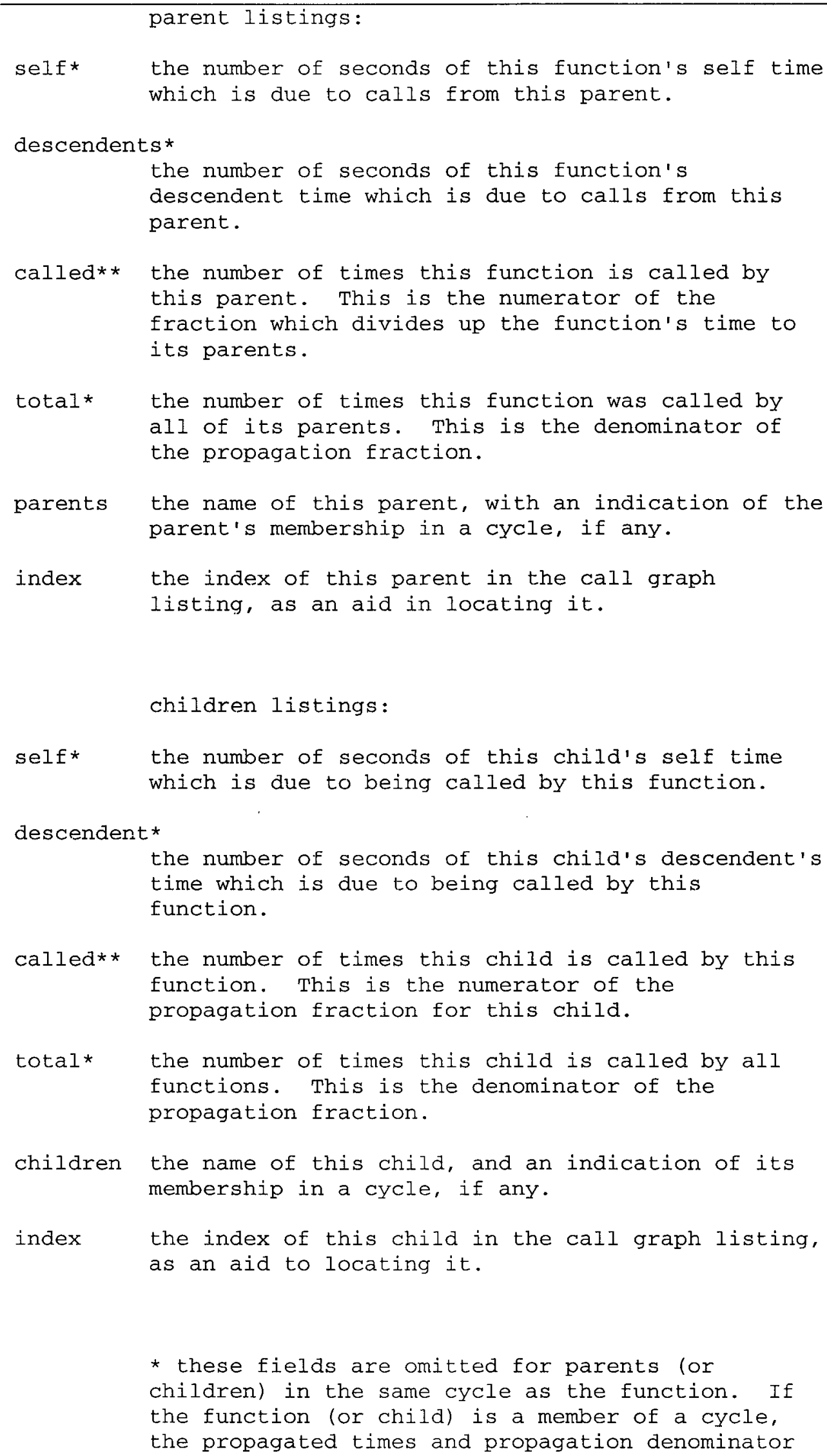


represent the self time and descendent time of the cycle as a whole.

** static-only parents and children are indicated by a call count of 0 .

cycle listings:

the cycle as a whole is listed with the same fields as a function entry. Below it are listed the members of the cycle, and their contributions to the time and call counts of the cycle.

granularity: each sample hit covers 4 byte(s) for $100.00 \%$ of 0.01 seconds

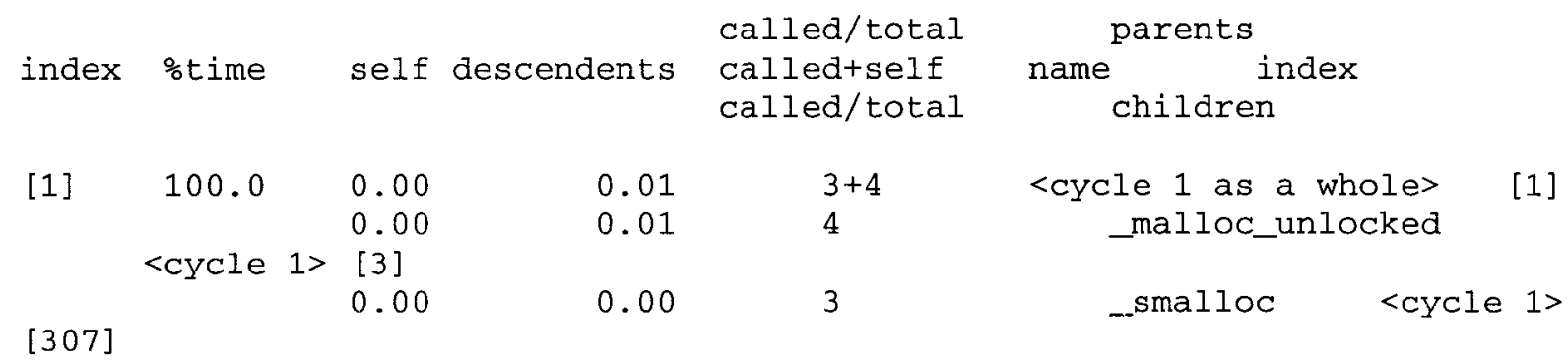

$\begin{array}{llll}100.0 & 0.00 & 0.01 & \\ \text { <cycle 1> } & 0.00 & 0.01 & 3 / 3 \\ & {[3]} & & \\ & 0.00 & 0.00 & 3 / 18 \\ & 0.00 & 0.00 & 3 / 18\end{array}$

[307]

\begin{tabular}{|c|c|c|}
\hline & 0.00 & 0.01 \\
\hline & 0.00 & 0.01 \\
\hline
\end{tabular}

[3]

$\begin{array}{lll}0.01 & 0.00 & 1 / 1 \\ 0.00 & 0.00 & 4 / 4 \\ 0.00 & 0.00 & 1 / 1 \\ & & 3\end{array}$

[307]

1

$3 / 3$

$1 / 1$

$4 / 4$

3

\author{
<spontaneous> \\ malloc [2] \\ _malloc_unlocked \\ _mutex_lock [302] \\ _mutex_unlock [303]
}




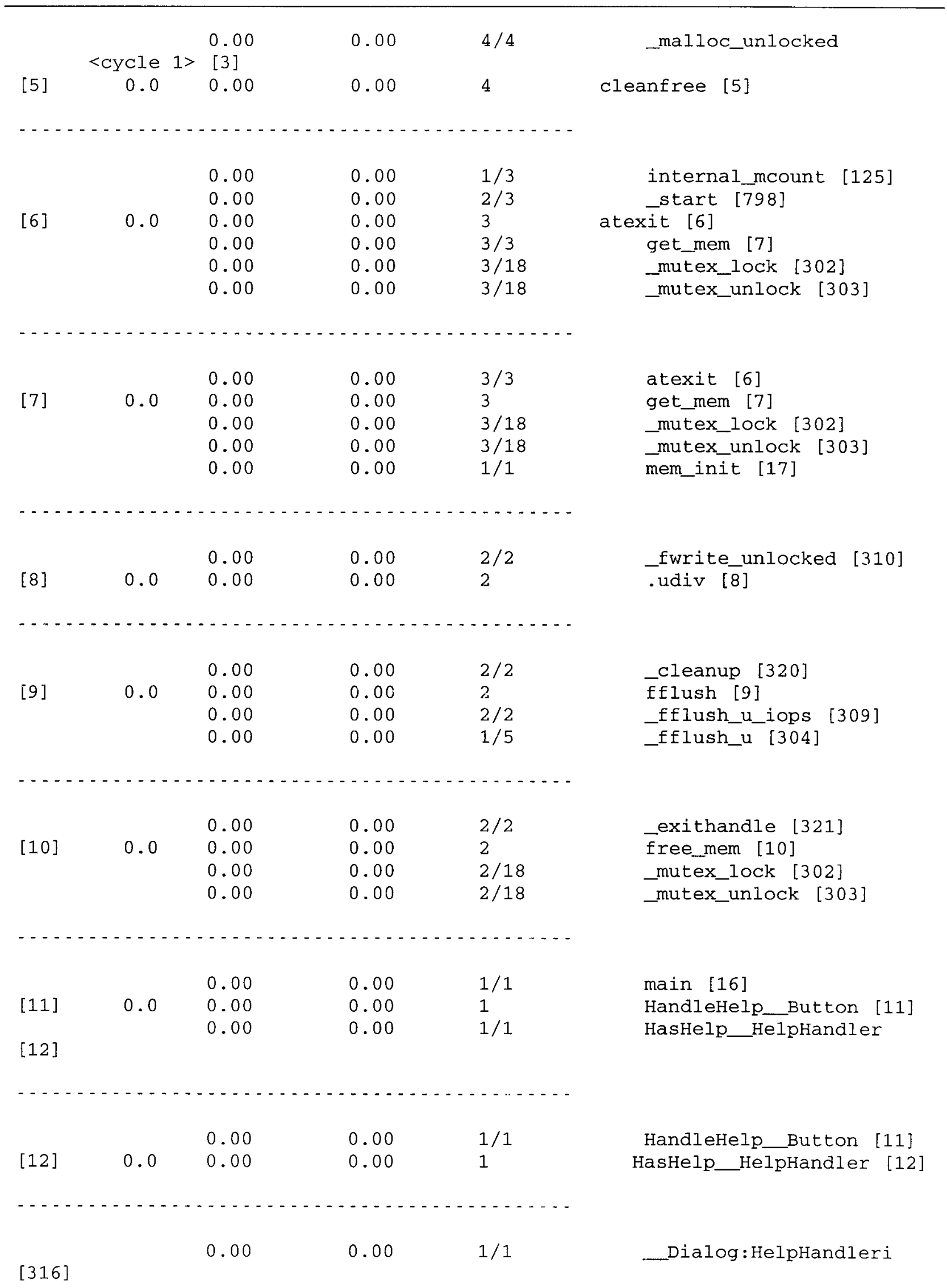




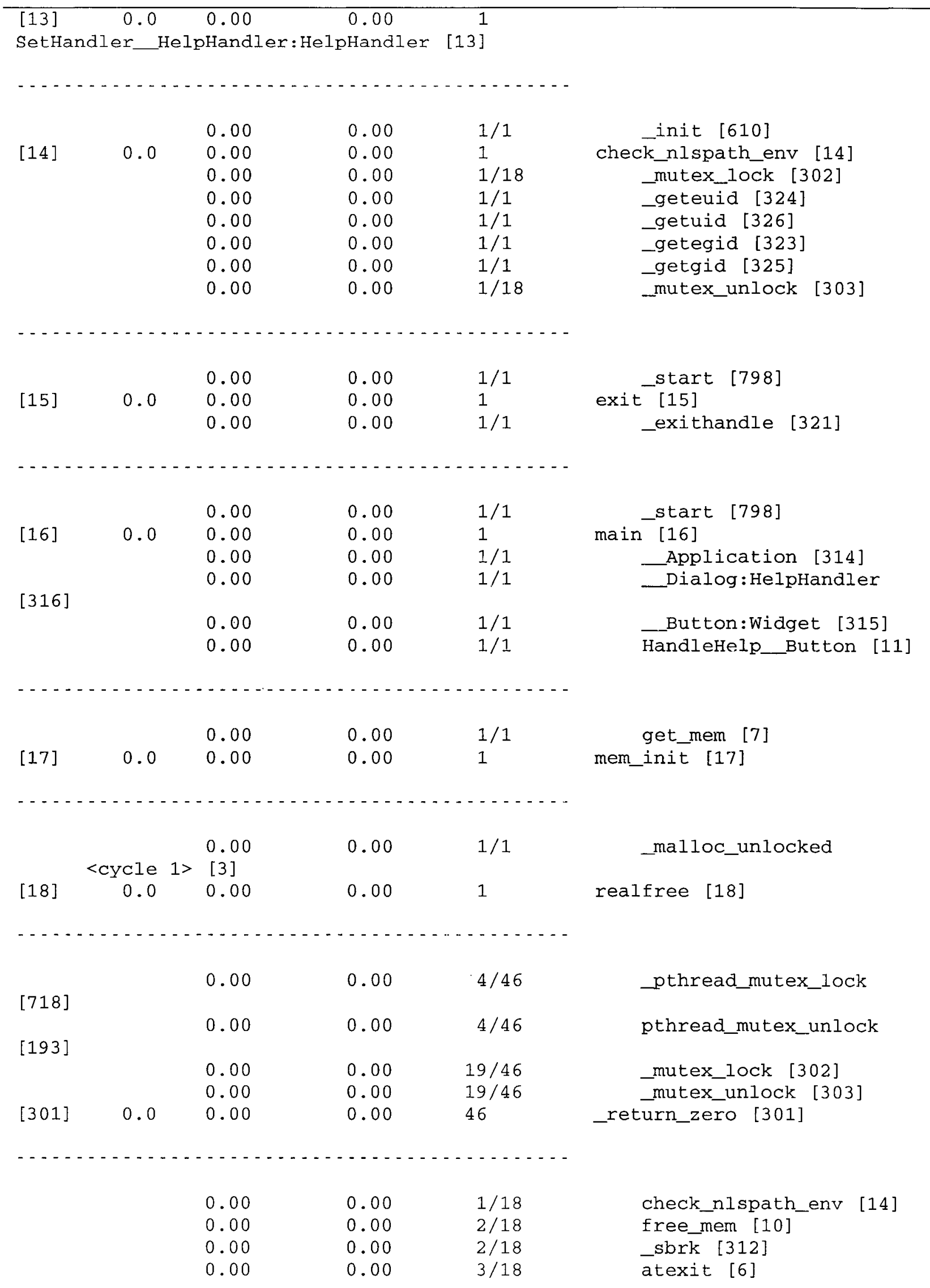




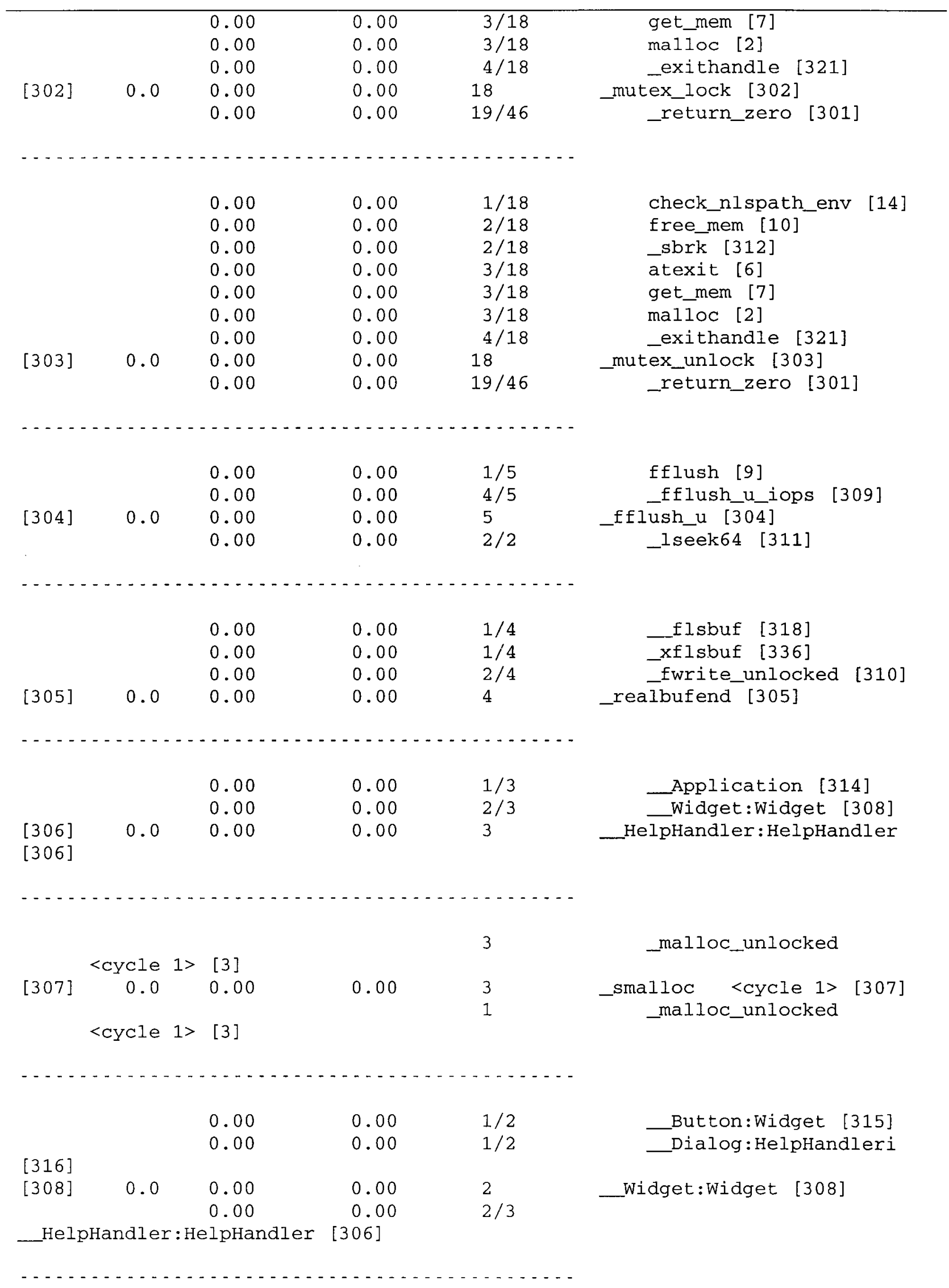




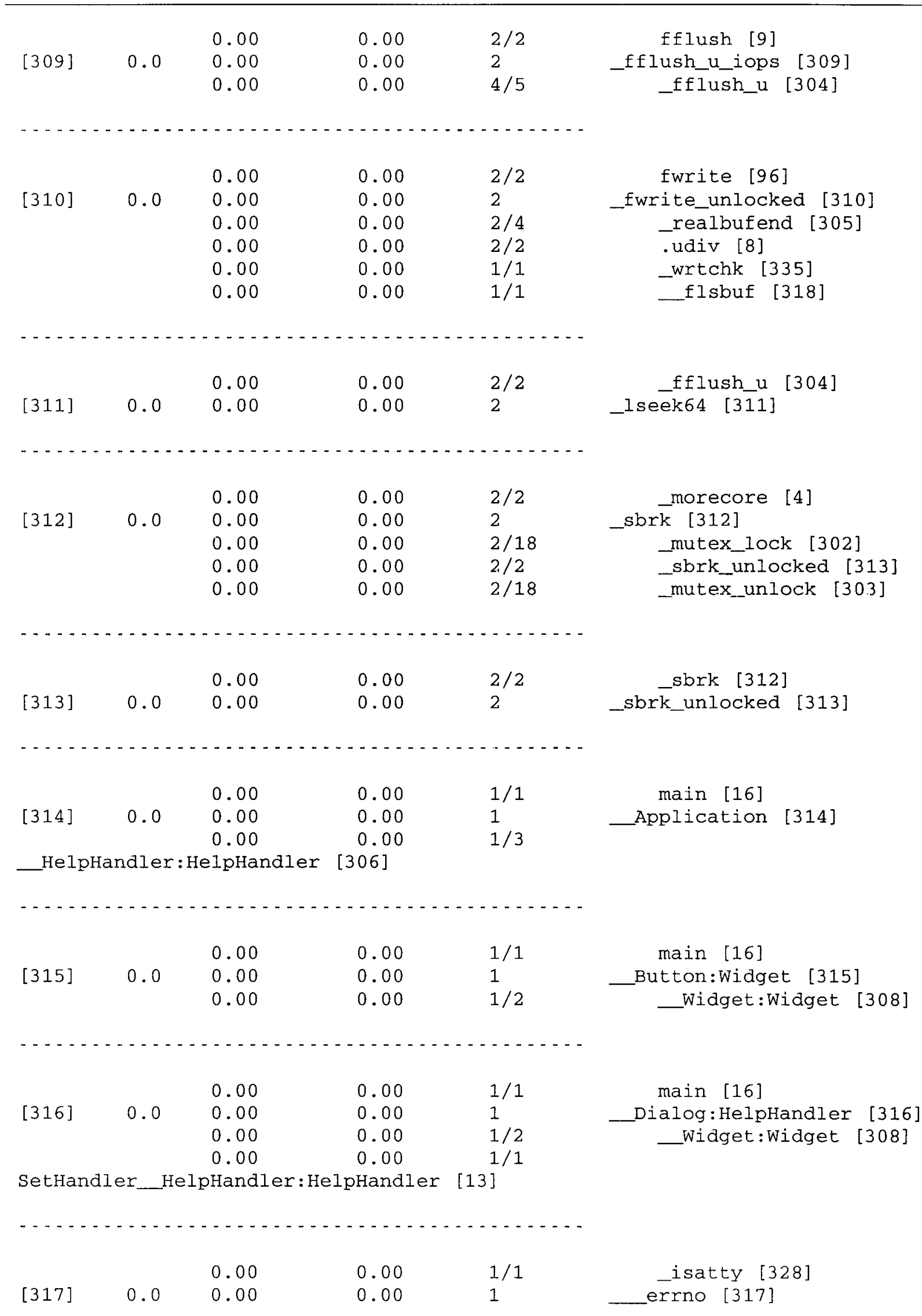




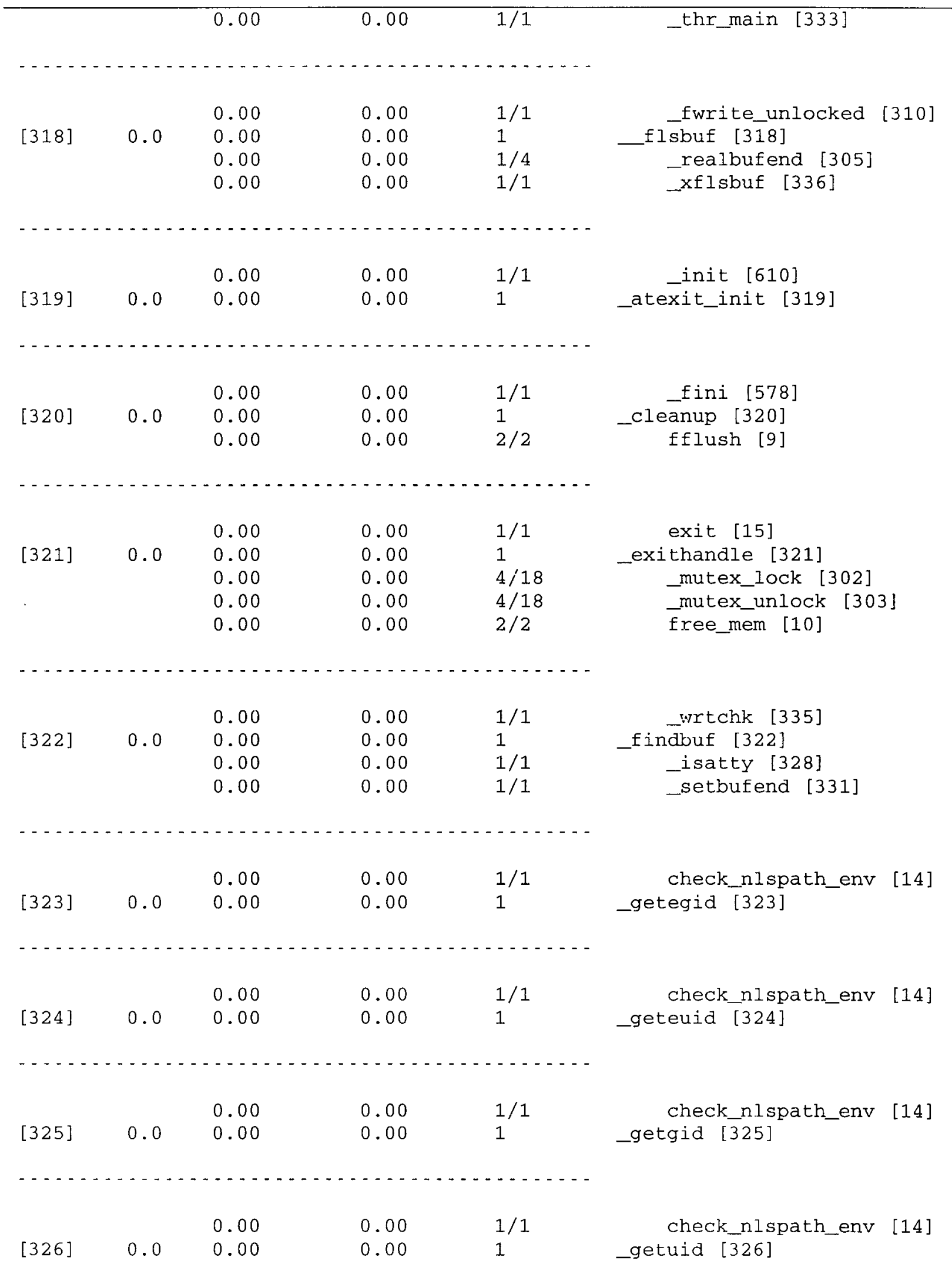




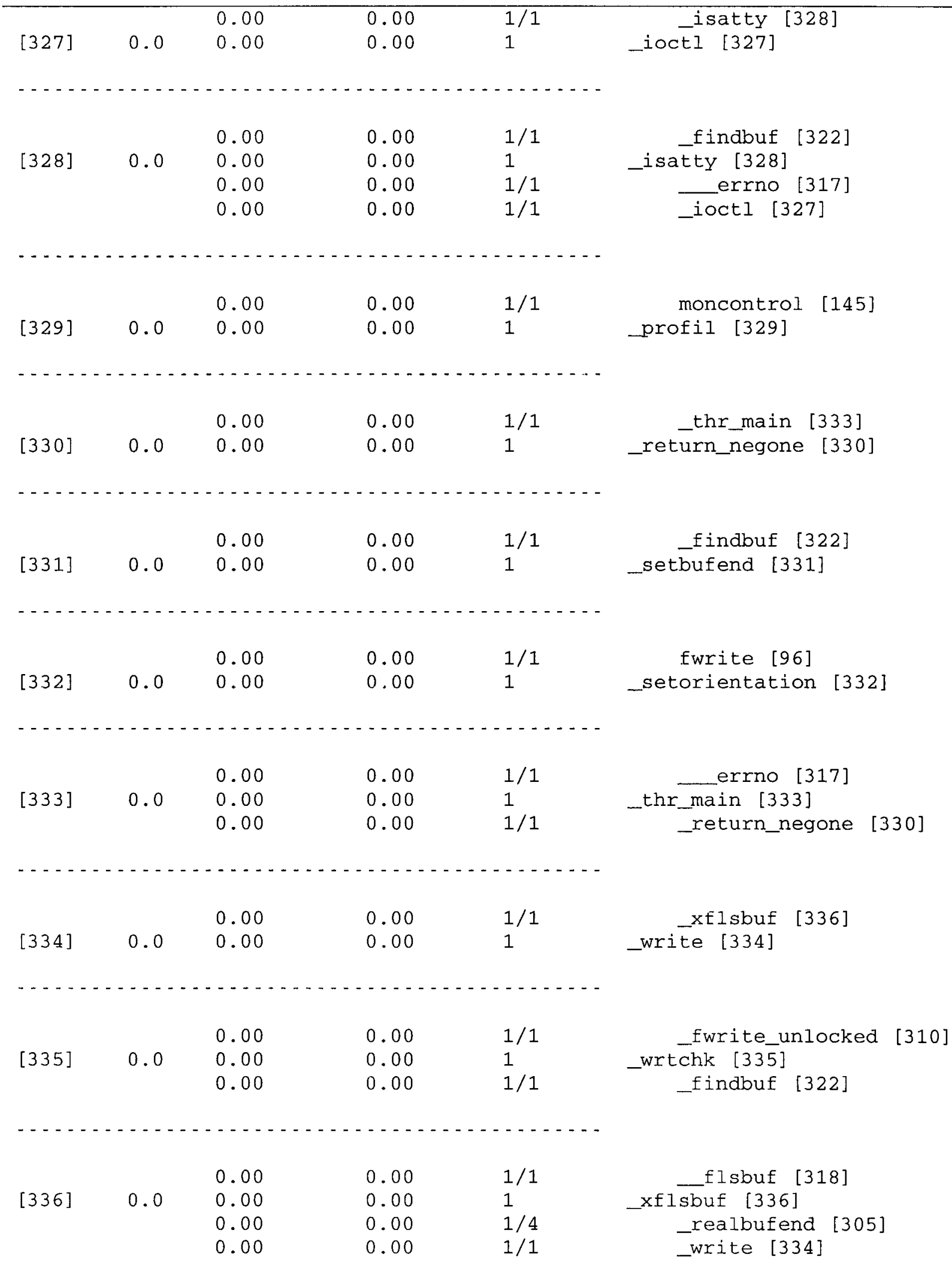




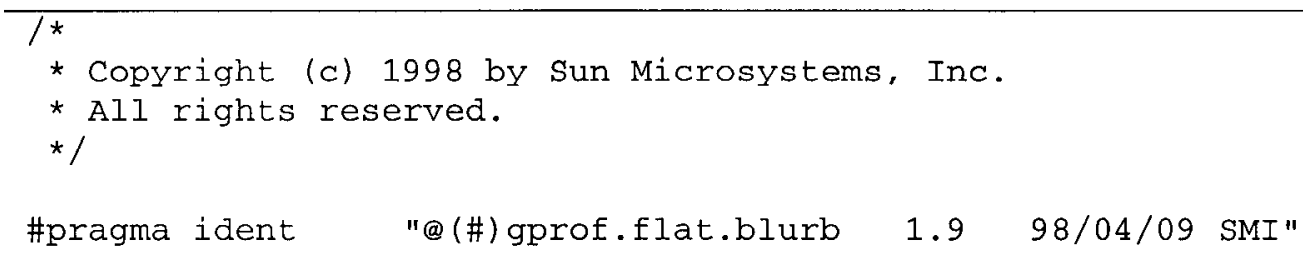

\section{flat profile:}

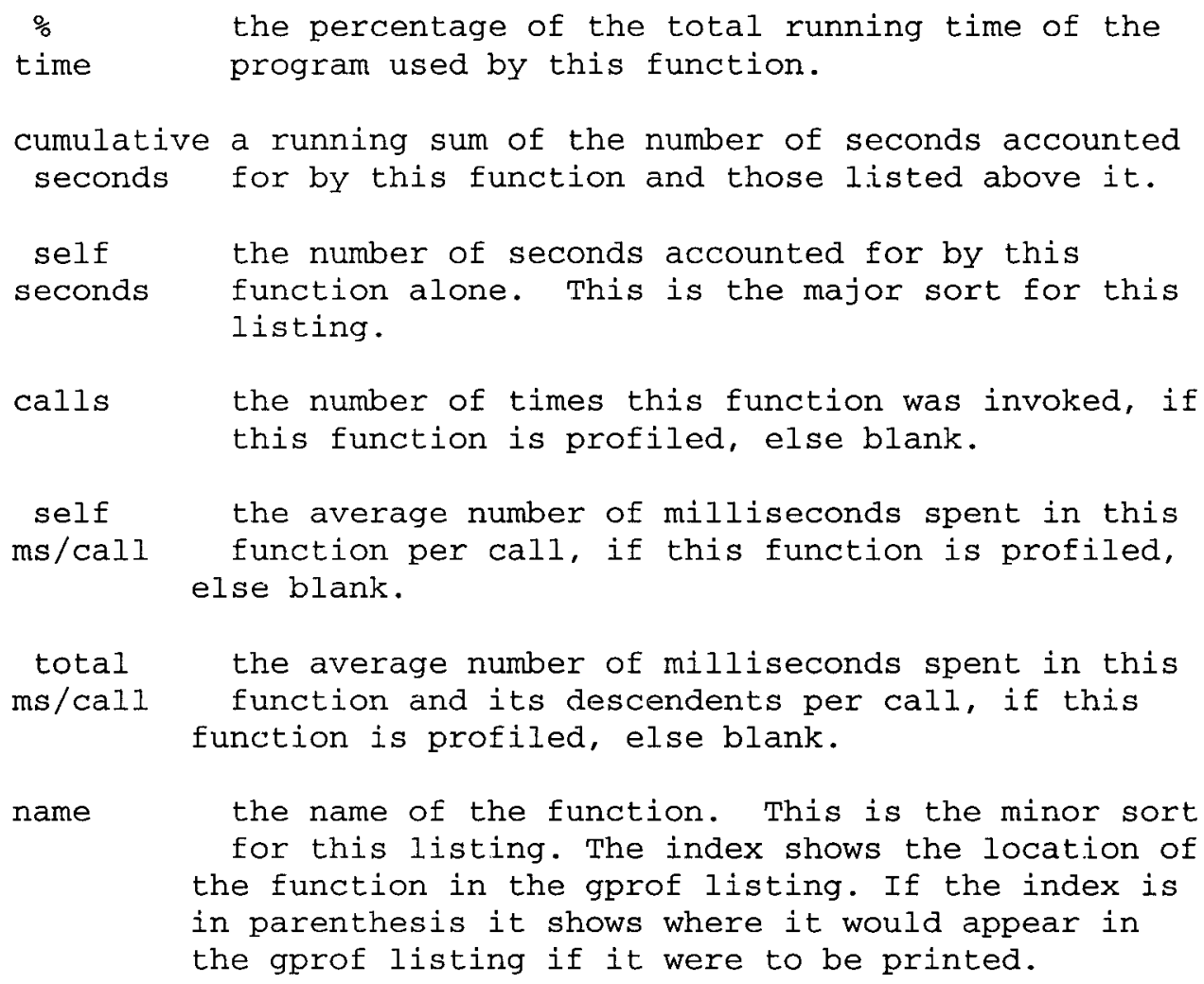

Index by function names and object modules listing:

The index entries are mapped to the function names. If the same function name appears in multiple object modules, each of these names is prefixed with a module identifier followed by a colon. The shared object modules listing that follows maps these identifiers to appropriate objects.

granularity: each sample hit covers 4 byte(s) for $100.00 \%$ of 0.01 seconds

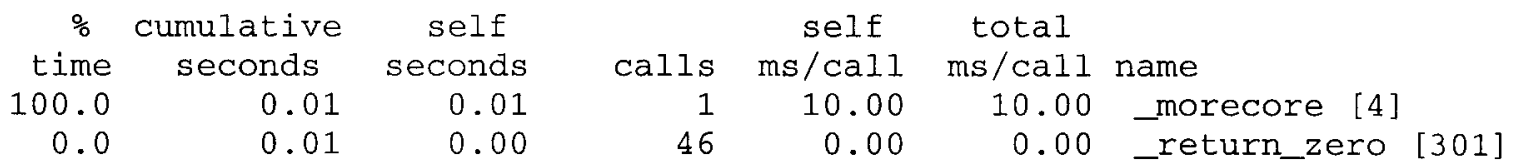




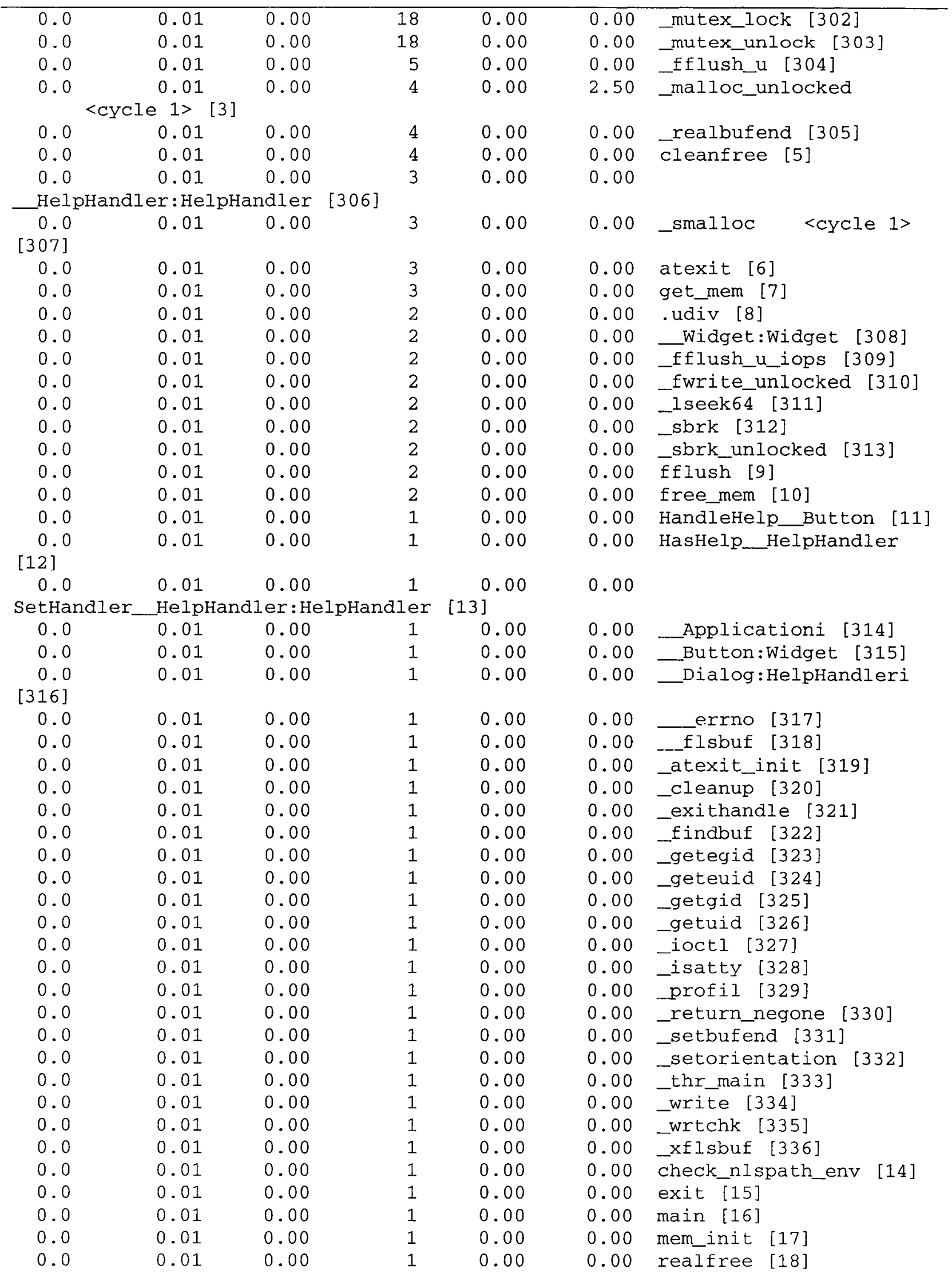


Index by function name

[8] .udiv [323] _getegid

[11] HandleHelp_Button [324] Geteuid

[12] HasHelp_HelpHand [325] _getgid

[13] SetHandler HelpH [326] -getuid

[314] Application [327]_ioct1

[306] _HelpHandler:H [328] _isatty

[315] _Button:Widget

[316] Dialog:HelpHan

[308] _Widget:Widget

[317] _- errno

[318] fflsbuf

[319] _atexit_init

[320] _cleanup

[321] _exithandle

[304]_fflush_u

[309] ffflush_u_iops

[322] _findbuf

[310] _fwrite_unlocked [311]_-1seek64

[3]_malloc_unlocked

[4] morecore

[302]_mutex_lock

[303]_mutex_unlock

[329] profil

[305] _realbufend

[330]_return_negone

[301] _return_zero

[312] _sbrk

[313] _sbrk_unlocked

[331] _setbufend
[332] _setorientation

[307] _smalloc

[333] _thr_main

[334] _write

[335]_wrtchk

[336]_xflsbuf

[6] atexit

[14] Check_nlspath_env

[5] cleanfree

[15] exit

[9] fflush

[10] free_mem

[7] get mem

[16] main

[17] mem_init

[18] realfree

[1] <cycle 1>

object modules

1: chain

The complete call graph for the sample code of Chain of Responsibility in Section 5.2.3 


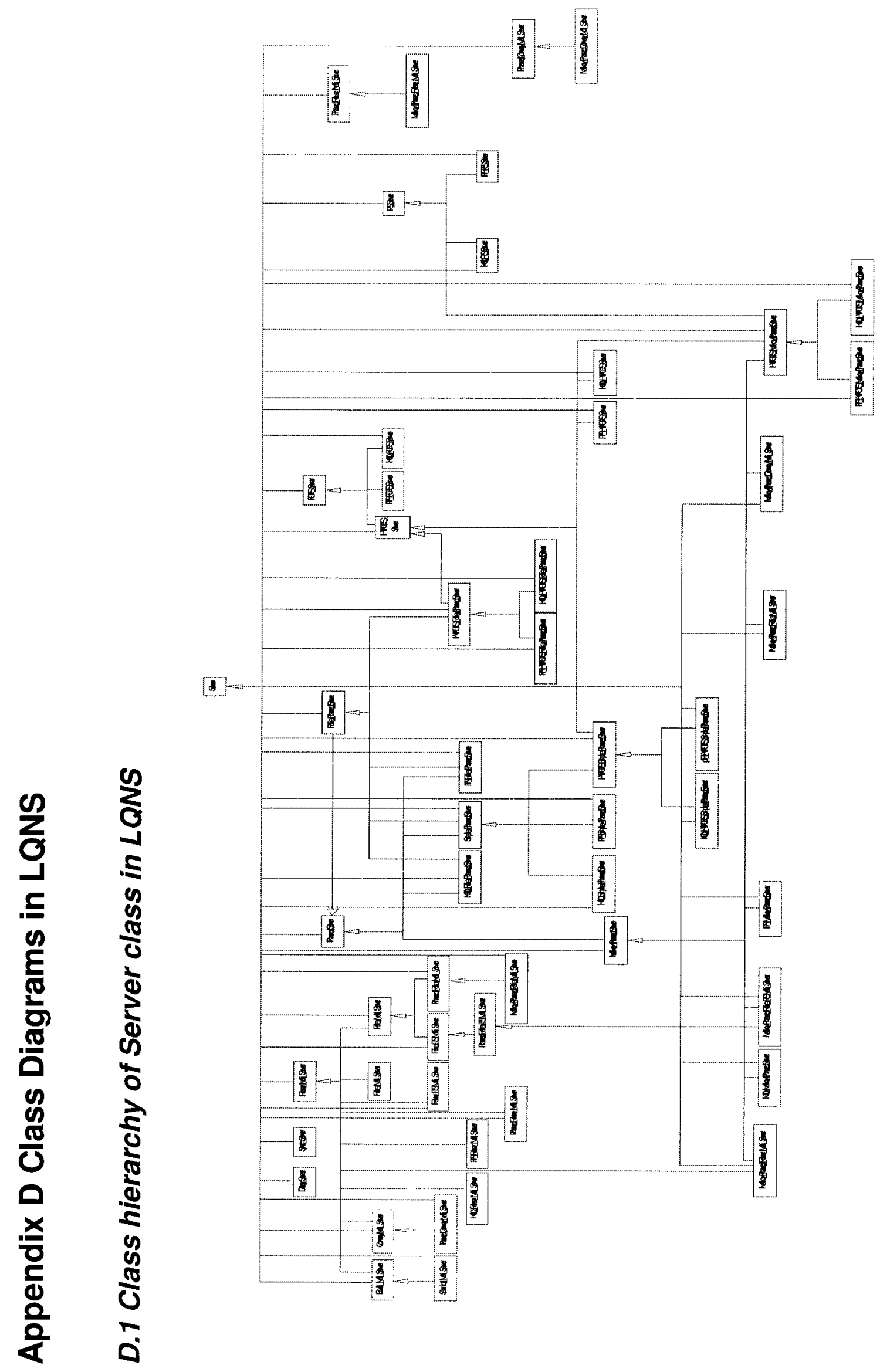




\section{D.2 Part of Class Diagram of LQNS}

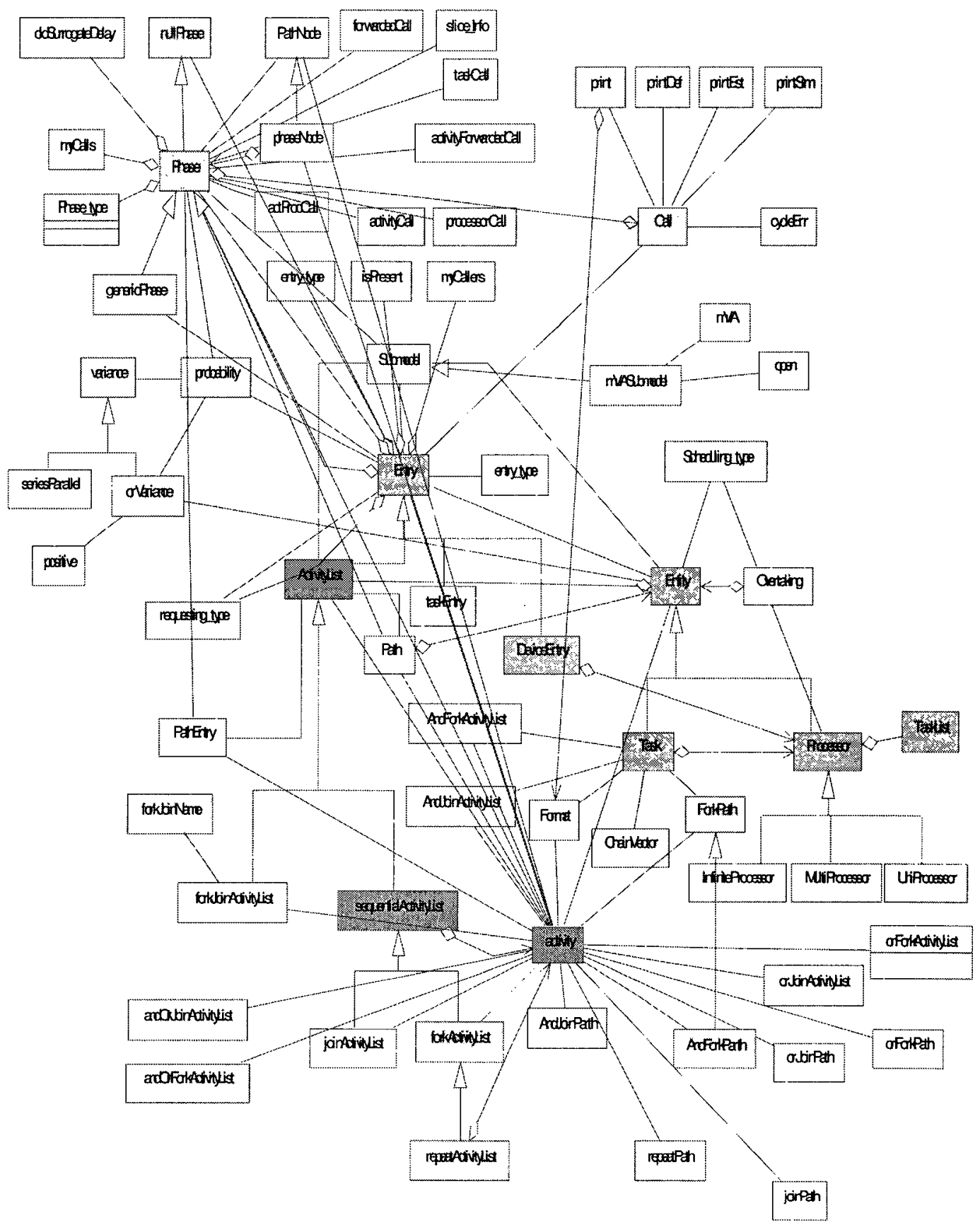

This class diagram is based on the database file "writepro.pl" generated by DPET. 